\title{
Intermolecular addition of carbon-centered radicals to ynamides - A regio- and stereoselective route to persubstituted a-iodo-enamides.
}

Nejib Dwadnia, Hugo Lingua, Dominique Mouysset, Liliane Mimoun, Didier Siri, Michèle P. Bertrand*, Laurence Feray*

Aix Marseille Univ, CNRS, ICR, Institut Chimie Radicalaire, UMR 7273, Equipes CMO et CT, Campus St Jérôme, Avenue Escadrille Normandie-Niemen, 13397 Marseille Cedex 20, France

Corresponding author's email address : laurence.feray@univ-amu.fr

\section{Supplementary Information - Table of content}

1.2. Cartesian coordinates and enthalpies (Hartree) of all calculated compounds at the B3LYP/6$31+G(d, p)$ level (B3LYP/Def2TZVP for iodine containing structures).

1.3. Crystal structure for $3 a b$. S34

1.4. Addition of $2 a$ to $1 d$. S35

1.5. NMR spectra for all new compounds S36 


\subsection{Computational details}

All the calculations were performed using the Gaussian 16 package. [Gaussian 16, Revision A.03, Frisch, M. J.; Trucks, G. W.; Schlegel, H. B.; Scuseria, G. E.; Robb, M. A.; Cheeseman, J. R.; Scalmani, G.; Barone, V.; Petersson, G. A.; Nakatsuji, H.; Li, X.; Caricato, M.; Marenich, A. V.; Bloino, J.; Janesko, B. G.; Gomperts, R.; Mennucci, B.; Hratchian, H. P.; Ortiz, J. V.; Izmaylov, A. F.; Sonnenberg, J. L.; Williams-Young, D.; Ding, F.; Lipparini, F.; Egidi, F.; Goings, J.; Peng, B.; Petrone, A.; Henderson, T.; Ranasinghe, D.; Zakrzewski, V. G.; Gao, J.; Rega, N.; Zheng, G.; Liang, W.; Hada, M.; Ehara, M.; Toyota, K.; Fukuda, R.; Hasegawa, J.; Ishida, M.; Nakajima, T.; Honda, Y.; Kitao, O.; Nakai, H.; Vreven, T.; Throssell, K.; Montgomery, J. A., Jr.; Peralta, J. E.; Ogliaro, F.; Bearpark, M. J.; Heyd, J. J.; Brothers, E. N.; Kudin, K. N.; Staroverov, V. N.; Keith, T. A.; Kobayashi, R.; Normand, J.; Raghavachari, K.; Rendell, A. P.; Burant, J. C.; Iyengar, S. S.; Tomasi, J.; Cossi, M.; Millam, J. M.; Klene, M.; Adamo, C.; Cammi, R.; Ochterski, J. W.; Martin, R. L.; Morokuma, K.; Farkas, O.; Foresman, J. B.; Fox, D. J. Gaussian, Inc., Wallingford CT, 2016.]. As regard to addition reactions, the geometry of all species was optimized at the B3LYP/6$31+G(d, p)$ level of theory. Vibrational frequencies were calculated to insure, that the obtained geometries were minima or transition states ( 0 or 1 imaginary frequency respectively) and to determine enthalpy values. Intrinsic reaction coordinate (IRC) calculations, at the same level of theory, were performed to insure that the transition states led to the expected reactants and products. Since the $6-31+G(d, p)$ basis set is not available for iodine atom, Def2TZVP basis set was selected to calculate reaction pathways involving iodine atom transfer.

\footnotetext{
1.2. Cartesian coordinates and enthalpies (Hartree) of all calculated compounds at the B3LYP/6-31+G(d,p) level (B3LYP/Def2TZVP for iodine containing structures).
}

\section{B3LYP/6-31+G(d,p) calculations}




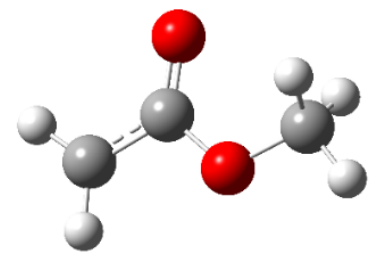

$\begin{array}{lr}\mathrm{C} & -1.78991 \\ \mathrm{H} & -1.82277 \\ \mathrm{H} & -2.70269 \\ \mathrm{C} & -0.52774 \\ \mathrm{O} & -0.41243 \\ \mathrm{O} & 0.53606 \\ \mathrm{C} & 1.83068 \\ \mathrm{H} & 1.95301 \\ \mathrm{H} & 2.54904 \\ \mathrm{H} & 1.95614\end{array}$

$$
\begin{array}{r}
-0.58567 \\
-1.66830 \\
-0.00419 \\
0.12238 \\
1.34275 \\
-0.72626 \\
-0.10061 \\
0.52518 \\
-0.91992 \\
0.51867
\end{array}
$$$$
0.00004
$$$$
-0.00025
$$$$
0.00028
$$$$
-0.00001
$$$$
-0.00004
$$$$
-0.00006
$$$$
0.00008
$$$$
0.88792
$$$$
0.00419
$$$$
-0.89198
$$

$\mathrm{H}=-267.661856$ a.u.

\section{1a-Me}

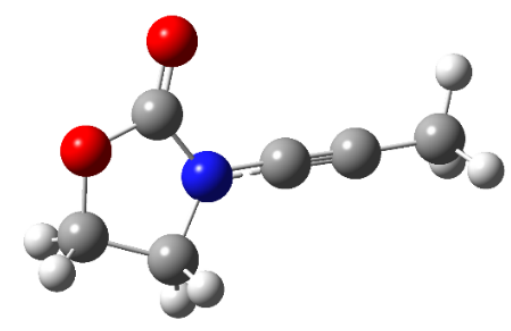

$\begin{array}{lr}\mathrm{C} & -0.00274 \\ \mathrm{O} & -0.01382 \\ \mathrm{C} & 1.26525 \\ \mathrm{C} & 1.40519 \\ \mathrm{H} & -0.79868 \\ \mathrm{H} & -0.19445 \\ \mathrm{H} & 1.81731 \\ \mathrm{H} & 1.44598 \\ \mathrm{~N} & 2.13563 \\ \mathrm{O} & 1.54943 \\ \mathrm{C} & 3.47084 \\ \mathrm{C} & 4.67164 \\ \mathrm{C} & 6.11729 \\ \mathrm{H} & 6.44813 \\ \mathrm{H} & 6.66335 \\ \mathrm{H} & 6.40813\end{array}$

$$
\begin{array}{r}
0.03265 \\
-0.11556 \\
-0.14950 \\
-0.40669 \\
-0.58908 \\
1.08390 \\
0.21421 \\
-1.45956 \\
-0.20608 \\
-0.13561 \\
-0.37405 \\
-0.51163 \\
-0.67707 \\
-1.64881 \\
0.10569 \\
-0.62006
\end{array}
$$$$
0.00960
$$$$
1.44376
$$$$
1.92641
$$$$
-0.42078
$$$$
-0.40215
$$$$
-0.22929
$$$$
-1.21976
$$$$
-0.72730
$$$$
0.83325
$$$$
3.09566
$$$$
0.95316
$$$$
1.02705
$$$$
1.15520
$$$$
0.77187
$$$$
0.61679
$$

2.20998

$\mathrm{H}=-437.883601$ a.u. 


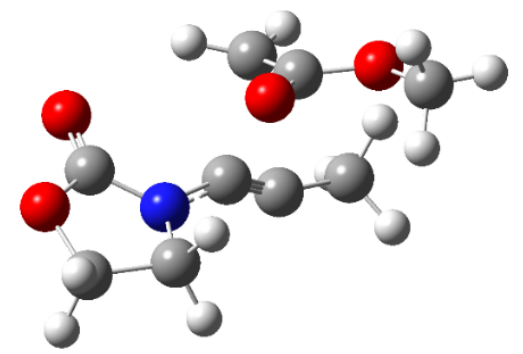

$\begin{array}{lrrr}\mathrm{C} & -2.87101 & -1.10079 & -1.14114 \\ \mathrm{O} & -3.10643 & -0.77804 & 0.24676 \\ \mathrm{C} & -2.21886 & 0.16131 & 0.67278 \\ \mathrm{C} & -1.45675 & -0.57554 & -1.44100 \\ \mathrm{H} & -2.96267 & -2.18144 & -1.25550 \\ \mathrm{H} & -3.63641 & -0.59807 & -1.74087 \\ \mathrm{H} & -1.38967 & -0.08150 & -2.41371 \\ \mathrm{H} & -0.69126 & -1.35267 & -1.36017 \\ \mathrm{~N} & -1.30532 & 0.39934 & -0.35183 \\ \mathrm{O} & -2.24744 & 0.67829 & 1.76337 \\ \mathrm{C} & -0.20549 & 1.19338 & -0.20462 \\ \mathrm{C} & 0.48391 & 2.15869 & -0.58015 \\ \mathrm{C} & 1.00897 & 0.20870 & 1.39598 \\ \mathrm{H} & 0.12693 & -0.03380 & 1.97485 \\ \mathrm{H} & 1.62871 & 1.02371 & 1.74848 \\ \mathrm{C} & 1.63506 & -0.85559 & 0.63476 \\ \mathrm{O} & 1.09229 & -1.90105 & 0.28564 \\ \mathrm{O} & 2.92208 & -0.55418 & 0.29028 \\ \mathrm{C} & 3.60619 & -1.54266 & -0.49514 \\ \mathrm{H} & 3.10546 & -1.68617 & -1.45686 \\ \mathrm{H} & 4.61288 & -1.15154 & -0.64387 \\ \mathrm{H} & 3.63998 & -2.49915 & 0.03265 \\ \mathrm{C} & 1.62291 & 3.06569 & -0.67417 \\ \mathrm{H} & 2.46635 & 2.71037 & -0.06640 \\ \mathrm{H} & 1.97075 & 3.14326 & -1.71041 \\ \mathrm{H} & 1.35258 & 4.07230 & -0.33754\end{array}$

$\mathrm{H}=-705.533992$ a.u.

Imaginary frequency $=i 542 \mathrm{~cm}^{-1}$

Addition to 1a-Me: vinyl radical at $\mathrm{C} \beta$

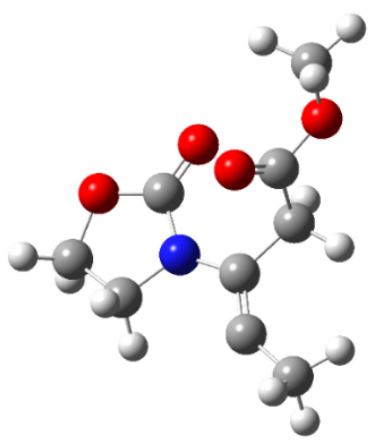




$\begin{array}{rrr}-3.24423 & -1.15477 & -0.36453 \\ -2.16511 & -2.04529 & -0.02402 \\ -1.07214 & -1.32942 & 0.38074 \\ -2.58436 & 0.22425 & -0.51581 \\ -3.70983 & -1.51742 & -1.28225 \\ -3.97609 & -1.17612 & 0.44960 \\ -3.16584 & 1.02357 & -0.04660 \\ -2.39599 & 0.50031 & -1.56133 \\ -1.32306 & 0.01572 & 0.18392 \\ -0.08214 & -1.85778 & 0.83805 \\ -0.40796 & 1.08665 & 0.34465 \\ -0.78134 & 2.32296 & 0.03861 \\ 0.98293 & 0.77000 & 0.86081 \\ 0.94991 & 0.18083 & 1.77951 \\ 1.49350 & 1.70915 & 1.09768 \\ 1.84122 & 0.02856 & -0.15491 \\ 1.59843 & -0.08037 & -1.33859 \\ 2.95150 & -0.45584 & 0.43388 \\ 3.83847 & -1.20717 & -0.41798 \\ 4.18914 & -0.58791 & -1.24714 \\ 4.66940 & -1.50457 & 0.22101 \\ 3.32186 & -2.08486 & -0.81281 \\ -0.26773 & 3.69857 & -0.01598 \\ 0.77004 & 3.75575 & 0.35176 \\ -0.26719 & 4.08610 & -1.04205 \\ -0.86806 & 4.38171 & 0.59706\end{array}$

$\mathrm{H}=-705.581252$ a.u.

1a-Me Addition at Ca: TS2

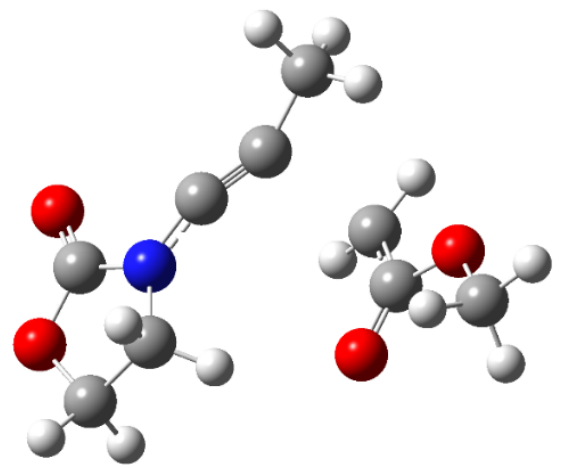

$\begin{array}{rrrr}\mathrm{C} & 2.67347 & -1.88958 & -0.16545 \\ \mathrm{O} & 3.55016 & -0.77614 & 0.12823 \\ \mathrm{C} & 2.84366 & 0.38449 & 0.17483 \\ \mathrm{C} & 1.42998 & -1.25872 & -0.80669 \\ \mathrm{H} & 3.20910 & -2.57098 & -0.82693 \\ \mathrm{H} & 2.43704 & -2.39459 & 0.77603 \\ \mathrm{H} & 0.49380 & -1.71444 & -0.47898 \\ \mathrm{H} & 1.48293 & -1.24689 & -1.90178 \\ \mathrm{~N} & 1.54045 & 0.11420 & -0.28319 \\ \mathrm{O} & 3.28174 & 1.44448 & 0.53839 \\ \mathrm{C} & 0.59088 & 1.04528 & -0.40771 \\ \mathrm{C} & -0.46316 & 1.68639 & -0.29648 \\ \mathrm{C} & -1.63077 & 0.64838 & 1.38770\end{array}$




$\begin{array}{rrrr}\mathrm{H} & -0.78261 & 0.46216 & 2.03240 \\ \mathrm{H} & -2.28008 & 1.48328 & 1.62193 \\ \mathrm{C} & -2.17128 & -0.48433 & 0.67189 \\ \mathrm{O} & -1.60338 & -1.56286 & 0.49817 \\ \mathrm{O} & -3.40307 & -0.22117 & 0.13930 \\ \mathrm{C} & -4.00180 & -1.28892 & -0.61043 \\ \mathrm{H} & -4.96971 & -0.90840 & -0.93748 \\ \mathrm{H} & -3.38240 & -1.55180 & -1.47254 \\ \mathrm{H} & -4.13053 & -2.17516 & 0.01649 \\ \mathrm{C} & -1.37362 & 2.76047 & -0.73736 \\ \mathrm{H} & -0.97112 & 3.28095 & -1.61178 \\ \mathrm{H} & -2.35495 & 2.34823 & -0.99270 \\ \mathrm{H} & -1.52234 & 3.49297 & 0.06317\end{array}$

$\mathrm{H}=-705.540020$ a.u.

Imaginary frequency $=i 399 \mathrm{~cm}^{-1}$

\section{Addition to 1a-Me: vinyl radical at $\mathrm{C} \alpha$}

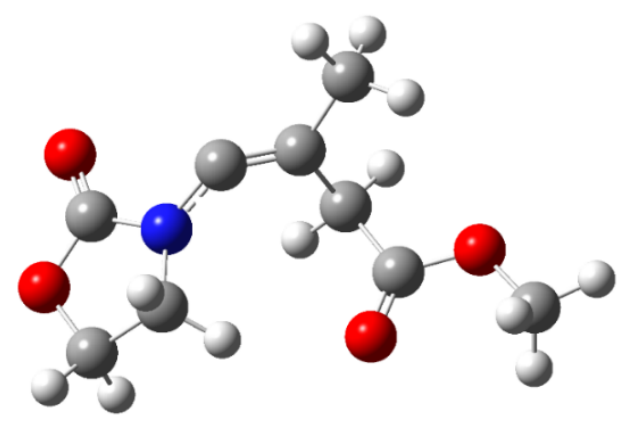

$\begin{array}{lr}\mathrm{C} & -2.72738 \\ \mathrm{O} & -3.54079 \\ \mathrm{C} & -2.80320 \\ \mathrm{C} & -1.55270 \\ \mathrm{H} & -3.34469 \\ \mathrm{H} & -2.39736 \\ \mathrm{H} & -0.59900 \\ \mathrm{H} & -1.73796 \\ \mathrm{~N} & -1.55115 \\ \mathrm{O} & -3.19052 \\ \mathrm{C} & -0.66860 \\ \mathrm{C} & 0.56046 \\ \mathrm{C} & 1.21465 \\ \mathrm{H} & 0.44251 \\ \mathrm{H} & 1.80087 \\ \mathrm{C} & 2.11142 \\ \mathrm{O} & 1.71679 \\ \mathrm{O} & 3.41126 \\ \mathrm{C} & 4.33845 \\ \mathrm{H} & 5.32016 \\ \mathrm{H} & 4.09951 \\ \mathrm{H} & 4.29681 \\ \mathrm{C} & 1.36889 \\ \mathrm{H} & 0.81937 \\ \mathrm{H} & 2.32238\end{array}$

$$
\begin{array}{r}
-1.85644 \\
-0.75816 \\
0.38900 \\
-1.19063 \\
-2.48291 \\
-2.43367 \\
-1.69755 \\
-1.07782 \\
0.12926 \\
1.42974 \\
1.12384 \\
1.41376 \\
0.62960 \\
0.15582 \\
1.31034 \\
-0.47461 \\
-1.55709 \\
-0.11417 \\
-1.09186 \\
-0.62570 \\
-1.32917 \\
-2.00534 \\
2.52849 \\
3.00767 \\
2.15463
\end{array}
$$$$
0.19415
$$$$
-0.27318
$$$$
-0.31228
$$$$
0.92108
$$$$
0.83915
$$$$
-0.67567
$$$$
0.76294
$$$$
1.99655
$$$$
0.26877
$$$$
-0.77919
$$$$
0.59712
$$$$
0.19178
$$$$
-0.95272
$$$$
-1.56304
$$$$
-1.57661
$$$$
-0.42334
$$$$
-0.02969
$$$$
-0.41418
$$$$
0.10266
$$$$
0.02802
$$$$
1.14188
$$$$
-0.49478
$$$$
0.80743
$$

1.62024

1.20010 


\section{$\mathrm{H}=-705.584824$ a.u.}

\section{1c-Me}

\begin{tabular}{|c|c|}
\hline $\mathrm{N}$ & -0.26917 \\
\hline C & -0.21183 \\
\hline $\mathrm{C}$ & -0.18047 \\
\hline $\mathrm{C}$ & -0.14374 \\
\hline S & 1.20393 \\
\hline O & 0.89066 \\
\hline 0 & 1.73309 \\
\hline $\mathrm{C}$ & 2.31137 \\
\hline $\mathrm{C}$ & 3.26067 \\
\hline $\mathrm{C}$ & 2.22047 \\
\hline $\mathrm{C}$ & 4.13598 \\
\hline $\mathrm{H}$ & 3.31082 \\
\hline $\mathrm{C}$ & 3.10016 \\
\hline $\mathrm{H}$ & 1.48498 \\
\hline $\mathrm{C}$ & 4.05424 \\
\hline $\mathrm{H}$ & 4.88160 \\
\hline $\mathrm{H}$ & 3.04531 \\
\hline $\mathrm{H}$ & 4.73799 \\
\hline C & -1.54165 \\
\hline $\mathrm{H}$ & -1.80442 \\
\hline $\mathrm{H}$ & -1.33417 \\
\hline C & -2.6646 \\
\hline C & -2.66187 \\
\hline C & -3.73080 \\
\hline C & -3.70360 \\
\hline $\mathrm{H}$ & -1.83823 \\
\hline C & -4.77910 \\
\hline $\mathrm{H}$ & -3.74152 \\
\hline C & -4.76671 \\
\hline $\mathrm{H}$ & -3.6912 \\
\hline $\mathrm{H}$ & -5.6011 \\
\hline $\mathrm{H}$ & -5.5800 \\
\hline $\mathrm{H}$ & 0.37021 \\
\hline $\mathrm{H}$ & -1.1623 \\
\hline $\mathrm{H}$ & 0.3625 \\
\hline
\end{tabular}

$\begin{array}{rr}0.24043 & -0.72168 \\ 1.43980 & -0.08939 \\ 2.49604 & 0.50511 \\ 3.76525 & 1.22731 \\ -0.28145 & -1.49249 \\ -1.61447 & -2.02317 \\ 0.76607 & -2.37233 \\ -0.44260 & -0.08900 \\ 0.55363 & 0.14194 \\ -1.57825 & 0.71948 \\ 0.40876 & 1.22106 \\ 1.41309 & -0.51690 \\ -1.70915 & 1.79410 \\ -2.34462 & 0.50129 \\ -0.71685 & 2.04498 \\ 1.17400 & 1.41385 \\ -2.58721 & 2.43022 \\ -0.82513 & 2.88178 \\ -0.11278 & -1.42178 \\ 0.68481 & -2.12596 \\ -1.02327 & -1.98707 \\ -0.34372 & -0.43573 \\ -1.47748 & 0.38985 \\ 0.55794 & -0.34458 \\ -1.70263 & 1.28962 \\ -2.18353 & 0.32413 \\ 0.33354 & 0.55363 \\ 1.44019 & -0.97948 \\ -0.79634 & 1.37341 \\ -2.58649 & 1.92109 \\ 1.04125 & 0.61188 \\ -0.97322 & 2.07136 \\ 4.54308 & 0.65112 \\ 4.11854 & 1.42405 \\ 3.66491 & \end{array}$




\section{Addition to 1c-Me: TS1}

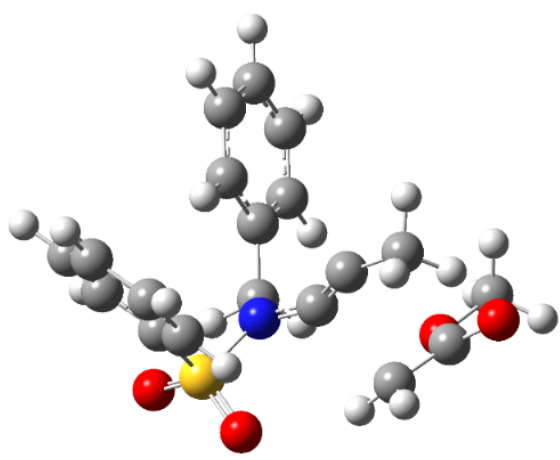

\begin{tabular}{|c|c|c|c|}
\hline $\mathrm{N}$ & -0.11357 & -0.24110 & -0.64256 \\
\hline $\mathrm{C}$ & 0.34690 & -0.93248 & 0.44343 \\
\hline $\mathrm{C}$ & 0.33749 & -1.13768 & 1.67518 \\
\hline $\mathrm{C}$ & 0.69965 & -1.78695 & 2.93151 \\
\hline S & -1.51748 & -0.85938 & -1.43215 \\
\hline O & -1.63566 & -0.08967 & -2.67522 \\
\hline O & -1.43639 & -2.32185 & -1.43731 \\
\hline $\mathrm{C}$ & -2.86400 & -0.38930 & -0.34100 \\
\hline C & -3.31127 & -1.29680 & 0.62142 \\
\hline C & -3.44756 & 0.87191 & -0.49190 \\
\hline $\mathrm{C}$ & -4.36161 & -0.92109 & 1.46227 \\
\hline $\mathrm{H}$ & -2.85529 & -2.27752 & 0.69289 \\
\hline C & -4.49710 & 1.23229 & 0.35424 \\
\hline $\mathrm{H}$ & -3.09725 & 1.54408 & -1.26764 \\
\hline C & -4.95046 & 0.33946 & 1.33122 \\
\hline $\mathrm{H}$ & -4.72279 & -1.61709 & 2.21319 \\
\hline $\mathrm{H}$ & -4.96562 & 2.20547 & 0.24403 \\
\hline $\mathrm{H}$ & -5.76952 & 0.62426 & 1.98491 \\
\hline $\mathrm{C}$ & 0.77452 & 0.71551 & -1.33572 \\
\hline $\mathrm{H}$ & 1.70108 & 0.20205 & -1.61543 \\
\hline $\mathrm{H}$ & 0.26224 & 1.00003 & -2.25818 \\
\hline $\mathrm{C}$ & 1.09536 & 1.94615 & -0.50700 \\
\hline $\mathrm{C}$ & 0.18466 & 2.48594 & 0.40927 \\
\hline $\mathrm{C}$ & 2.32204 & 2.59409 & -0.70336 \\
\hline $\mathrm{C}$ & 0.49184 & 3.65535 & 1.10931 \\
\hline $\mathrm{H}$ & -0.76037 & 1.98140 & 0.58541 \\
\hline C & 2.62805 & 3.76789 & -0.01039 \\
\hline $\mathrm{H}$ & 3.04292 & 2.17683 & -1.40234 \\
\hline $\mathrm{C}$ & 1.71260 & 4.30251 & 0.89992 \\
\hline $\mathrm{H}$ & -0.22295 & 4.05922 & 1.82086 \\
\hline $\mathrm{H}$ & 3.58318 & 4.25839 & -0.17556 \\
\hline $\mathrm{H}$ & 1.95076 & 5.21115 & 1.44519 \\
\hline $\mathrm{H}$ & 0.83061 & -1.04966 & 3.73141 \\
\hline $\mathrm{H}$ & -0.08590 & -2.48409 & 3.24500 \\
\hline $\mathrm{H}$ & 1.64048 & -2.34145 & 2.81631 \\
\hline $\mathrm{C}$ & 1.75546 & -2.47519 & -0.37614 \\
\hline $\mathrm{H}$ & 1.54804 & -2.25325 & -1.41564 \\
\hline $\mathrm{H}$ & 1.26373 & -3.33882 & 0.05094 \\
\hline $\mathrm{C}$ & 3.05787 & -2.13871 & 0.16810 \\
\hline O & 3.49949 & -2.52196 & 1.24733 \\
\hline O & 3.75987 & -1.29202 & -0.64402 \\
\hline $\mathrm{C}$ & 5.04760 & -0.88640 & -0.15332 \\
\hline $\mathrm{H}$ & 5.47107 & -0.25307 & -0.93327 \\
\hline
\end{tabular}




$\begin{array}{llll}\mathrm{H} & 4.94444 & -0.32803 & 0.78097 \\ \mathrm{H} & 5.68513 & -1.75696 & 0.02085\end{array}$

$H=-1489.391420$ a.u.

Imaginary frequency $=i 541 \mathrm{~cm}^{-1}$

\section{Addition to 1c-Me: vinyl radical at $\mathrm{C} \beta$}

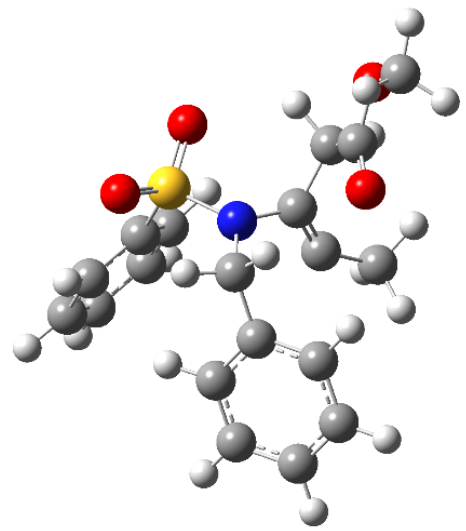

$\begin{array}{lrrr}\mathrm{N} & 0.47032 & -0.43096 & -0.50626 \\ \mathrm{C} & 1.05651 & -0.30123 & 0.81005 \\ \mathrm{C} & 0.49244 & 0.47554 & 1.71814 \\ \mathrm{C} & 0.69704 & 1.01833 & 3.06609 \\ \mathrm{~S} & -0.48333 & -1.77906 & -0.82739 \\ \mathrm{O} & -0.64387 & -1.84277 & -2.28582 \\ \mathrm{O} & 0.11195 & -2.88730 & -0.06798 \\ \mathrm{C} & -2.13006 & -1.51832 & -0.13575 \\ \mathrm{C} & -2.34334 & -1.72172 & 1.23098 \\ \mathrm{C} & -3.17379 & -1.14450 & -0.98565 \\ \mathrm{C} & -3.62179 & -1.52111 & 1.75448 \\ \mathrm{H} & -1.52723 & -2.05142 & 1.86329 \\ \mathrm{C} & -4.45023 & -0.95387 & -0.45100 \\ \mathrm{H} & -2.98636 & -1.03223 & -2.04798 \\ \mathrm{C} & -4.67308 & -1.13511 & 0.91688 \\ \mathrm{H} & -3.79915 & -1.67923 & 2.81411 \\ \mathrm{H} & -5.27031 & -0.67410 & -1.10564 \\ \mathrm{H} & -5.66745 & -0.98828 & 1.32818 \\ \mathrm{C} & 0.49675 & 0.72673 & -1.44282 \\ \mathrm{H} & 1.54710 & 0.89894 & -1.68986 \\ \mathrm{H} & -0.00818 & 0.39664 & -2.35244 \\ \mathrm{C} & -0.11060 & 2.01398 & -0.91884 \\ \mathrm{C} & -1.49754 & 2.22512 & -0.91401 \\ \mathrm{C} & 0.72723 & 3.04406 & -0.46675 \\ \mathrm{C} & -2.03524 & 3.42915 & -0.45548 \\ \mathrm{H} & -2.16206 & 1.44363 & -1.26990 \\ \mathrm{C} & 0.19284 & 4.25306 & -0.01210 \\ \mathrm{H} & -1.80283 & 2.88955 & -0.47028 \\ \mathrm{C} & -3.11178 & 4.44778 & -0.00243 \\ \mathrm{H} & 0.85680 & 3.57586 & -0.45919 \\ \mathrm{H} & & 5.04231 & 0.32989\end{array}$




$\begin{array}{rrrr}\mathrm{H} & -1.60847 & 5.38752 & 0.34786 \\ \mathrm{H} & 0.75396 & 2.11282 & 3.03586 \\ \mathrm{H} & -0.13131 & 0.74983 & 3.73209 \\ \mathrm{H} & 1.62968 & 0.64748 & 3.52183 \\ \mathrm{C} & 2.35135 & -1.06152 & 1.03882 \\ \mathrm{H} & 2.21308 & -2.11431 & 0.77595 \\ \mathrm{H} & 2.63328 & -1.03699 & 2.09844 \\ \mathrm{C} & 3.52702 & -0.50769 & 0.24977 \\ \mathrm{O} & 3.57671 & 0.57741 & -0.29601 \\ \mathrm{O} & 4.55389 & -1.38236 & 0.25200 \\ \mathrm{C} & 5.74615 & -0.96510 & -0.44358 \\ \mathrm{H} & 6.44979 & -1.78885 & -0.32888 \\ \mathrm{H} & 5.52623 & -0.78924 & -1.49901 \\ \mathrm{H} & 6.14573 & -0.04967 & -0.00062\end{array}$

$\mathrm{H}=-1489.433566$ a.u.

Addition to 1c-Me:TS2

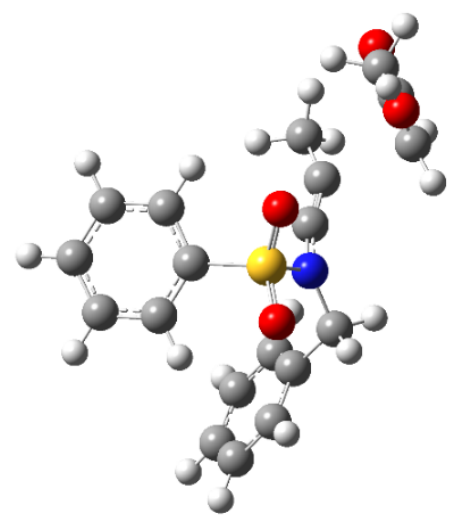

$\begin{array}{rrrr}\text { N } & -0.11420 & -0.73042 & -0.64707 \\ \mathrm{C} & -0.83388 & -0.85080 & 0.47336 \\ \mathrm{C} & -1.70609 & -1.07041 & 1.33061 \\ \mathrm{C} & -2.11889 & -1.01453 & 2.74980 \\ \mathrm{~S} & 0.00675 & 0.85260 & -1.37851 \\ \mathrm{O} & 0.82441 & 0.63527 & -2.57529 \\ \mathrm{O} & -1.35223 & 1.38174 & -1.44407 \\ \mathrm{C} & 0.92761 & 1.85677 & -0.20947 \\ \mathrm{C} & 0.22531 & 2.53927 & 0.78807 \\ \mathrm{C} & 2.31725 & 1.93974 & -0.32301 \\ \mathrm{C} & 0.94210 & 3.31761 & 1.69888 \\ \mathrm{H} & -0.85578 & 2.47369 & 0.83296 \\ \mathrm{C} & 3.01854 & 2.72546 & 0.59300 \\ \mathrm{H} & 2.83226 & 1.40871 & -1.11496 \\ \mathrm{C} & 2.33390 & 3.40964 & 1.60221 \\ \mathrm{H} & 0.41116 & 3.85743 & 2.47691 \\ \mathrm{H} & 4.09830 & 2.80536 & 0.51420 \\ \mathrm{H} & 2.88554 & 4.02042 & 2.31072 \\ \mathrm{C} & 0.78895 & -1.82451 & -1.10471 \\ \mathrm{H} & 0.24486 & -2.74613 & -0.87899 \\ \mathrm{H} & 0.86819 & -1.73528 & -2.18837 \\ \mathrm{C} & 2.16563 & -1.84078 & -0.47200 \\ \mathrm{C} & 2.33253 & -2.13622 & 0.88959 \\ \mathrm{C} & 3.30059 & -1.59373 & -1.25555\end{array}$




$\begin{array}{lrrr}\mathrm{C} & 3.60728 & -2.17001 & 1.45693 \\ \mathrm{H} & 1.46188 & -2.34080 & 1.50677 \\ \mathrm{C} & 4.57920 & -1.63621 & -0.69124 \\ \mathrm{H} & 3.18098 & -1.36572 & -2.31141 \\ \mathrm{C} & 4.73472 & -1.92098 & 0.66719 \\ \mathrm{H} & 3.72230 & -2.40044 & 2.51219 \\ \mathrm{H} & 5.45000 & -1.44843 & -1.31298 \\ \mathrm{H} & 5.72694 & -1.95593 & 1.10773 \\ \mathrm{H} & -3.12300 & -0.58709 & 2.83549 \\ \mathrm{H} & -2.15787 & -2.02476 & 3.17284 \\ \mathrm{H} & -1.41613 & -0.42074 & 3.34264 \\ \mathrm{C} & -3.53428 & -2.03738 & 0.38514 \\ \mathrm{H} & -3.68636 & -2.71190 & 1.21855 \\ \mathrm{H} & -3.10935 & -2.43733 & -0.52688 \\ \mathrm{C} & -4.42156 & -0.89482 & 0.29664 \\ \mathrm{O} & -5.04724 & -0.40652 & 1.23675 \\ \mathrm{O} & -4.45791 & -0.37214 & -0.95945 \\ \mathrm{C} & -5.22048 & 0.83219 & -1.11888 \\ \mathrm{H} & -4.81316 & 1.62939 & -0.49137 \\ \mathrm{H} & -5.12887 & 1.09646 & -2.17229 \\ \mathrm{H} & -6.26749 & 0.66561 & -0.85091 \\ & & & \end{array}$

$H=-1489.395107$ a.u.

Imaginary frequency $=i 408 \mathrm{~cm}^{-1}$

Addition to 1c-Me: vinyl radical at $\mathrm{C} \alpha$

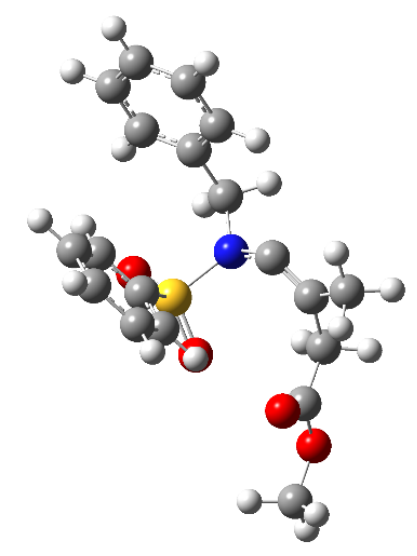

$\begin{array}{rrrr}\mathrm{N} & -0.16387 & 1.25020 & -0.39225 \\ \mathrm{C} & 0.40334 & 1.32795 & 0.84996 \\ \mathrm{C} & 1.64344 & 1.44138 & 1.32481 \\ \mathrm{C} & 1.91416 & 1.35264 & 2.80790 \\ \mathrm{~S} & 0.13825 & -0.14119 & -1.40753 \\ \mathrm{O} & -0.78529 & 0.00400 & -2.53904 \\ \mathrm{O} & 1.58757 & -0.19547 & -1.61463 \\ \mathrm{C} & -0.32844 & -1.57834 & -0.43975 \\ \mathrm{C} & 0.62216 & -2.16283 & 0.40269 \\ \mathrm{C} & -1.62796 & -2.07825 & -0.55522 \\ \mathrm{C} & 0.24605 & -3.27984 & 1.15224 \\ \mathrm{H} & 1.62733 & -1.75956 & 0.46577 \\ \mathrm{C} & -1.98309 & -3.19813 & 0.19794 \\ \mathrm{H} & -2.33743 & -1.60429 & -1.22366\end{array}$




$\begin{array}{rrrr}\mathrm{C} & -1.04922 & -3.79569 & 1.05042 \\ \mathrm{H} & 0.97129 & -3.74758 & 1.81098 \\ \mathrm{H} & -2.98672 & -3.60370 & 0.11514 \\ \mathrm{H} & -1.33120 & -4.66757 & 1.63362 \\ \mathrm{C} & -1.36351 & 2.07975 & -0.69427 \\ \mathrm{H} & -1.14543 & 3.05054 & -0.23977 \\ \mathrm{H} & -1.40187 & 2.21408 & -1.77546 \\ \mathrm{C} & -2.68793 & 1.54092 & -0.18604 \\ \mathrm{C} & -2.96185 & 1.47273 & 1.18827 \\ \mathrm{C} & -3.67499 & 1.13474 & -1.09392 \\ \mathrm{C} & -4.19308 & 0.99805 & 1.64376 \\ \mathrm{H} & -2.20362 & 1.78549 & 1.90073 \\ \mathrm{C} & -4.91220 & 0.66715 & -0.64002 \\ \mathrm{H} & -3.46918 & 1.17805 & -2.15979 \\ \mathrm{C} & -5.17298 & 0.59535 & 0.73028 \\ \mathrm{H} & -4.39079 & 0.94885 & 2.71081 \\ \mathrm{H} & -5.66874 & 0.36001 & -1.35670 \\ \mathrm{H} & -6.13341 & 0.23259 & 1.08536 \\ \mathrm{H} & 2.60184 & 0.52670 & 3.02048 \\ \mathrm{H} & 2.38828 & 2.27580 & 3.16825 \\ \mathrm{H} & 0.99134 & 1.19481 & 3.37049 \\ \mathrm{C} & 2.83858 & 1.71632 & 0.43033 \\ \mathrm{H} & 3.42387 & 2.55395 & 0.83487 \\ \mathrm{H} & 2.52650 & 1.99779 & -0.57810 \\ \mathrm{C} & 3.78448 & 0.53215 & 0.29420 \\ \mathrm{O} & 3.68817 & -0.53449 & 0.86955 \\ \mathrm{O} & 4.78604 & 0.82624 & -0.55817 \\ \mathrm{C} & 5.73058 & -0.23145 & -0.81598 \\ \mathrm{H} & 5.22067 & -1.09136 & -1.25609 \\ \mathrm{H} & 6.45033 & 0.18947 & -1.51739 \\ \mathrm{H} & 6.22488 & -0.53636 & 0.10955 \\ & & & \end{array}$

$\mathrm{H}=-1489.445840$ a.u.

\section{B3LYP/Def2TZVP calculations}

\section{Methyl $\alpha$-iodoacetate}

$\begin{array}{rrrr}\mathrm{C} & -1.53928 & 0.58590 & 0.11193 \\ \mathrm{O} & -2.01540 & 1.31677 & -0.71755 \\ \mathrm{O} & -2.08750 & -0.57596 & 0.50294 \\ \mathrm{C} & -3.29726 & -0.95871 & -0.17383 \\ \mathrm{H} & -3.58396 & -1.91211 & 0.26043 \\ \mathrm{H} & -4.07484 & -0.21304 & -0.01328 \\ \mathrm{H} & -3.11544 & -1.06213 & -1.24284 \\ \mathrm{C} & -0.26255 & 0.86612 & 0.85588\end{array}$




$\begin{array}{rrrr}\mathrm{I} & 1.40582 & -0.15316 & -0.08934 \\ \mathrm{H} & -0.02800 & 1.92231 & 0.80857 \\ \mathrm{H} & -0.28849 & 0.49633 & 1.87488\end{array}$

$\mathrm{H}=-565.609678$ a.u.

\section{$\alpha$-Methoxycarbonyl radical}

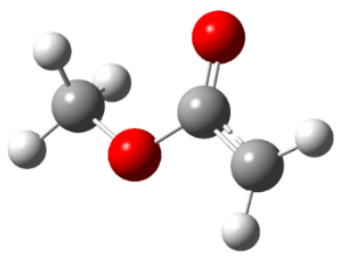

$\begin{array}{rrrr}\mathrm{C} & 0.52569 & 0.12396 & 0.00003 \\ \mathrm{O} & 0.41226 & 1.33449 & 0.00001 \\ \mathrm{O} & -0.53418 & -0.72323 & 0.00007 \\ \mathrm{C} & -1.82469 & -0.09998 & -0.00005 \\ \mathrm{H} & -2.54388 & -0.91463 & -0.00038 \\ \mathrm{H} & -1.95097 & 0.52143 & 0.88662 \\ \mathrm{H} & -1.95060 & 0.52185 & -0.88646 \\ \mathrm{C} & 1.78382 & -0.58282 & -0.00002 \\ \mathrm{H} & 2.69409 & -0.00373 & -0.00008 \\ \mathrm{H} & 1.81777 & -1.66195 & -0.00010\end{array}$

$\mathrm{H}=-267.756480$ a.u.

$\mathbf{A}^{\cdot}$

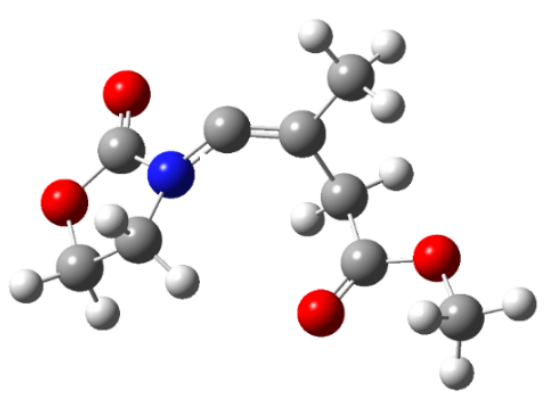

$\begin{array}{ll}\mathrm{C} & -2.74182 \\ \mathrm{O} & -3.53533 \\ \mathrm{C} & -2.79339 \\ \mathrm{C} & -1.55997 \\ \mathrm{H} & -3.36399 \\ \mathrm{H} & -2.42003 \\ \mathrm{H} & -0.61582 \\ \mathrm{H} & -1.73292 \\ \mathrm{~N} & -1.55358 \\ \mathrm{O} & -3.16745 \\ \mathrm{C} & -0.65729 \\ \mathrm{C} & 0.56093\end{array}$

$$
\begin{array}{r}
-1.84044 \\
-0.73945 \\
0.40019 \\
-1.19019 \\
-2.45113 \\
-2.43243 \\
-1.70395 \\
-1.09087 \\
0.13181 \\
1.43552 \\
1.10819 \\
1.39525
\end{array}
$$

0.19867

$-0.27965$

$-0.30885$

0.91891

0.84886

$-0.66019$

0.74938

1.99418

0.28384

$-0.77294$

0.60022

0.18987 


$\begin{array}{lrrr}\mathrm{C} & 1.20512 & 0.61494 & -0.95637 \\ \mathrm{H} & 0.43251 & 0.13868 & -1.55753 \\ \mathrm{H} & 1.78054 & 1.29627 & -1.58384 \\ \mathrm{C} & 2.10857 & -0.48227 & -0.43333 \\ \mathrm{O} & 1.72678 & -1.56766 & -0.06692 \\ \mathrm{O} & 3.39788 & -0.10168 & -0.39807 \\ \mathrm{C} & 4.32928 & -1.06859 & 0.11878 \\ \mathrm{H} & 5.30262 & -0.58842 & 0.07318 \\ \mathrm{H} & 4.07680 & -1.32921 & 1.14585 \\ \mathrm{H} & 4.31739 & -1.97065 & -0.49149 \\ \mathrm{C} & 1.37394 & 2.50150 & 0.80220 \\ \mathrm{H} & 0.83518 & 2.97406 & 1.62123 \\ \mathrm{H} & 2.32849 & 2.12526 & 1.18013 \\ \mathrm{H} & 1.60702 & 3.26437 & 0.05349\end{array}$

$\mathrm{H}=-705.827011$ a.u.

\section{$\mathbf{A}^{\bullet} \rightarrow \mathbf{B}^{\bullet}: \mathbf{T S}_{\text {inv }}$}

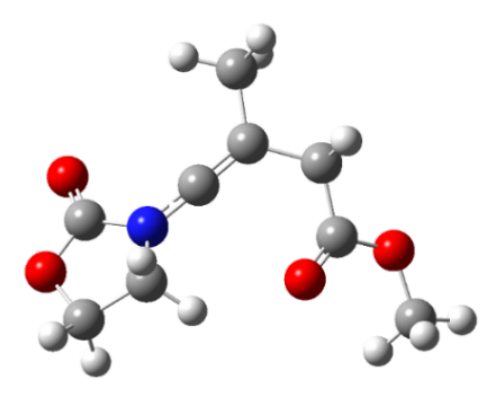

$\begin{array}{lr}\mathrm{C} & -2.75623 \\ \mathrm{O} & -3.46888 \\ \mathrm{C} & -2.66874 \\ \mathrm{C} & -1.55162 \\ \mathrm{H} & -3.43350 \\ \mathrm{H} & -2.45698 \\ \mathrm{H} & -0.62993 \\ \mathrm{H} & -1.72888 \\ \mathrm{~N} & -1.46620 \\ \mathrm{O} & -2.96656 \\ \mathrm{C} & -0.44831 \\ \mathrm{C} & 0.53660 \\ \mathrm{C} & 2.01167 \\ \mathrm{H} & 2.58463 \\ \mathrm{H} & 2.41173 \\ \mathrm{C} & 2.33376 \\ \mathrm{O} & 1.65924 \\ \mathrm{O} & 3.52142 \\ \mathrm{C} & 3.96927 \\ \mathrm{H} & 4.93367 \\ \mathrm{H} & 4.07044 \\ \mathrm{H} & 3.26540 \\ \mathrm{C} & 0.31849 \\ \mathrm{H} & -0.74092 \\ \mathrm{H} & 0.73703 \\ \mathrm{H} & 0.81965\end{array}$

$\begin{array}{rr}-1.97767 & 0.27264 \\ -0.90178 & -0.36818 \\ 0.18703 & -0.50305 \\ -1.31824 & 0.94296 \\ -2.46009 & 0.97391 \\ -2.69322 & -0.49549 \\ -1.88453 & 0.84574 \\ -1.09822 & 1.99891 \\ -0.06304 & 0.17686 \\ 1.17501 & -1.10616 \\ 0.78546 & 0.22124 \\ 1.63623 & 0.27258 \\ 1.24570 & 0.33730 \\ 1.83222 & -0.38367 \\ 1.51899 & 1.32353 \\ -0.21348 & 0.13335 \\ -1.15006 & 0.48631 \\ -0.36030 & -0.48513 \\ -1.71082 & -0.68459 \\ -1.62797 & -1.17790 \\ -2.22520 & 0.27054 \\ -2.25735 & -1.31120 \\ 3.14115 & 0.28282 \\ 3.38227 & 0.23101 \\ 3.58846 & 1.19039 \\ 3.61075 & -0.57000\end{array}$

$H=-705.812689$ a.u. 
Imaginary frequency $=i 191 \mathrm{~cm}^{-1}$

$\mathbf{B}^{\bullet}$

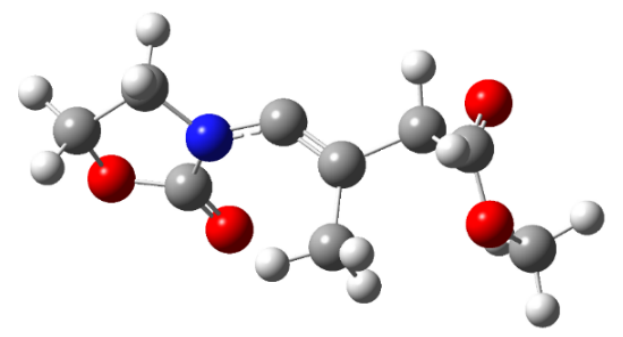

$\begin{array}{lr}\mathrm{C} & 4.10097 \\ \mathrm{O} & 3.37249 \\ \mathrm{C} & 2.04069 \\ \mathrm{C} & 3.05018 \\ \mathrm{H} & 4.87860 \\ \mathrm{H} & 4.55998 \\ \mathrm{H} & 3.19101 \\ \mathrm{H} & 3.01625 \\ \mathrm{~N} & 1.82300 \\ \mathrm{O} & 1.22413 \\ \mathrm{C} & 0.59534 \\ \mathrm{C} & -0.48304 \\ \mathrm{C} & -1.78718 \\ \mathrm{H} & -1.59075 \\ \mathrm{H} & -2.32585 \\ \mathrm{C} & -2.69346 \\ \mathrm{O} & -2.94454 \\ \mathrm{O} & -3.19007 \\ \mathrm{C} & -4.01644 \\ \mathrm{H} & -4.31510 \\ \mathrm{H} & -3.45269 \\ \mathrm{H} & -4.88970 \\ \mathrm{C} & -0.48173 \\ \mathrm{H} & 0.51909 \\ \mathrm{H} & -1.12484 \\ \mathrm{H} & -0.87518\end{array}$

$$
\begin{array}{r}
-0.03153 \\
1.20739 \\
0.97930 \\
-1.10978 \\
0.01838 \\
-0.14585 \\
-2.00163 \\
-1.40362 \\
-0.40751 \\
1.83913 \\
-0.98086 \\
-0.96870 \\
-1.52110 \\
-2.21490 \\
-2.04429 \\
-0.43829 \\
-0.29137 \\
0.35051 \\
1.44259 \\
1.96368 \\
2.10538 \\
1.06936 \\
-0.44137 \\
-0.16088 \\
0.43724 \\
-1.19706
\end{array}
$$$$
-0.19909
$$$$
-0.21851
$$$$
-0.06303
$$$$
-0.47396
$$$$
-0.95772
$$$$
0.78474
$$$$
0.13510
$$$$
-1.52605
$$$$
-0.10394
$$$$
0.07673
$$$$
-0.18915
$$$$
0.57254
$$$$
0.02592
$$$$
-0.78726
$$$$
0.82083
$$$$
-0.53581
$$$$
-1.70317
$$

0.43787

0.00035

0.90581

$-0.65477$

$-0.53333$

1.98727

2.30992

2.06368

2.67313

$\mathrm{H}=-705.824701$ a.u.

\section{$\mathbf{T S}_{\mathrm{A}}$}

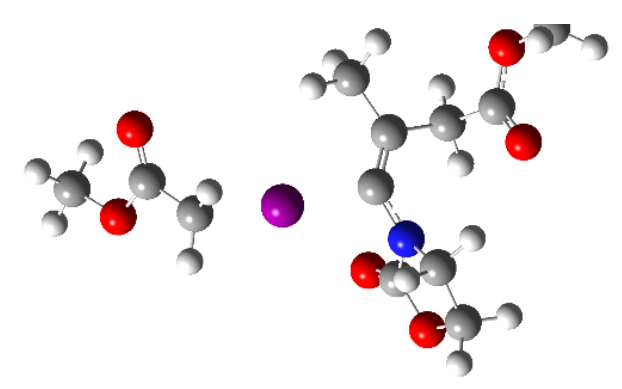




\begin{tabular}{|c|c|c|c|}
\hline $\mathrm{C}$ & 2.35916 & 3.30215 & -0.87984 \\
\hline 0 & 1.79359 & 3.59101 & 0.41297 \\
\hline $\mathrm{C}$ & 1.35196 & 2.45485 & 1.00575 \\
\hline $\mathrm{C}$ & 1.84748 & 1.90368 & -1.23687 \\
\hline $\mathrm{H}$ & 2.03030 & 4.07293 & -1.57281 \\
\hline $\mathrm{H}$ & 3.44605 & 3.32895 & -0.79096 \\
\hline $\mathrm{H}$ & 2.61608 & 1.27066 & -1.67615 \\
\hline $\mathrm{H}$ & 0.97288 & 1.93107 & -1.89223 \\
\hline $\mathrm{N}$ & 1.46850 & 1.40456 & 0.08338 \\
\hline 0 & 0.95119 & 2.38638 & 2.12879 \\
\hline $\mathrm{C}$ & 0.94273 & 0.16193 & 0.29374 \\
\hline $\mathrm{C}$ & 1.49471 & -0.91027 & 0.84088 \\
\hline $\mathrm{C}$ & 2.95290 & -0.84043 & 1.29690 \\
\hline $\mathrm{H}$ & 3.21469 & 0.17748 & 1.57600 \\
\hline $\mathrm{H}$ & 3.09237 & -1.49323 & 2.15772 \\
\hline $\mathrm{C}$ & 3.88899 & -1.25951 & 0.17883 \\
\hline 0 & 4.22255 & -0.54693 & -0.73595 \\
\hline 0 & 4.29246 & -2.53384 & 0.31272 \\
\hline $\mathrm{C}$ & 5.16138 & -3.03515 & -0.71968 \\
\hline $\mathrm{H}$ & 5.37433 & -4.06440 & -0.44579 \\
\hline $\mathrm{H}$ & 4.66558 & -2.98928 & -1.68837 \\
\hline $\mathrm{H}$ & 6.07914 & -2.45013 & -0.75906 \\
\hline $\mathrm{C}$ & 0.81551 & -2.22802 & 1.05489 \\
\hline $\mathrm{H}$ & -0.17201 & -2.25987 & 0.60410 \\
\hline $\mathrm{H}$ & 1.42160 & -3.03845 & 0.64178 \\
\hline $\mathrm{H}$ & 0.71356 & -2.42287 & 2.12659 \\
\hline $\mathrm{C}$ & -4.41432 & -0.87302 & -0.39098 \\
\hline 0 & -4.54852 & -2.07200 & -0.3093 \\
\hline 0 & -4.98568 & 0.00171 & 0.46574 \\
\hline $\mathrm{C}$ & -5.74834 & -0.57788 & 1.53294 \\
\hline $\mathrm{H}$ & -6.11558 & 0.26045 & 2.11867 \\
\hline $\mathrm{H}$ & -6.57892 & -1.16234 & 1.13771 \\
\hline $\mathrm{H}$ & -5.11886 & -1.22446 & 2.1439 \\
\hline $\mathrm{C}$ & -3.61075 & -0.18007 & -1.41727 \\
\hline I & -1.40886 & 0.11418 & -0.52667 \\
\hline $\mathrm{H}$ & -3.41632 & -0.78754 & -2.29004 \\
\hline $\mathrm{H}$ & -3.88794 & 0.84708 & -1.61665 \\
\hline
\end{tabular}

$\mathrm{H}=-1271.435893$ a.u.

Imaginary frequency $=i 148 \mathrm{~cm}^{-1}$

\section{A-I}

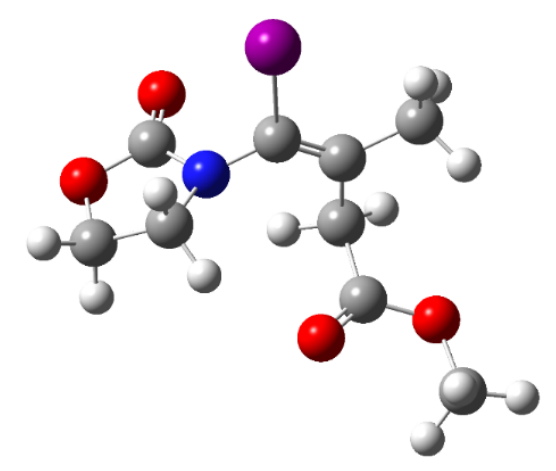




$\begin{array}{lr}\text { C } & -0.64363 \\ \mathrm{O} & -1.24727 \\ \mathrm{C} & -1.04335 \\ \mathrm{C} & -0.41480 \\ \mathrm{H} & -1.32189 \\ \mathrm{H} & 0.29503 \\ \mathrm{H} & 0.53921 \\ \mathrm{H} & -1.22485 \\ \mathrm{~N} & -0.42207 \\ \mathrm{O} & -1.34398 \\ \mathrm{C} & -0.30537 \\ \mathrm{C} & 0.77139 \\ \mathrm{C} & 1.96937 \\ \mathrm{H} & 1.66328 \\ \mathrm{H} & 2.43109 \\ \mathrm{C} & 2.99452 \\ \mathrm{O} & 2.88502 \\ \mathrm{O} & 4.04498 \\ \mathrm{C} & 5.06649 \\ \mathrm{H} & 5.81868 \\ \mathrm{H} & 4.65240 \\ \mathrm{H} & 5.49295 \\ \mathrm{C} & 0.92988 \\ \mathrm{H} & 0.15115 \\ \mathrm{H} & 1.90257 \\ \mathrm{H} & 0.90486 \\ \mathrm{I} & -2.05674 \\ \mathrm{H}=-1003.694315 \mathrm{a} u \\ \mathrm{H}\end{array}$

$\begin{array}{rr}3.24431 & -0.95280 \\ 3.22317 & 0.35423 \\ 2.01584 & 0.94455 \\ 1.77280 & -1.30681 \\ 3.75433 & -1.63297 \\ 3.79734 & -0.88890 \\ 1.60170 & -1.80204 \\ 1.35986 & -1.91696 \\ 1.17643 & 0.02513 \\ 1.76111 & 2.07472 \\ -0.19638 & 0.23624 \\ -0.78557 & 0.75998 \\ 0.06343 & 1.15901 \\ 1.08384 & 1.37314 \\ -0.36281 & 2.04898 \\ 0.12673 & 0.03918 \\ 0.80539 & -0.95131 \\ -0.67844 & 0.27483 \\ -0.69804 & -0.73924 \\ -1.39479 & -0.38068 \\ -1.03338 & -1.68918 \\ 0.29601 & -0.86609 \\ -2.25251 & 1.01408 \\ -2.84594 & 0.54358 \\ -2.59591 & 0.65352 \\ -2.44718 & 2.09080 \\ -1.32230 & -0.35196\end{array}$

$\mathbf{T S}_{\mathrm{B}}$

$\begin{array}{ll}\mathrm{C} & 4.40439 \\ \mathrm{O} & 4.24569 \\ \mathrm{C} & 3.26975 \\ \mathrm{C} & 3.12296 \\ \mathrm{H} & 4.54334 \\ \mathrm{H} & 5.29326 \\ \mathrm{H} & 3.30729 \\ \mathrm{H} & 2.38437 \\ \mathrm{~N} & 2.64883 \\ \mathrm{O} & 3.01228 \\ \mathrm{C} & 1.51003 \\ \mathrm{C} & 1.33202\end{array}$




$\begin{array}{rrrr}\mathrm{C} & -0.03252 & 1.81920 & 1.56031 \\ \mathrm{H} & -0.72934 & 1.02777 & 1.82449 \\ \mathrm{H} & 0.04904 & 2.48222 & 2.42774 \\ \mathrm{C} & -0.71077 & 2.63584 & 0.46837 \\ \mathrm{O} & -1.90490 & 2.70992 & 0.33272 \\ \mathrm{O} & 0.17100 & 3.29304 & -0.29895 \\ \mathrm{C} & -0.37750 & 4.08052 & -1.36932 \\ \mathrm{H} & 0.47903 & 4.50063 & -1.88835 \\ \mathrm{H} & -0.95912 & 3.45050 & -2.04046 \\ \mathrm{H} & -1.01553 & 4.86970 & -0.97293 \\ \mathrm{C} & 2.50846 & 2.14773 & 1.57416 \\ \mathrm{H} & 3.46101 & 1.65694 & 1.38990 \\ \mathrm{H} & 2.46874 & 3.05957 & 0.97469 \\ \mathrm{H} & 2.45967 & 2.43961 & 2.62688 \\ \mathrm{C} & -3.60419 & -1.34632 & -0.11877 \\ \mathrm{O} & -4.20317 & -1.35999 & 0.93120 \\ \mathrm{O} & -3.79977 & -0.42910 & -1.08582 \\ \mathrm{C} & -4.71248 & 0.63585 & -0.77005 \\ \mathrm{H} & -4.82449 & 1.20392 & -1.68952 \\ \mathrm{H} & -5.67134 & 0.23428 & -0.44545 \\ \mathrm{H} & -4.29124 & 1.26611 & 0.01219 \\ \mathrm{C} & -2.55424 & -2.31734 & -0.49980 \\ \mathrm{I} & -0.48328 & -1.36787 & 0.16811 \\ \mathrm{H} & -2.58005 & -3.22621 & 0.08650 \\ \mathrm{H} & -2.40703 & -2.45476 & -1.56324\end{array}$

\section{$\mathrm{H}=-1271.433461$ a.u.}

\section{Imaginary frequency $=i 142 \mathrm{~cm}^{-1}$}

\section{B-I}

$\begin{array}{rrrr}\mathrm{C} & 3.92273 & 1.15896 & 0.00503 \\ \mathrm{O} & 3.18659 & 1.65445 & -1.12521 \\ \mathrm{C} & 1.85384 & 1.44400 & -0.94927 \\ \mathrm{C} & 2.95207 & 0.21924 & 0.72615 \\ \mathrm{H} & 4.81649 & 0.65902 & -0.36127 \\ \mathrm{H} & 4.21068 & 2.00769 & 0.62836 \\ \mathrm{H} & 3.01180 & 0.30555 & 1.81091 \\ \mathrm{H} & 3.10087 & -0.82904 & 0.44939 \\ \mathrm{~N} & 1.67600 & 0.70985 & 0.22304 \\ \mathrm{O} & 1.00945 & 1.84765 & -1.69273 \\ \mathrm{C} & 0.44659 & 0.13892 & 0.55153 \\ \mathrm{C} & -0.56470 & 0.81785 & 1.10268 \\ \mathrm{C} & -1.93257 & 0.23241 & 1.35929 \\ \mathrm{H} & -1.91706 & -0.85204 & 1.29919 \\ \mathrm{H} & -2.26599 & 0.53539 & 2.35348\end{array}$




$\begin{array}{lr}\text { C } & -2.95274 \\ \text { O } & -3.50277 \\ \text { O } & -3.14923 \\ \mathrm{C} & -4.03989 \\ \mathrm{H} & -4.07308 \\ \mathrm{H} & -5.03107 \\ \mathrm{H} & -3.65509 \\ \mathrm{C} & -0.41166 \\ \mathrm{H} & 0.60137 \\ \mathrm{H} & -1.09218 \\ \mathrm{H} & -0.69214 \\ \mathrm{I} & 0.28791\end{array}$

$H=-1003.691987$ a.u.

$\mathbf{C}^{\bullet}$

$\begin{array}{rr}0.78272 & 0.37063 \\ 1.84898 & 0.47836 \\ -0.06449 & -0.64965 \\ 0.38888 & -1.68466 \\ -0.41737 & -2.41166 \\ 0.58107 & -1.27586 \\ 1.30023 & -2.14008 \\ 2.26135 & 1.50444 \\ 2.62582 & 1.36343 \\ 2.88639 & 0.92271 \\ 2.38312 & 2.55428 \\ -1.94554 & -0.01425\end{array}$

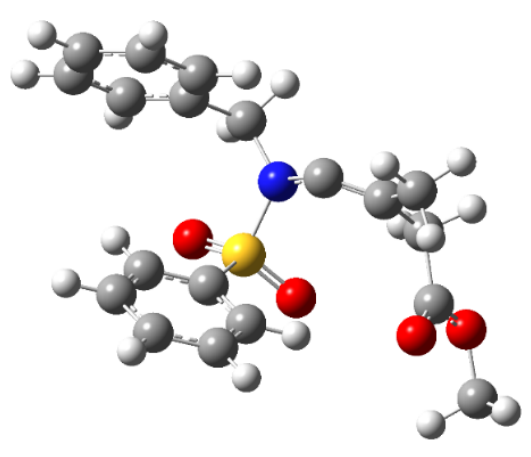

$-1.18131$

$-1.27263$

$-1.42073$

$-1.34036$

0.16556

0.02336

0.21494

1.59466

2.20359

2.08520

3.32241

1.81358

3.20686

1.60016

3.82251

3.80475

3.60057

4.69740

$-2.04765$

$-3.00562$

$-2.19281$

$-1.54357$

$-1.47202$

$-1.16910$

$-1.02649$

$-1.76148$

$-0.72918$

$-1.21454$
$-0.37711$

0.85242

1.32451

2.80284

$-1.38230$

$-2.49207$

$-1.59068$

$-0.43167$

0.38148

$-0.52324$

1.11889

0.43161

0.21606

$-1.16899$

1.03721

1.75417

0.14785

1.61165

$-0.67956$

$-0.21634$

$-1.75592$

$-0.18741$

1.17745

$-1.10051$

1.61793

1.89659

$-0.66155$

$-2.16129$ 


$\begin{array}{rrrr}\mathrm{C} & 5.18550 & -0.65482 & 0.69899 \\ \mathrm{H} & 4.41566 & -0.97509 & 2.67961 \\ \mathrm{H} & 5.66922 & -0.44506 & -1.38352 \\ \mathrm{H} & 6.15370 & -0.31314 & 1.04302 \\ \mathrm{H} & -2.62178 & -0.54262 & 3.01375 \\ \mathrm{H} & -2.34492 & -2.27703 & 3.16289 \\ \mathrm{H} & -0.99290 & -1.14843 & 3.36292 \\ \mathrm{C} & -2.81944 & -1.72218 & 0.43391 \\ \mathrm{H} & -3.38669 & -2.56557 & 0.84298 \\ \mathrm{H} & -2.50466 & -2.00684 & -0.56886 \\ \mathrm{C} & -3.78873 & -0.56192 & 0.28862 \\ \mathrm{O} & -3.72455 & 0.49681 & 0.86157 \\ \mathrm{O} & -4.76766 & -0.87971 & -0.57498 \\ \mathrm{C} & -5.73636 & 0.14807 & -0.83818 \\ \mathrm{H} & -5.25039 & 1.02327 & -1.26738 \\ \mathrm{H} & -6.43589 & -0.28575 & -1.54740 \\ \mathrm{H} & -6.24923 & 0.43537 & 0.07919\end{array}$

$H=-1489.873504$ a.u.

\section{$\mathbf{C}^{\bullet} \rightarrow \mathbf{D}^{\bullet}: \mathbf{T S}_{\text {inv }}$}

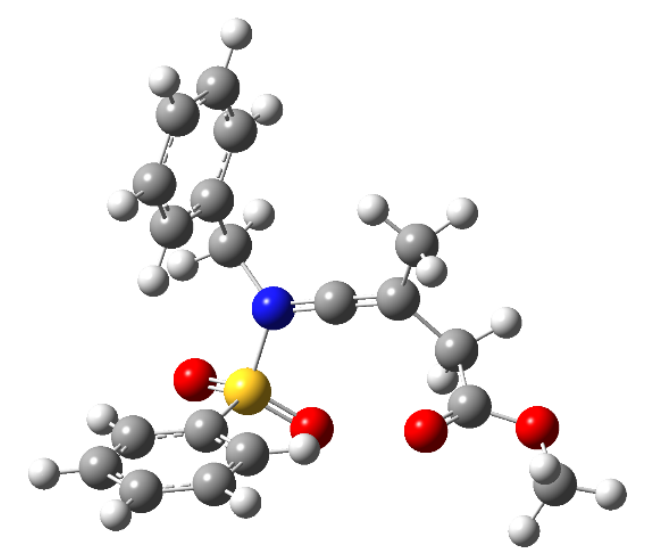

$\begin{array}{rrrr}\mathrm{N} & -0.63079 & -0.21953 & 1.24638 \\ \mathrm{C} & 0.28674 & -1.05892 & 0.80938 \\ \mathrm{C} & 1.22132 & -1.86992 & 0.38622 \\ \mathrm{C} & 0.95615 & -3.01066 & -0.57745 \\ \mathrm{~S} & -0.11952 & 1.39579 & 1.67929 \\ \mathrm{O} & -1.09749 & 1.91363 & 2.60015 \\ \mathrm{O} & 1.26809 & 1.27972 & 2.02957 \\ \mathrm{C} & -0.24221 & 2.30366 & 0.15668 \\ \mathrm{C} & 0.70298 & 2.08992 & -0.84539 \\ \mathrm{C} & -1.28290 & 3.21361 & -0.00618 \\ \mathrm{C} & 0.58731 & 2.80093 & -2.03265 \\ \mathrm{H} & 1.51003 & 1.38199 & -0.71044 \\ \mathrm{C} & -1.37860 & 3.92294 & -1.19736 \\ \mathrm{H} & -1.99134 & 3.36962 & 0.79514 \\ \mathrm{C} & -0.44748 & 3.71479 & -2.20908 \\ \mathrm{H} & 1.31358 & 2.64000 & -2.81860 \\ \mathrm{H} & -2.17783 & 4.64059 & -1.33075 \\ \mathrm{H} & -0.52548 & 4.26921 & -3.13585 \\ \mathrm{C} & -2.01263 & -0.65673 & 1.57919 \\ \mathrm{H} & -1.95398 & -1.37110 & 2.40309\end{array}$




$\begin{array}{lrrr}\mathrm{H} & -2.53890 & 0.22176 & 1.94839 \\ \mathrm{C} & -2.73602 & -1.27588 & 0.40839 \\ \mathrm{C} & -3.16230 & -2.60046 & 0.46997 \\ \mathrm{C} & -3.00935 & -0.53467 & -0.74265 \\ \mathrm{C} & -3.85324 & -3.17634 & -0.59197 \\ \mathrm{H} & -2.95164 & -3.18794 & 1.35599 \\ \mathrm{C} & -3.69357 & -1.10744 & -1.80571 \\ \mathrm{H} & -2.67826 & 0.49461 & -0.81040 \\ \mathrm{C} & -4.11965 & -2.43080 & -1.73266 \\ \mathrm{H} & -4.17800 & -4.20725 & -0.52735 \\ \mathrm{H} & -3.89762 & -0.52051 & -2.69246 \\ \mathrm{H} & -4.65459 & -2.87624 & -2.56189 \\ \mathrm{H} & 1.51899 & -2.86996 & -1.50540 \\ \mathrm{H} & 1.27563 & -3.96483 & -0.14418 \\ \mathrm{H} & -0.10020 & -3.08315 & -0.82884 \\ \mathrm{C} & 2.67272 & -1.69389 & 0.80915 \\ \mathrm{H} & 3.10884 & -2.64485 & 1.12322 \\ \mathrm{H} & 2.73583 & -1.01041 & 1.65913 \\ \mathrm{C} & 3.55365 & -1.09516 & -0.27282 \\ \mathrm{O} & 3.19942 & -0.31316 & -1.12003 \\ \mathrm{O} & 4.82700 & -1.52004 & -0.15190 \\ \mathrm{C} & 5.77280 & -0.95176 & -1.07170 \\ \mathrm{H} & 5.81606 & 0.13064 & -0.95453 \\ \mathrm{H} & 6.73001 & -1.40094 & -0.82128 \\ \mathrm{H} & 5.49629 & -1.18776 & -2.09876\end{array}$

$H=-1489.861173$ a.u.

Imaginary frequency $=i 161 \mathrm{~cm}^{-1}$

$\mathbf{D}^{\bullet}$

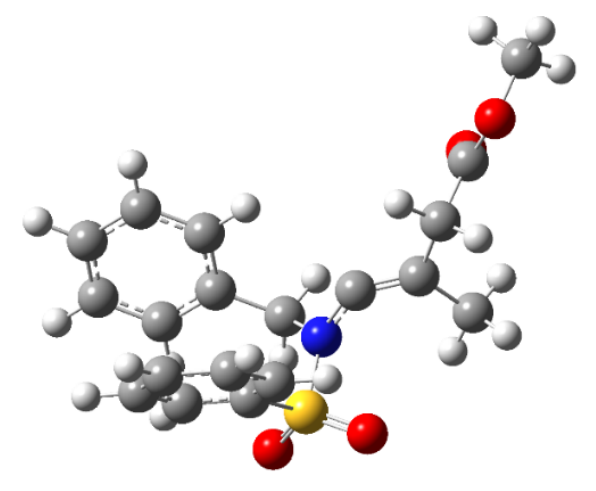

$\begin{array}{rrrr}\mathrm{N} & -0.04507 & -0.14889 & -1.18509 \\ \mathrm{C} & 1.05196 & -0.25882 & -0.37136 \\ \mathrm{C} & 2.29078 & -0.67031 & -0.57215 \\ \mathrm{C} & 2.79835 & -1.16584 & -1.90366 \\ \mathrm{~S} & -1.11662 & -1.46049 & -1.30500 \\ \mathrm{O} & -2.10270 & -1.05730 & -2.27051 \\ \mathrm{O} & -0.30669 & -2.62852 & -1.49926 \\ \mathrm{C} & -1.90201 & -1.61421 & 0.28306 \\ \mathrm{C} & -1.29195 & -2.38429 & 1.26997\end{array}$




\begin{tabular}{|c|c|c|c|}
\hline $\mathrm{C}$ & -3.11508 & -0.97386 & 0.51526 \\
\hline $\mathrm{C}$ & -1.90558 & -2.50255 & 2.51015 \\
\hline $\mathrm{H}$ & -0.36294 & -2.89331 & 1.05748 \\
\hline $\mathrm{C}$ & -3.72087 & -1.10370 & 1.75819 \\
\hline $\mathrm{H}$ & -3.57725 & -0.39266 & -0.26927 \\
\hline $\mathrm{C}$ & -3.11641 & -1.86280 & 2.75457 \\
\hline $\mathrm{H}$ & -1.44127 & -3.10187 & 3.28267 \\
\hline $\mathrm{H}$ & -4.66698 & -0.61301 & 1.94593 \\
\hline $\mathrm{H}$ & -3.59318 & -1.96177 & 3.72159 \\
\hline $\mathrm{C}$ & -0.45833 & 1.18782 & -1.68018 \\
\hline $\mathrm{H}$ & 0.47773 & 1.71610 & -1.86520 \\
\hline $\mathrm{H}$ & -0.95510 & 1.03768 & -2.63524 \\
\hline $\mathrm{C}$ & -1.34495 & 1.99609 & -0.75829 \\
\hline $\mathrm{C}$ & -0.90409 & 2.39593 & 0.50452 \\
\hline $\mathrm{C}$ & -2.61407 & 2.38682 & -1.18182 \\
\hline $\mathrm{C}$ & -1.71926 & 3.16231 & 1.32669 \\
\hline $\mathrm{H}$ & 0.08091 & 2.10033 & 0.84466 \\
\hline $\mathrm{C}$ & -3.42996 & 3.16088 & -0.36257 \\
\hline $\mathrm{H}$ & -2.96784 & 2.07813 & -2.15830 \\
\hline $\mathrm{C}$ & -2.98476 & 3.54860 & 0.89482 \\
\hline $\mathrm{H}$ & -1.36459 & 3.46488 & 2.30407 \\
\hline $\mathrm{H}$ & -4.41211 & 3.45884 & -0.70801 \\
\hline $\mathrm{H}$ & -3.61725 & 4.15105 & 1.53471 \\
\hline $\mathrm{H}$ & 3.64918 & -0.56358 & -2.22966 \\
\hline $\mathrm{H}$ & 3.13559 & -2.20269 & -1.81405 \\
\hline $\mathrm{H}$ & 2.02296 & -1.13040 & -2.66512 \\
\hline $\mathrm{C}$ & 3.26816 & -0.67648 & 0.57482 \\
\hline $\mathrm{H}$ & 3.72660 & -1.66368 & 0.68902 \\
\hline $\mathrm{H}$ & 2.75516 & -0.46411 & 1.51527 \\
\hline C & 4.38895 & 0.33905 & 0.43917 \\
\hline O & 4.45479 & 1.22052 & -0.37754 \\
\hline O & 5.32076 & 0.13841 & 1.39311 \\
\hline C & 6.42140 & 1.06189 & 1.40169 \\
\hline $\mathrm{H}$ & 6.06503 & 2.07991 & 1.55558 \\
\hline $\mathrm{H}$ & 7.05693 & 0.75106 & 2.22635 \\
\hline $\mathrm{H}$ & 6.96666 & 1.01508 & 0.45966 \\
\hline
\end{tabular}

$H=-1489.872021$ a.u.

\section{$\mathbf{T S}_{\mathrm{C}}$}

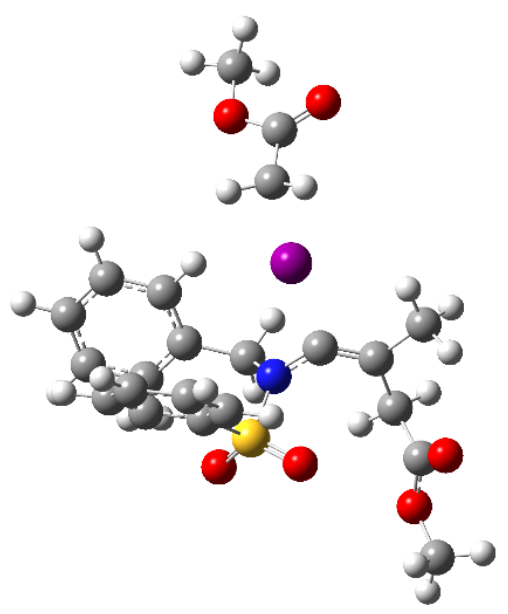




\begin{tabular}{|c|c|c|c|}
\hline $\mathrm{N}$ & -1.11350 & 0.32646 & -0.76705 \\
\hline C & -0.81487 & -1.00026 & -0.59937 \\
\hline $\mathrm{C}$ & -1.53685 & -2.08680 & -0.86481 \\
\hline $\mathrm{C}$ & -1.10760 & -3.50162 & -0.62812 \\
\hline $\mathrm{S}$ & -2.12608 & 1.12541 & 0.35599 \\
\hline 0 & -2.64448 & 2.25711 & -0.36136 \\
\hline 0 & -2.99529 & 0.12511 & 0.90608 \\
\hline $\mathrm{C}$ & -1.08064 & 1.70165 & 1.66880 \\
\hline C & -0.89320 & 0.88947 & 2.78419 \\
\hline $\mathrm{C}$ & -0.49729 & 2.96243 & 1.58314 \\
\hline $\mathrm{C}$ & -0.10027 & 1.35022 & 3.82644 \\
\hline $\mathrm{H}$ & -1.37319 & -0.07709 & 2.83501 \\
\hline $\mathrm{C}$ & 0.29265 & 3.41025 & 2.63326 \\
\hline $\mathrm{H}$ & -0.66763 & 3.58214 & 0.71478 \\
\hline $\mathrm{C}$ & 0.49242 & 2.60610 & 3.75076 \\
\hline $\mathrm{H}$ & 0.04963 & 0.72852 & 4.69943 \\
\hline $\mathrm{H}$ & 0.74801 & 4.39038 & 2.57891 \\
\hline $\mathrm{H}$ & 1.10709 & 2.96214 & 4.56789 \\
\hline $\mathrm{C}$ & -0.70649 & 1.02943 & -2.02414 \\
\hline $\mathrm{H}$ & -0.16369 & 0.27129 & -2.58639 \\
\hline $\mathrm{H}$ & -1.61625 & 1.26786 & -2.57520 \\
\hline C & 0.13952 & 2.27728 & -1.89672 \\
\hline $\mathrm{C}$ & 1.48947 & 2.20616 & -1.54936 \\
\hline $\mathrm{C}$ & -0.41075 & 3.52246 & -2.20140 \\
\hline $\mathrm{C}$ & 2.26583 & 3.35667 & -1.49141 \\
\hline $\mathrm{H}$ & 1.93606 & 1.24775 & -1.31887 \\
\hline $\mathrm{C}$ & 0.36604 & 4.67452 & -2.14960 \\
\hline $\mathrm{H}$ & -1.45775 & 3.59035 & -2.46713 \\
\hline $\mathrm{C}$ & 1.70657 & 4.59455 & -1.79087 \\
\hline $\mathrm{H}$ & 3.31110 & 3.28389 & -1.21847 \\
\hline $\mathrm{H}$ & -0.07715 & 5.63279 & -2.38998 \\
\hline $\mathrm{H}$ & 2.31378 & 5.49028 & -1.75106 \\
\hline $\mathrm{H}$ & -1.82816 & -3.99098 & 0.02912 \\
\hline $\mathrm{H}$ & -1.11019 & -4.05027 & -1.57525 \\
\hline $\mathrm{H}$ & -0.11666 & -3.56928 & -0.19151 \\
\hline $\mathrm{C}$ & -2.90617 & -1.92739 & -1.50288 \\
\hline $\mathrm{H}$ & -2.97623 & -2.61223 & -2.35597 \\
\hline $\mathrm{H}$ & -3.06402 & -0.92307 & -1.88553 \\
\hline C & -4.07074 & -2.27223 & -0.58376 \\
\hline 0 & -4.04735 & -3.05071 & 0.33289 \\
\hline 0 & -5.17241 & -1.61077 & -0.97540 \\
\hline C & -6.35554 & -1.84435 & -0.19332 \\
\hline $\mathrm{H}$ & -6.18795 & -1.54106 & 0.83903 \\
\hline $\mathrm{H}$ & -7.13065 & -1.23524 & -0.64994 \\
\hline $\mathrm{H}$ & -6.62968 & -2.89850 & -0.21918 \\
\hline C & 4.53556 & -2.20416 & 0.23659 \\
\hline O & 4.74519 & -3.33487 & -0.13802 \\
\hline 0 & 5.12602 & -1.11750 & -0.31822 \\
\hline C & 6.01085 & -1.38574 & -1.41357 \\
\hline $\mathrm{H}$ & 6.38283 & -0.41629 & -1.73473 \\
\hline $\mathrm{H}$ & 6.83476 & -2.02410 & -1.09506 \\
\hline $\mathrm{H}$ & 5.47669 & -1.87854 & -2.22583 \\
\hline $\mathrm{C}$ & 3.62054 & -1.82845 & 1.32620 \\
\hline $\mathrm{H}$ & 3.82753 & -0.88799 & 1.81960 \\
\hline $\mathrm{H}$ & 3.35793 & -2.65213 & 1.97451 \\
\hline I & 1.47430 & -1.30774 & 0.31636 \\
\hline
\end{tabular}

$\mathrm{H}=-2055.474256$ a.u. 
Imaginary frequency $=i 153 \mathrm{~cm}^{-1}$

\section{C-I}

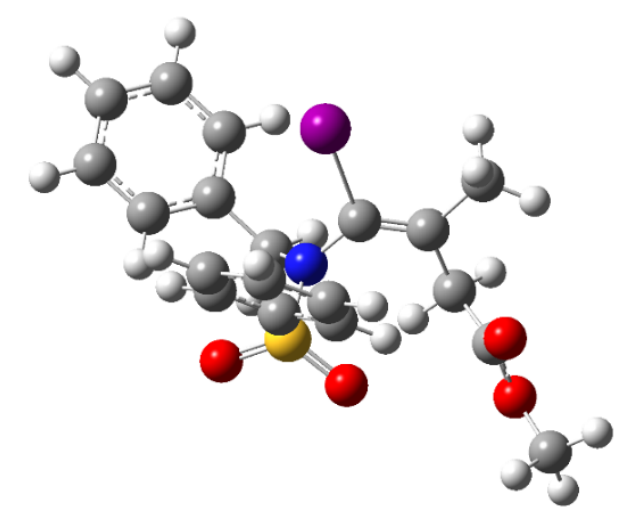

$$
0.10687
$$

$-0.26188$

$-1.35031$

$-1.69081$

$-0.63261$

0.19192

$-2.03015$

$-0.52765$

$-1.60637$

0.61583

$-1.52695$

$-2.48708$

0.67829

1.43534

$-0.38999$

$-2.35769$

1.56014

$-0.33630$

1.01851

0.72214

0.80599

2. 50001

3.20380

3. 19760

4.57259

2.67642

4.56415

2.66422

5. 25548

5.10304

5.09364

6.32148

$-1.71296$

$-2.66674$

$-0.94640$

$-2.30980$

$-2.49253$

$-1.92483$

$-3.65863$

$$
\begin{array}{r}
-0.66299 \\
-0.75162 \\
-1.36545 \\
-1.42572 \\
0.46270 \\
0.51371 \\
0.14349 \\
2.00651 \\
2.42868 \\
2.78726 \\
3.65502 \\
1.80764 \\
4.01285 \\
2.44190 \\
4.44434 \\
3.99319 \\
4.63116 \\
5.40095 \\
-1.67863 \\
-2.64038 \\
-1.70654 \\
-1.46473 \\
-2.27775 \\
-0.49040 \\
-2.11550 \\
-3.04351 \\
-0.32419 \\
0.12888 \\
-1.13447 \\
-2.75413 \\
0.43340 \\
-1.00538 \\
-0.43100 \\
-1.88311 \\
-2.00852 \\
-2.07671 \\
-3.08827 \\
-2.17748 \\
-1.38194
\end{array}
$$

$-0.74473$

0.60182

1.08523

2.54901

$-1.76155$

$-2.94136$

$-1.89564$

$-0.89352$

$-0.12193$

$-1.03366$

0.52616

$-0.03418$

$-0.38634$

$-1.64752$

0.39405

1. 13169

$-0.49223$

0.89834

$-1.33901$

$-0.91768$

$-2.40564$

$-1.12088$

$-0.23472$

$-1.83805$

$-0.05202$

0.32222

$-1.65509$

$-2.54774$

$-0.75954$

0.64313

$-2.21953$

$-0.62014$

2.99366

2. 70075

3. 09781

0.16464

0.54290

$-0.84677$

0.06511 


$\begin{array}{rrrr}\mathrm{O} & -3.98567 & -0.38780 & 0.66107 \\ \mathrm{O} & -4.45948 & -2.04636 & -0.78198 \\ \mathrm{C} & -5.75241 & -1.46369 & -1.01626 \\ \mathrm{H} & -5.64357 & -0.46844 & -1.44488 \\ \mathrm{H} & -6.24850 & -2.12900 & -1.71735 \\ \mathrm{H} & -6.31605 & -1.39645 & -0.08638 \\ \mathrm{I} & 1.11702 & 0.17067 & 2.00190\end{array}$

$H=-1787.730770$ a.u.

\section{$\mathbf{T S}_{\mathrm{D}}$}

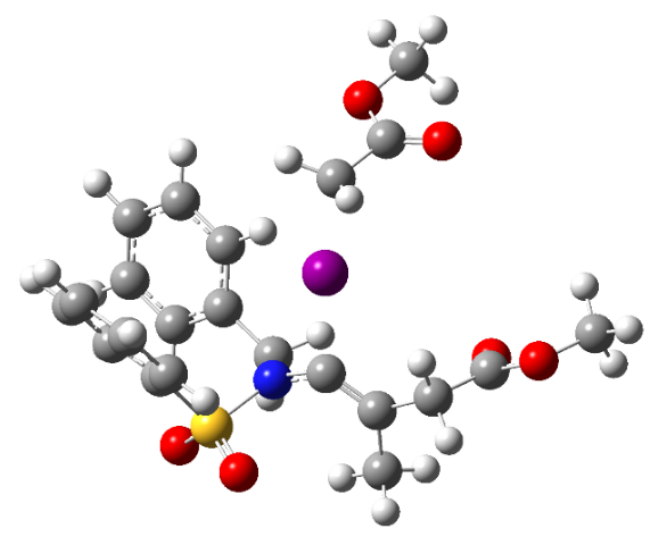

\begin{tabular}{lr}
$\mathrm{N}$ & 1.04771 \\
$\mathrm{C}$ & -0.23802 \\
$\mathrm{C}$ & -1.08237 \\
$\mathrm{C}$ & -0.67065 \\
$\mathrm{~S}$ & 2.33710 \\
$\mathrm{O}$ & 3.38967 \\
$\mathrm{O}$ & 1.78717 \\
$\mathrm{C}$ & 2.84797 \\
$\mathrm{C}$ & 2.30927 \\
$\mathrm{C}$ & 3.80424 \\
$\mathrm{C}$ & 2.73252 \\
$\mathrm{H}$ & 1.58324 \\
$\mathrm{C}$ & 4.21784 \\
$\mathrm{H}$ & 4.22149 \\
$\mathrm{C}$ & 3.68255 \\
$\mathrm{H}$ & 2.32235 \\
$\mathrm{H}$ & 4.96277 \\
$\mathrm{H}$ & 4.01158 \\
$\mathrm{C}$ & 1.30377 \\
$\mathrm{H}$ & 0.30956 \\
$\mathrm{H}$ & 1.75184 \\
$\mathrm{C}$ & 2.15798 \\
$\mathrm{C}$ & 1.67602 \\
$\mathrm{C}$ & 3.42821 \\
$\mathrm{C}$ & 2.45429 \\
$\mathrm{H}$ & 0.68864 \\
$\mathrm{C}$ & 4.20662 \\
$\mathrm{H}$ & 3.81334 \\
$\mathrm{C}$ & 2.06657 \\
$\mathrm{H}$ & \\
$\mathrm{H}$ & \\
\hline
\end{tabular}

$$
\begin{array}{r}
-1.45596 \\
-1.51093 \\
-2.53556 \\
-3.90790 \\
-1.90536 \\
-2.30932 \\
-2.80483 \\
-0.43682 \\
-0.16901 \\
0.39836 \\
0.95981 \\
-0.84344 \\
1.52410 \\
0.16493 \\
1.80545 \\
1.17495 \\
2.17830 \\
2.68305 \\
-1.25038 \\
-1.15499 \\
-2.16474 \\
-0.06620 \\
1.23952 \\
-0.27312 \\
2.31441 \\
1.41741 \\
0.80142 \\
-1.28168 \\
2.09866 \\
3.32150 \\
0.62331
\end{array}
$$$$
0.41939
$$$$
-0.04459
$$$$
-0.08849
$$$$
0.38770
$$$$
-0.61105
$$$$
0.28002
$$$$
-1.58368
$$$$
-1.46900
$$$$
-2.72442
$$$$
-0.89858
$$$$
-3.41295
$$$$
-3.15426
$$$$
-1.59774
$$$$
0.07006
$$$$
-2.85058
$$$$
-4.39102
$$$$
-1.16407
$$$$
-3.39266
$$$$
1.87849
$$$$
2.31215
$$$$
\text { 2. } 26612
$$$$
2.26970
$$$$
2.16498
$$$$
2.80711
$$$$
2.57478
$$$$
\text { 1. } 75858
$$$$
\text { 3. } 22401
$$$$
2.88778
$$$$
3.10559
$$$$
2.48608
$$

3.64107 


$\begin{array}{rrrr}\mathrm{H} & 4.32566 & 2.93688 & 3.43077 \\ \mathrm{H} & -1.36849 & -4.25064 & 1.15508 \\ \mathrm{H} & -0.71227 & -4.61385 & -0.44597 \\ \mathrm{H} & 0.33706 & -3.92715 & 0.79283 \\ \mathrm{C} & -2.49119 & -2.44374 & -0.60102 \\ \mathrm{H} & -2.71727 & -3.31175 & -1.22731 \\ \mathrm{H} & -2.64100 & -1.56388 & -1.22450 \\ \mathrm{C} & -3.53111 & -2.39778 & 0.50622 \\ \mathrm{O} & -3.30585 & -2.39056 & 1.68901 \\ \mathrm{O} & -4.76625 & -2.35922 & -0.02148 \\ \mathrm{C} & -5.85256 & -2.26704 & 0.91570 \\ \mathrm{H} & -5.76674 & -1.35582 & 1.50602 \\ \mathrm{H} & -6.75567 & -2.24604 & 0.31240 \\ \mathrm{H} & -5.85553 & -3.12852 & 1.58256 \\ \mathrm{C} & -3.13729 & 3.13642 & -0.51685 \\ \mathrm{O} & -4.30320 & 2.81695 & -0.56529 \\ \mathrm{O} & -2.61094 & 3.87268 & 0.49108 \\ \mathrm{C} & -3.52224 & 4.23424 & 1.53682 \\ \mathrm{H} & -2.93745 & 4.80976 & 2.24960 \\ \mathrm{H} & -4.34184 & 4.83455 & 1.14232 \\ \mathrm{H} & -3.93318 & 3.34325 & 2.01135 \\ \mathrm{C} & -2.11825 & 2.77742 & -1.51576 \\ \mathrm{H} & -1.25238 & 3.42421 & -1.56842 \\ \mathrm{H} & -2.53131 & 2.46497 & -2.46422 \\ \mathrm{I} & -1.06456 & 0.72357 & -0.75387\end{array}$

$\mathrm{H}=-2055.476275$ a.u.

Imaginary frequency $=i 154 \mathrm{~cm}^{-1}$

\section{D-I}

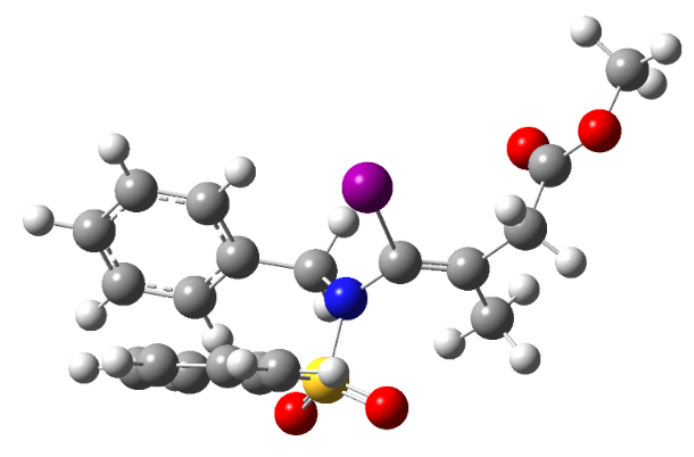

$\begin{array}{rrrr}\mathrm{N} & 0.17315 & 0.42079 & 0.90914 \\ \mathrm{C} & -1.06107 & -0.07815 & 0.48675 \\ \mathrm{C} & -2.18276 & -0.05624 & 1.21327 \\ \mathrm{C} & -2.21062 & 0.51028 & 2.60716 \\ \mathrm{~S} & 1.18175 & -0.53759 & 1.85906 \\ \mathrm{O} & 1.96788 & 0.39046 & 2.62664 \\ \mathrm{O} & 0.34985 & -1.52185 & 2.49324 \\ \mathrm{C} & 2.27628 & -1.41435 & 0.76213 \\ \mathrm{C} & 1.92317 & -2.69051 & 0.33231 \\ \mathrm{C} & 3.48341 & -0.83553 & 0.38181 \\ \mathrm{C} & 2.78760 & -3.38795 & -0.50099 \\ \mathrm{H} & 0.99334 & -3.13097 & 0.66141\end{array}$




\begin{tabular}{|c|c|c|c|}
\hline C & 4.33963 & -1.54329 & -0.45129 \\
\hline $\mathrm{H}$ & 3.74990 & 0.14758 & 0.74131 \\
\hline C & 3.99205 & -2.81502 & -0.89467 \\
\hline $\mathrm{H}$ & 2.52118 & -4.38153 & -0.83745 \\
\hline $\mathrm{H}$ & 5.28124 & -1.10090 & -0.74937 \\
\hline $\mathrm{H}$ & 4.66463 & -3.36354 & -1.54204 \\
\hline $\mathrm{C}$ & 0.46750 & 1.87396 & 0.74041 \\
\hline $\mathrm{H}$ & -0.41476 & 2.27234 & 0.24081 \\
\hline $\mathrm{H}$ & 0.51903 & 2.32918 & 1.72911 \\
\hline $\mathrm{C}$ & 1.70946 & 2.24119 & -0.04078 \\
\hline $\mathrm{C}$ & 1.79792 & 2.01816 & -1.41580 \\
\hline C & 2.77264 & 2.87058 & 0.60569 \\
\hline C & 2.92783 & 2.40522 & -2.12427 \\
\hline $\mathrm{H}$ & 0.97910 & 1.53689 & -1.93540 \\
\hline $\mathrm{C}$ & 3.90334 & 3.26461 & -0.10222 \\
\hline $\mathrm{H}$ & 2.71853 & 3.04056 & 1.67364 \\
\hline $\mathrm{C}$ & 3.98463 & 3.03019 & -1.46948 \\
\hline $\mathrm{H}$ & 2.98086 & 2.22449 & -3.19063 \\
\hline $\mathrm{H}$ & 4.71917 & 3.75297 & 0.41599 \\
\hline $\mathrm{H}$ & 4.86333 & 3.33616 & -2.02346 \\
\hline $\mathrm{H}$ & -1.26668 & 0.95398 & 2.90677 \\
\hline $\mathrm{H}$ & -2.99491 & 1.26807 & 2.67415 \\
\hline $\mathrm{H}$ & -2.44933 & -0.28315 & 3.32059 \\
\hline C & -3.50551 & -0.58354 & 0.73511 \\
\hline $\mathrm{H}$ & -4.01942 & -1.08919 & 1.55826 \\
\hline $\mathrm{H}$ & -3.39564 & -1.32211 & -0.05741 \\
\hline $\mathrm{C}$ & -4.43883 & 0.50461 & 0.22906 \\
\hline O & -4.21212 & 1.68651 & 0.22724 \\
\hline O & -5.58347 & -0.03887 & -0.22165 \\
\hline $\mathrm{C}$ & -6.56124 & 0.88314 & -0.73275 \\
\hline $\mathrm{H}$ & -6.15493 & 1.43875 & -1.57692 \\
\hline $\mathrm{H}$ & -7.40194 & 0.27099 & -1.04687 \\
\hline $\mathrm{H}$ & -6.86622 & 1.58449 & 0.04307 \\
\hline I & -1.08974 & -0.85176 & -1.55258 \\
\hline
\end{tabular}

\section{Energy profile for the regioselectivity of the addition of $\alpha$-methoxycarbonyl radical to}

ynamide 1d (enthalpies are given in $\mathrm{kcal} / \mathrm{mol}$, calculations were performed at the B3LYP/6-

$31+\mathrm{G}(\mathrm{d}, \mathrm{p})$ level of theory). 


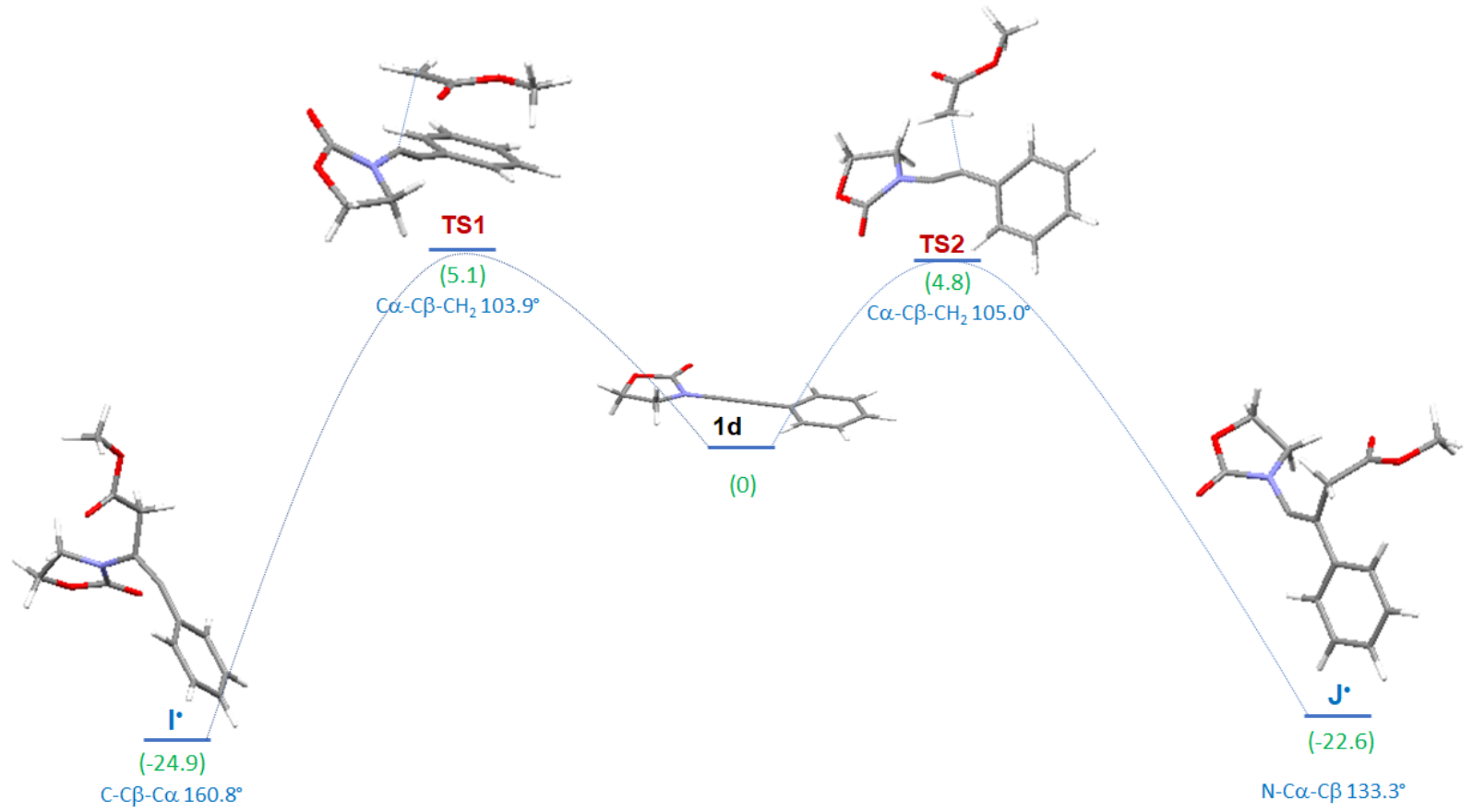

1d

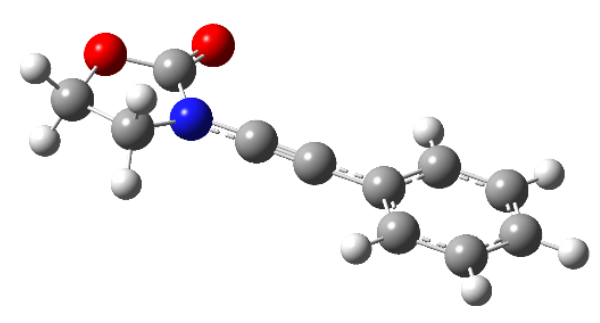

$-4.19900$

$-0.95662$

$-0.13383$

$-4.12754$

0.46695

0.08569

$-2.83339$

0.89579

0.01378

$-2.77524$

$-1.46985$

0.13386

$-1.36752$

0.54754

$-4.50960$

$-1.12951$

$-1.16929$

$-2.46486$

$-2.24560$

$-0.56991$

$-2.64270$

$-1.83877$

1.15834

$-2.01027$

$-0.23613$

$-0.07049$

2.04967

0.02845

$-0.66602$

$-0.17825$

$-0.04320$

0.54771

$-0.15476$

$-0.03358$

1.97344

$-0.09104$

$-0.01677$

2.74954

$-1.26772$

$-0.00025$

2.62782

1.15816

$-0.01723$

$-1.19281$

0.01624

2.25326

$-2.23313$

$-0.00030$

4.02016

1.22121

$-0.00229$

2.03491

2.06696

$-0.02930$

0.04943

0.01492

4.72666

$-2.10790$

0.02955 

$\mathrm{H}$
4.51091
2.19022
$-0.00334$
$\mathrm{H}$
5.86787
0.10390
0.02712

$\mathrm{H}=-629.579041$ a.u.

\section{TS2}

$\begin{array}{lr}\text { C } & -4.22001 \\ \mathrm{O} & -4.18806 \\ \mathrm{C} & -2.91652 \\ \mathrm{C} & -2.78662 \\ \mathrm{H} & -4.95823 \\ \mathrm{H} & -4.52162 \\ \mathrm{H} & -2.44498 \\ \mathrm{H} & -2.64475 \\ \mathrm{~N} & -2.05235 \\ \mathrm{O} & -2.60231 \\ \mathrm{C} & -0.72684 \\ \mathrm{C} & 0.45789 \\ \mathrm{C} & 1.84645 \\ \mathrm{C} & 2.86712 \\ \mathrm{C} & 2.18095 \\ \mathrm{C} & 4.18828 \\ \mathrm{H} & 2.62142 \\ \mathrm{C} & 3.50537 \\ \mathrm{H} & 1.39574 \\ \mathrm{C} & 4.51410 \\ \mathrm{H} & 4.96597 \\ \mathrm{H} & 3.74897 \\ \mathrm{H} & 5.54484 \\ \mathrm{C} & 0.34940 \\ \mathrm{H} & -0.46640 \\ \mathrm{H} & 1.35194 \\ \mathrm{C} & 0.02873 \\ \mathrm{O} & -1.09887 \\ \mathrm{O} & 1.14026 \\ \mathrm{C} & 0.90745 \\ \mathrm{H} & 1.88824 \\ \mathrm{H} & 0.46443 \\ \mathrm{H} & 0.23820 \\ \mathrm{H}=-897.2331759 .4 .\end{array}$

$-0.02383$

$-1.35896$

$-1.70926$

0.24204

$-0.01656$

0.66911

1.25732

$-0.00310$

$-0.72146$

$-2.69775$

$-0.77696$

$-0.52154$

$-0.91039$

0.03791

$-2.27928$

$-0.37840$

1.09423

$-2.68409$

$-3.01175$

$-1.73689$

0.36370

$-3.74204$

$-2.05523$

1.24447

0.83783

1.15549

2.35702

2.66007

3.04381

4.14812

4.58847

3.80298

4.87890
$-0.62186$

$-0.06239$

0.25825

$-1.10102$

$-1.42405$

0.16914

$-0.89367$

$-2.15983$

$-0.26062$

0.86743

$-0.15229$

0.13563

$-0.02395$

$-0.21684$

0.01206

$-0.38649$

$-0.23738$

$-0.14617$

0.16927

$-0.34946$

$-0.54263$

$-0.11178$

$-0.47449$

1.56360

2.14606

1.96286

0.69656

0.30593

0.29255

$-0.59527$

$-0.77607$

$-1.53371$

$-0.13407$

Imaginary frequency $=i 402 \mathrm{~cm}^{-1}$ 
$-4.16548$

$-4.31089$

$-3.08533$

$-2.72404$

$-4.91750$

$-4.33948$

$-2.23365$

$-2.65073$

$-2.10317$

$-2.90864$

$-0.78595$

0.32459

1.65945

2. 84851

1.76145

4.09431

2.81529

3. 00233

0.85263

4.17883

4.99857

3.05381

5.14715

0.22540

$-0.76917$

0.96564

0.36896

$-0.38939$

1.43309

1.64486

2.53622

1.79916

0.78356

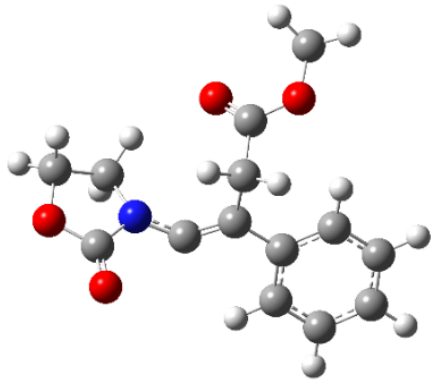

0.30244

$-0.84349$

$-1.28691$

0.22024

0.22705

1.20614

1.18966

$-0.32919$

$-0.56232$

$-2.15024$

$-0.92735$

$-0.34879$

$-0.96252$

$-0.23770$

$-2.30480$

$-0.83048$

0.79819

$-2.89314$

$-2.88430$

$-2.15806$

$-0.24837$

$-3.93151$

$-2.61902$

0.95467

1.00545

0.96784

2.20368

2. 52079

2. 94850

4.16395

4.61695

3.93398

4.82758
$-0.72983$

0.13842

0.53885

$-1.24372$

$-1.51606$

$-0.13659$

$-1.34117$

$-2.19043$

$-0.15992$

1.36015

$-0.16499$

0.29082

0.06639

0.25996

$-0.35030$

0.03286

0.58024

$-0.57654$

$-0.48043$

$-0.38696$

0.18675

$-0.89161$

$-0.55852$

1.09246

1.54529

1.89469

0.23676

$-0.66021$

0.59961

$-0.15009$

0.28209

$-1.20666$

$-0.04599$

$H=-897.276903$ a.u.

\section{TS1}

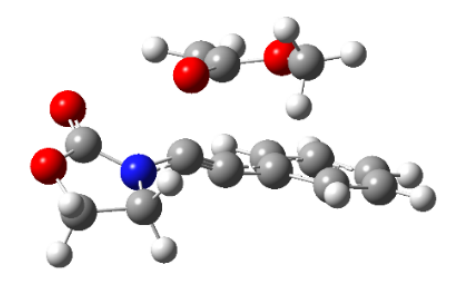




$\begin{array}{lrrr}\mathrm{C} & -3.63091 & -1.33018 & 1.45993 \\ \mathrm{O} & -3.73490 & -1.68072 & 0.06351 \\ \mathrm{C} & -2.52511 & -1.58463 & -0.54641 \\ \mathrm{C} & -2.27057 & -0.62264 & 1.60269 \\ \mathrm{H} & -4.47575 & -0.68704 & 1.70869 \\ \mathrm{H} & -3.68221 & -2.25361 & 2.04497 \\ \mathrm{H} & -1.71593 & -0.95326 & 2.48469 \\ \mathrm{H} & -2.36418 & 0.46670 & 1.60117 \\ \mathrm{~N} & -1.60760 & -1.07516 & 0.37304 \\ \mathrm{O} & -2.31068 & -1.90305 & -1.69072 \\ \mathrm{C} & -0.32867 & -0.74604 & 0.04594 \\ \mathrm{C} & 0.89700 & -0.82472 & 0.22357 \\ \mathrm{C} & 2.30515 & -0.69853 & 0.19980 \\ \mathrm{C} & 2.96857 & 0.22130 & 1.04631 \\ \mathrm{C} & 3.07815 & -1.51123 & -0.66414 \\ \mathrm{C} & 4.35664 & 0.32066 & 1.02370 \\ \mathrm{H} & 2.38229 & 0.85046 & 1.70811 \\ \mathrm{C} & 4.46496 & -1.39471 & -0.68268 \\ \mathrm{H} & 2.57505 & -2.22246 & -1.31117 \\ \mathrm{C} & 5.11086 & -0.48287 & 0.16038 \\ \mathrm{H} & 4.85370 & 1.03131 & 1.67748 \\ \mathrm{H} & 5.04585 & -2.01973 & -1.35451 \\ \mathrm{H} & 6.19329 & -0.39880 & 0.14473 \\ \mathrm{C} & -0.54941 & 0.86895 & -1.57308 \\ \mathrm{H} & -1.24095 & 0.23673 & -2.11575 \\ \mathrm{H} & 0.46794 & 0.95062 & -1.93359 \\ \mathrm{C} & -1.09322 & 1.94776 & -0.77446 \\ \mathrm{O} & -2.24564 & 2.01145 & -0.34933 \\ \mathrm{O} & -0.16129 & 2.90468 & -0.49190 \\ \mathrm{C} & -0.61821 & 4.00589 & 0.30672 \\ \mathrm{H} & -0.93742 & 3.66241 & 1.29514 \\ \mathrm{H} & 0.23668 & 4.67667 & 0.39461 \\ \mathrm{H} & -1.45644 & 4.51327 & -0.17794 \\ & & & \end{array}$

$H=-897.232666$ a.u.

Imaginary frequency $=i 489 \mathrm{~cm}^{-1}$

$\mathbf{I}^{\bullet}$

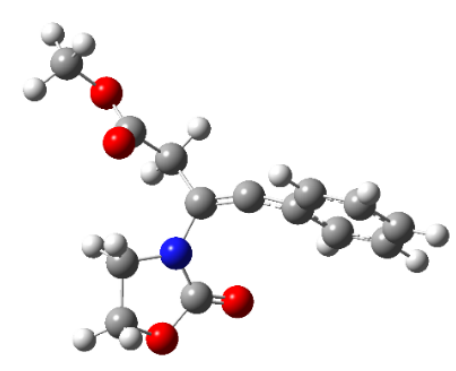

$\begin{array}{rrrr}\mathrm{C} & 1.23666 & 3.19099 & 1.20757 \\ \mathrm{O} & 0.31773 & 3.51655 & 0.14524 \\ \mathrm{C} & -0.12435 & 2.36243 & -0.44888 \\ \mathrm{C} & 1.71459 & 1.76693 & 0.88970 \\ \mathrm{H} & 2.03623 & 3.93318 & 1.20542 \\ \mathrm{H} & 0.69501 & 3.23620 & 2.15863\end{array}$




$\begin{array}{rrrr}\mathrm{H} & 1.83066 & 1.14262 & 1.77700 \\ \mathrm{H} & 2.66543 & 1.76784 & 0.33797 \\ \mathrm{~N} & 0.61089 & 1.30196 & 0.05074 \\ \mathrm{O} & -1.00559 & 2.34229 & -1.27739 \\ \mathrm{C} & 0.44101 & -0.04193 & -0.41850 \\ \mathrm{C} & -0.72695 & -0.63697 & -0.32804 \\ \mathrm{C} & -2.07795 & -0.84276 & -0.08410 \\ \mathrm{C} & -2.50949 & -1.61270 & 1.03996 \\ \mathrm{C} & -3.07057 & -0.33606 & -0.97953 \\ \mathrm{C} & -3.85973 & -1.85374 & 1.24986 \\ \mathrm{H} & -1.76357 & -2.00510 & 1.72389 \\ \mathrm{C} & -4.41456 & -0.59268 & -0.74988 \\ \mathrm{H} & -2.75037 & 0.27615 & -1.81506 \\ \mathrm{C} & -4.82089 & -1.35183 & 0.35868 \\ \mathrm{H} & -4.17228 & -2.43700 & 2.11144 \\ \mathrm{H} & -5.15836 & -0.19184 & -1.43259 \\ \mathrm{H} & -5.87541 & -1.54687 & 0.52884 \\ \mathrm{C} & 1.63696 & -0.69392 & -1.08426 \\ \mathrm{H} & 2.14650 & 0.01392 & -1.75084 \\ \mathrm{H} & 1.29295 & -1.51659 & -1.71897 \\ \mathrm{C} & 2.67048 & -1.27034 & -0.12683 \\ \mathrm{O} & 2.62166 & -1.24205 & 1.08608 \\ \mathrm{O} & 3.67973 & -1.84268 & -0.81725 \\ \mathrm{C} & 4.72439 & -2.45012 & -0.02976 \\ \mathrm{H} & 4.31315 & -3.24337 & 0.59864 \\ \mathrm{H} & 5.43056 & -2.85710 & -0.75254 \\ \mathrm{H} & 5.20748 & -1.70239 & 0.60364 \\ & & & \end{array}$

$H=-897.280469$ a.u.

\section{$\operatorname{ld}(\mathrm{C} \alpha)^{\bullet}$ inversion transition state}

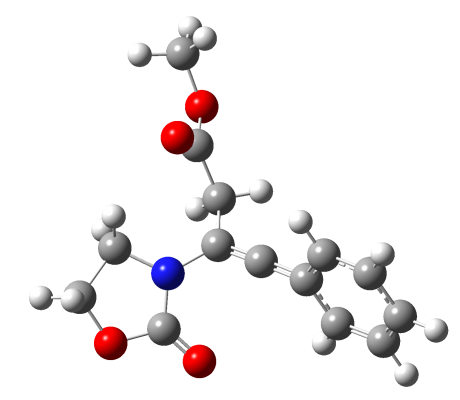

$\begin{array}{rrrr}\mathrm{C} & 2.068844 & 3.110599 & 1.002652 \\ \mathrm{O} & 1.029004 & 3.606689 & 0.136923 \\ \mathrm{C} & 0.277541 & 2.557913 & -0.328461 \\ \mathrm{C} & 2.207870 & 1.623850 & 0.641954 \\ \mathrm{H} & 2.972948 & 3.692405 & 0.817517 \\ \mathrm{H} & 1.748858 & 3.249175 & 2.040990 \\ \mathrm{H} & 2.367965 & 0.985464 & 1.512287 \\ \mathrm{H} & 3.023796 & 1.451881 & -0.074764 \\ \mathrm{~N} & 0.902126 & 1.376209 & 0.033770 \\ \mathrm{O} & -0.743768 & 2.705444 & -0.958215 \\ \mathrm{C} & 0.401394 & 0.091772 & -0.348511 \\ \mathrm{C} & -0.859539 & -0.218498 & -0.175403 \\ \mathrm{C} & -2.192746 & -0.514974 & -0.012417 \\ \mathrm{C} & -2.647559 & -1.288539 & 1.106058\end{array}$




$\begin{array}{lrrr}\mathrm{C} & -3.169550 & -0.062089 & -0.959840 \\ \mathrm{C} & -3.992202 & -1.593707 & 1.248350 \\ \mathrm{C} & -4.507511 & -0.382528 & -0.793326 \\ \mathrm{C} & -4.932512 & -1.149865 & 0.304034 \\ \mathrm{C} & 1.375238 & -0.838346 & -1.052499 \\ \mathrm{H} & 1.985798 & -0.287741 & -1.780209 \\ \mathrm{H} & 0.812716 & -1.579048 & -1.628888 \\ \mathrm{C} & 2.314289 & -1.605288 & -0.131987 \\ \mathrm{O} & 2.372240 & -1.511320 & 1.077094 \\ \mathrm{O} & 3.098220 & -2.435117 & -0.852406 \\ \mathrm{C} & 4.030381 & -3.240880 & -0.101981 \\ \mathrm{H} & 3.495760 & -3.882655 & 0.601952 \\ \mathrm{H} & 4.558079 & -3.837923 & -0.844751 \\ \mathrm{H} & 4.727083 & -2.603951 & 0.448050 \\ \mathrm{H} & -2.839529 & 0.552334 & -1.790262 \\ \mathrm{H} & -5.234879 & -0.027384 & -1.517909 \\ \mathrm{H} & -5.983399 & -1.394186 & 0.424867 \\ \mathrm{H} & -4.319088 & -2.182256 & 2.101079 \\ \mathrm{H} & -1.920187 & -1.631678 & 1.834758\end{array}$

$\mathrm{H}=-897.281096$ a.u.

Imaginary frequency $=i 8 \mathrm{~cm}^{-1}$

\section{$\operatorname{ld}(C \beta)^{\bullet}$ inversion transition state}

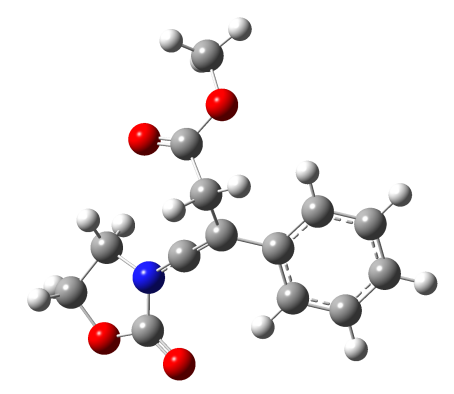

$\begin{array}{llll}\mathrm{C} & -3.679304 & -2.208264 & -1.965105 \\ \mathrm{O} & -3.384065 & -3.197175 & -0.953652 \\ \mathrm{C} & -2.408152 & -2.737933 & -0.119595 \\ \mathrm{C} & -2.444004 & -1.301742 & -1.994601 \\ \mathrm{H} & -3.863288 & -2.731333 & -2.904213 \\ \mathrm{H} & -4.580310 & -1.665490 & -1.661015 \\ \mathrm{H} & -2.673736 & -0.246595 & -2.148364 \\ \mathrm{H} & -1.703670 & -1.631580 & -2.733934 \\ \mathrm{~N} & -1.925062 & -1.520781 & -0.632537 \\ \mathrm{O} & -2.049353 & -3.300584 & 0.883290 \\ \mathrm{C} & -0.785951 & -0.927346 & -0.164994 \\ \mathrm{C} & 0.324593 & -0.348793 & 0.290824 \\ \mathrm{C} & 1.659453 & -0.962525 & 0.066386 \\ \mathrm{C} & 2.848507 & -0.237697 & 0.259958 \\ \mathrm{C} & 1.761448 & -2.304799 & -0.350296 \\ \mathrm{C} & 4.094315 & -0.830484 & 0.032857 \\ \mathrm{H} & 2.815292 & 0.798188 & 0.580244 \\ \mathrm{C} & 3.002332 & -2.893140 & -0.576536 \\ \mathrm{H} & 0.852628 & -2.884296 & -0.480426\end{array}$




$\begin{array}{rrrr}\mathrm{C} & 4.178827 & -2.158060 & -0.386958 \\ \mathrm{H} & 4.998575 & -0.248369 & 0.186746 \\ \mathrm{H} & 3.053809 & -3.931507 & -0.891607 \\ \mathrm{H} & 5.147148 & -2.619022 & -0.558522 \\ \mathrm{C} & 0.225401 & 0.954673 & 1.092464 \\ \mathrm{H} & -0.769167 & 1.005455 & 1.545288 \\ \mathrm{H} & 0.965640 & 0.967839 & 1.894692 \\ \mathrm{C} & 0.368965 & 2.203676 & 0.236756 \\ \mathrm{O} & -0.389393 & 2.520787 & -0.660208 \\ \mathrm{O} & 1.433087 & 2.948500 & 0.599613 \\ \mathrm{C} & 1.644859 & 4.163952 & -0.150090 \\ \mathrm{H} & 2.536221 & 4.616946 & 0.282094 \\ \mathrm{H} & 1.799162 & 3.933979 & -1.206663 \\ \mathrm{H} & 0.783559 & 4.827577 & -0.045988\end{array}$

$\mathrm{H}=-897.26257$ a.u.

Imaginary frequency $=i 217 \mathrm{~cm}^{-1}$

\subsection{Crystal structure for $3 a b$}

\begin{tabular}{|c|c|}
\hline Compound & $3 a b$ \\
\hline Formula & $\mathrm{C}_{22} \mathrm{H}_{30} \mathrm{I}_{2} \mathrm{~N}_{4} \mathrm{O}_{4}$ \\
\hline $\mathrm{M}_{\mathrm{w}}$ & 668.30 \\
\hline Crystal system & triclinic \\
\hline Measurement temperature/ K & 295 \\
\hline Space group & $P-1$ \\
\hline a/ $\AA$ & $9.2857(2)$ \\
\hline $\mathrm{c} / \AA$ & $16.1028(4)$ \\
\hline$a /^{\circ}$ & $72.507(2)$ \\
\hline $\mathrm{b} /{ }^{\circ}$ & $76.823(2)$ \\
\hline $\mathrm{g} /{ }^{\circ}$ & $87.285(2)$ \\
\hline $\mathrm{V} / \AA^{3}$ & $1347.46(6)$ \\
\hline Z & 2 \\
\hline $\mathrm{Dc} / \mathrm{g} \cdot \mathrm{cm}^{-3}$ & 1.647 \\
\hline Crystal colour & colourless \\
\hline Crystal size $/ \mathrm{mm}^{3}$ & $0.22^{*} 0.18^{*} 0.08$ \\
\hline $\mathrm{m}(\mathrm{Mo}-\mathrm{Ka}) / \mathrm{mm}^{-1}$ & 2.366 \\
\hline $\mathrm{N}^{\circ}$ of refl. measured & 24901 \\
\hline $\mathrm{N}^{\circ}$ of unique refl. & 6247 \\
\hline $\mathrm{N}^{\circ}$ of observed refl. $\left[\mathrm{F}^{2}>4 \mathrm{sF}^{2}\right]$ & 4678 \\
\hline $\mathrm{N}^{\circ}$ parameters refined & 291 \\
\hline $\mathrm{R}_{1}\left[\mathrm{~F}^{2}>4 \mathrm{sF}^{2}\right]$ & 0.0481 \\
\hline$w R_{1}\left[F^{2}>4 s F^{2}\right]$ & $0.1143^{\mathrm{a}}$ \\
\hline $\mathrm{R}_{2}$ [all refl.] & 0.0675 \\
\hline $\mathrm{wR}_{2}$ [all refl.] & 0.1259 \\
\hline Goodness of fit [all refl.] & 1.047 \\
\hline \multicolumn{2}{|c|}{${ }^{a} w=1 /\left[\mathrm{s}^{2}\left(F^{2}\right)+(0.0497 P)^{2}+1.9108 P\right]$ where $P=\left(F o^{2}+2 F c^{2}\right) / 3$} \\
\hline
\end{tabular}

Table of the crystallographic data for $3 a b$ 
Recrystallisation of $\mathbf{3 a b}$ was achieved from dichloromethane and pentane. A suitable crystal was selected and mounted on a Rigaku Oxford Diffraction SuperNova diffractometer and measured at 295K. Using Olex2 [Dolomanov, O. V.; Bourhis, L. J.; Gildea, R. J.; Howard, J. A. K.; Puschmann, H. OLEX2: a complete structure solution, refinement and analysis program. J. Appl. Cryst., 2009, 42, 339-341.] the structure was solved with the ShelXT structure solution program using Intrinsic Phasing [Sheldrick, G. M. SHELXT - Integrated space-group and crystal-structure determination. ActaCryst., 2015, A71, 3-8] and refined with the ShelXL refinement package using Least Squares minimisation [Sheldrick, G. M. Crystal structure refinement with SHELXL. ActaCryst. 2015, C71, 3-8.].

All H-atoms were found experimentally and their coordinates and Uiso parameters were constraint to $1.5 \mathrm{Ueq}$ (parent atoms) for the methyls and to $1.2 \mathrm{Ueq}$ (parent atom) for the other carbons. Compound 3ab crystallized with 2 independent molecules in the asymmetric unit.

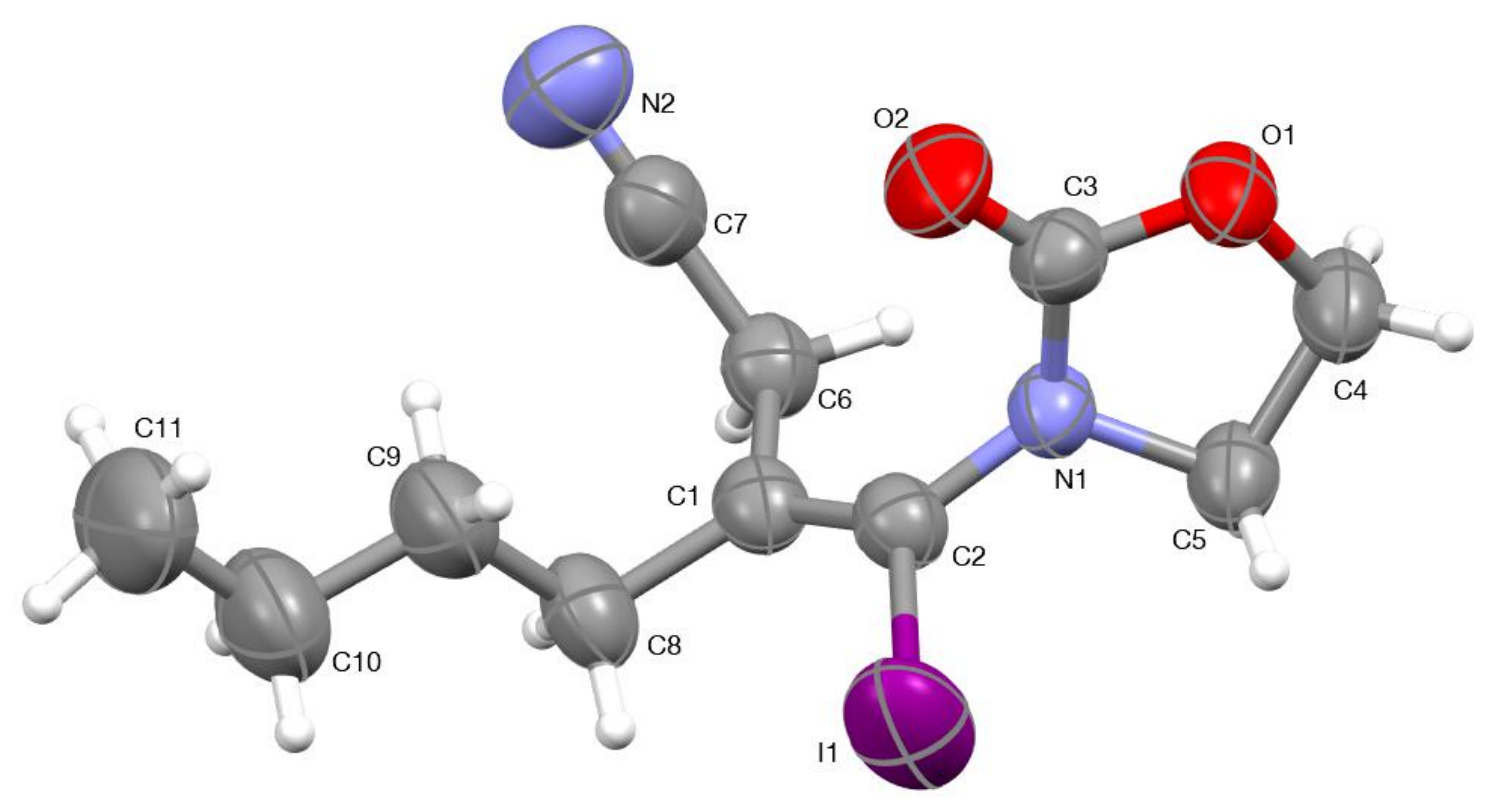

ORTEP view of the single crystal X-ray structure of compound 3ab drawn at 50\% probability level. Only one conformer has been represented for clarity.

\subsection{Addition of 2 a to $1 d$}


The relative ratio of isomers (determined from the ${ }^{1} \mathrm{H}$ NMR spectrum) was E:F:G:H = $3: 52: 18: 27$

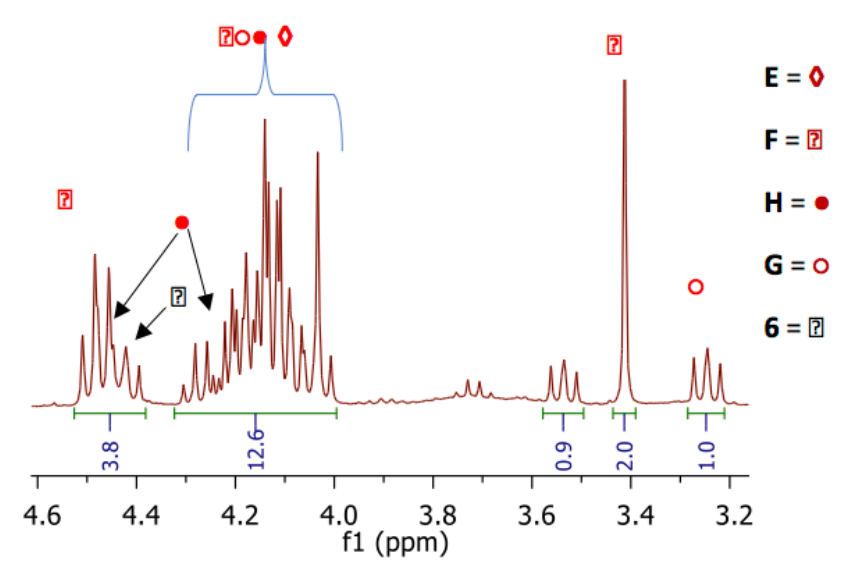

Characteristic signals of EFGH + hydroiodination product 6

\subsection{NMR spectra for all new compounds}




$$
\text { 3aa } \left.\sum_{0}^{N}\right\rangle=0
$$

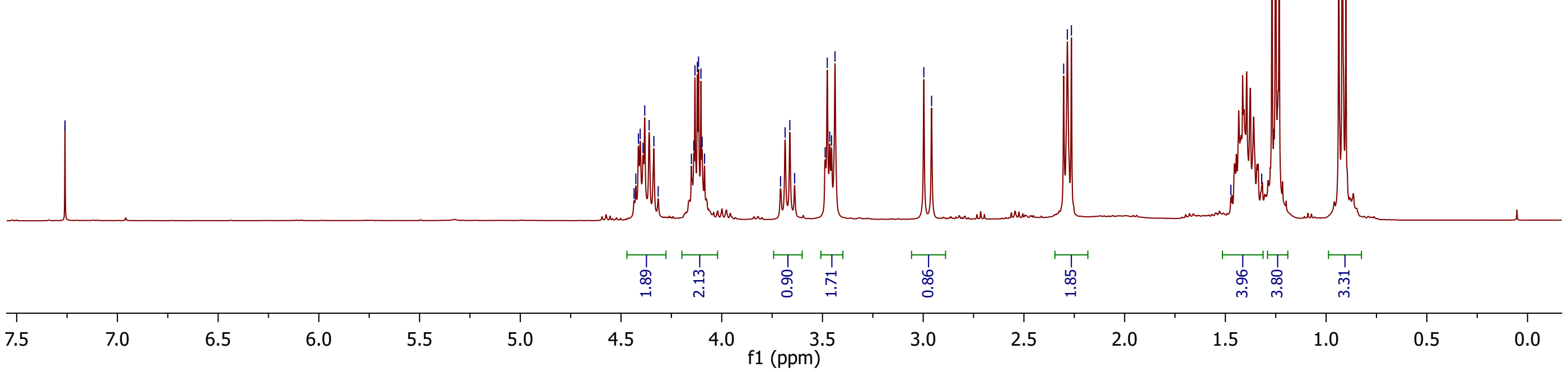




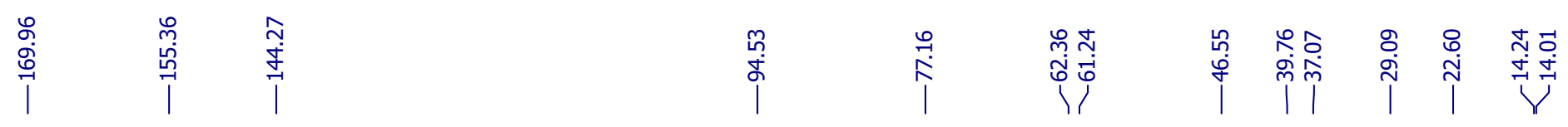

3aa $\left.\sum_{0}^{\mathrm{N}}\right\rangle=0$

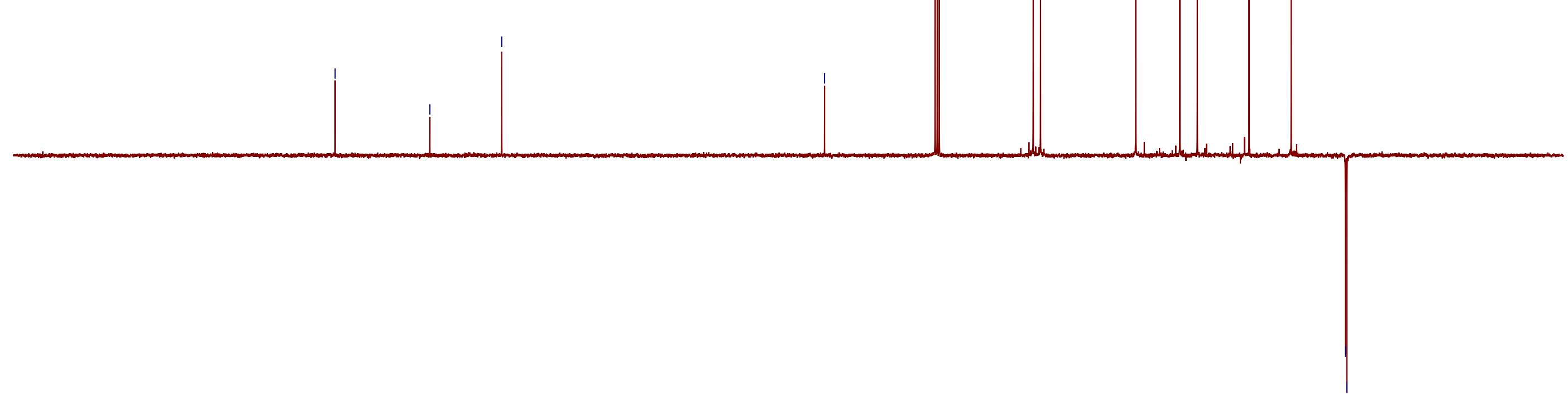

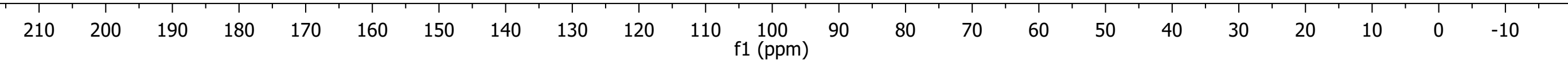



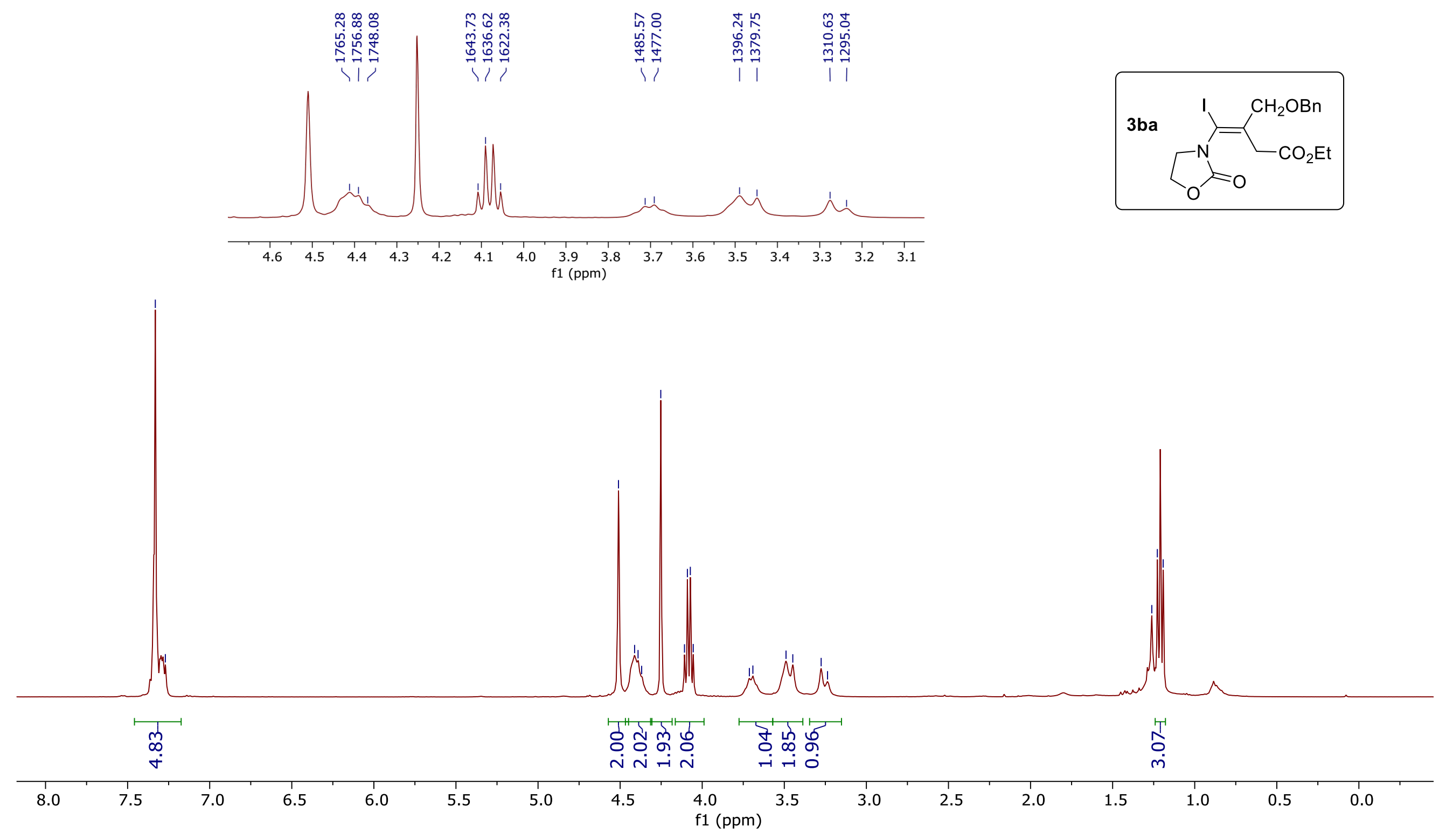


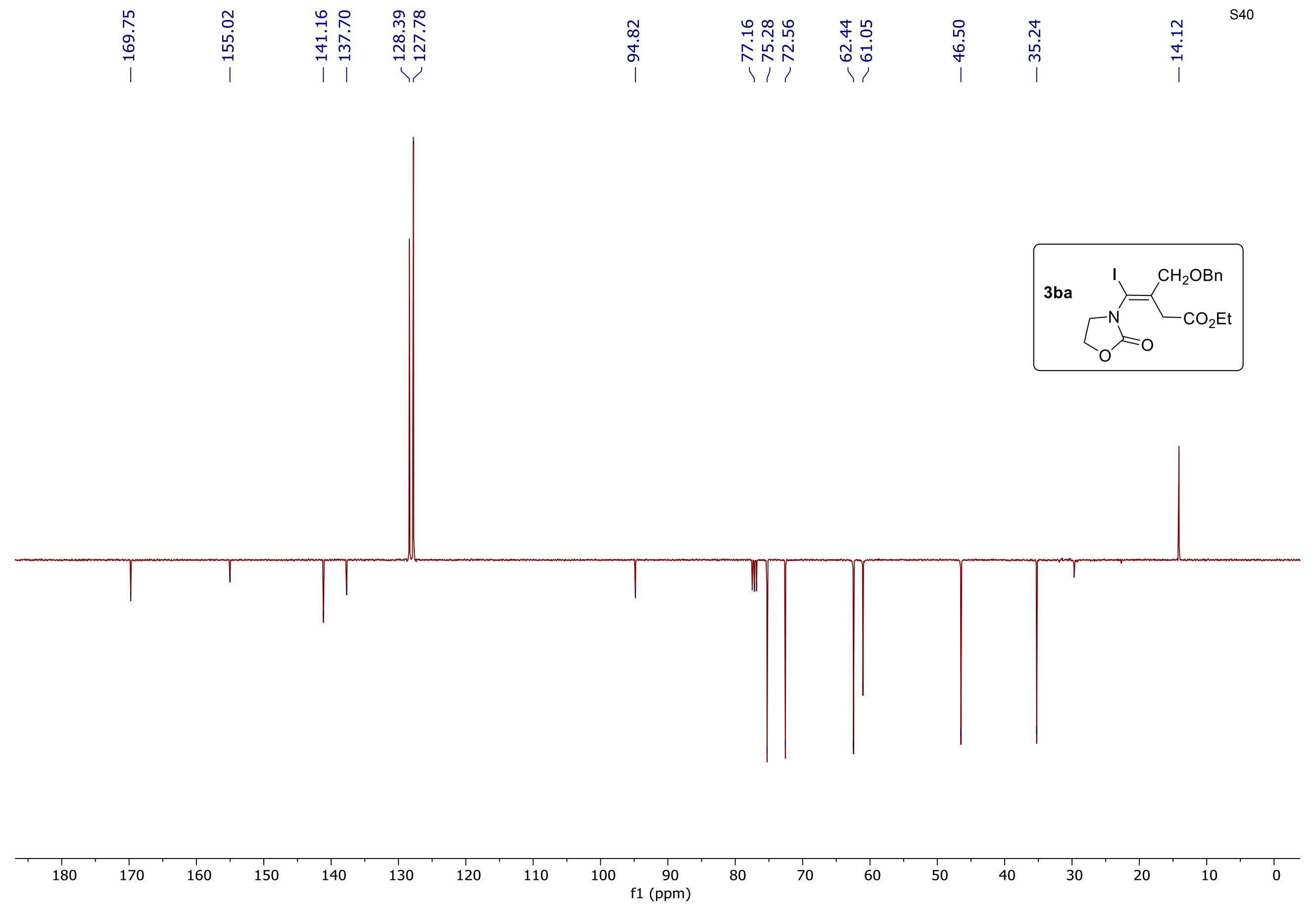



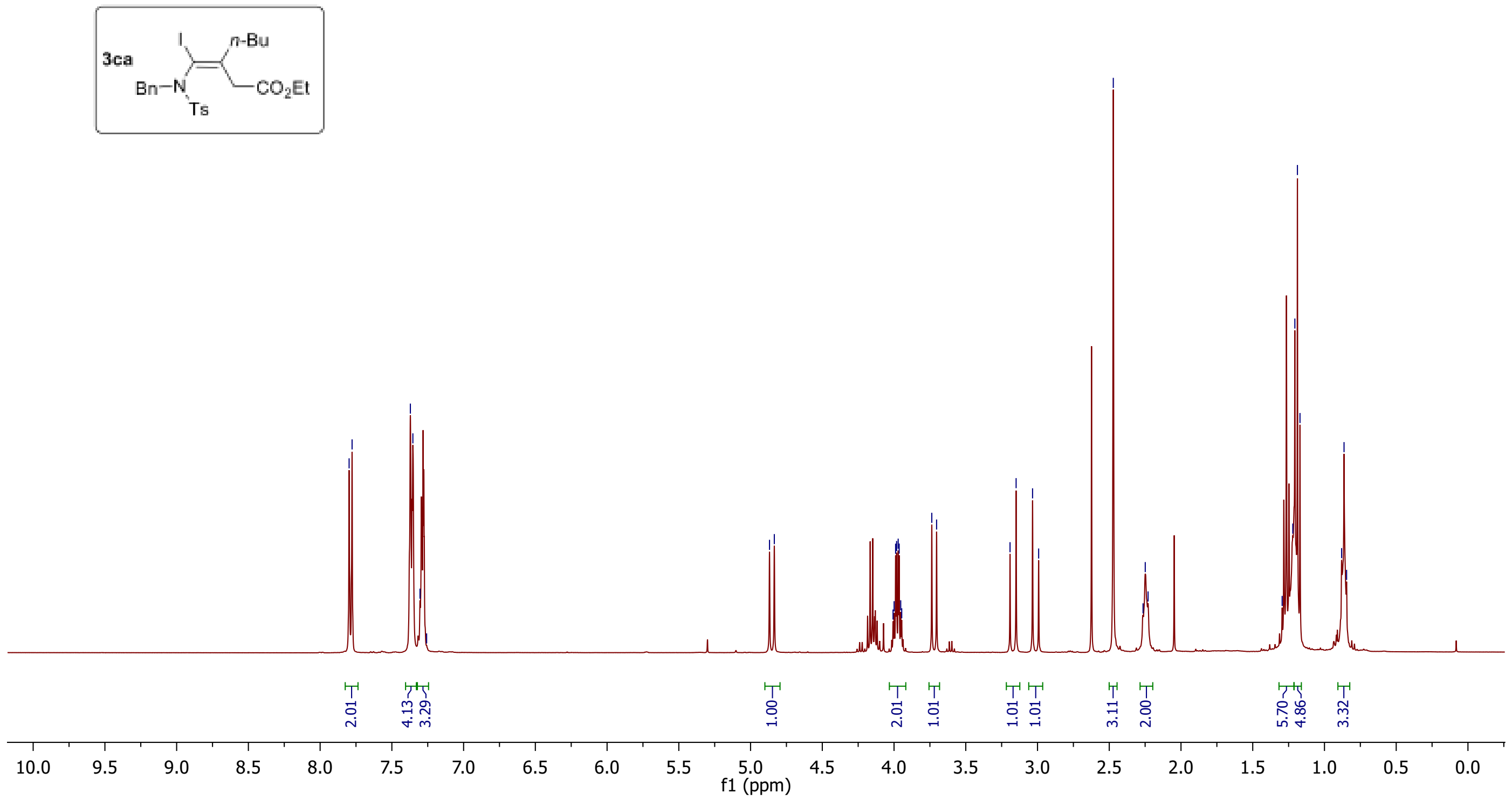

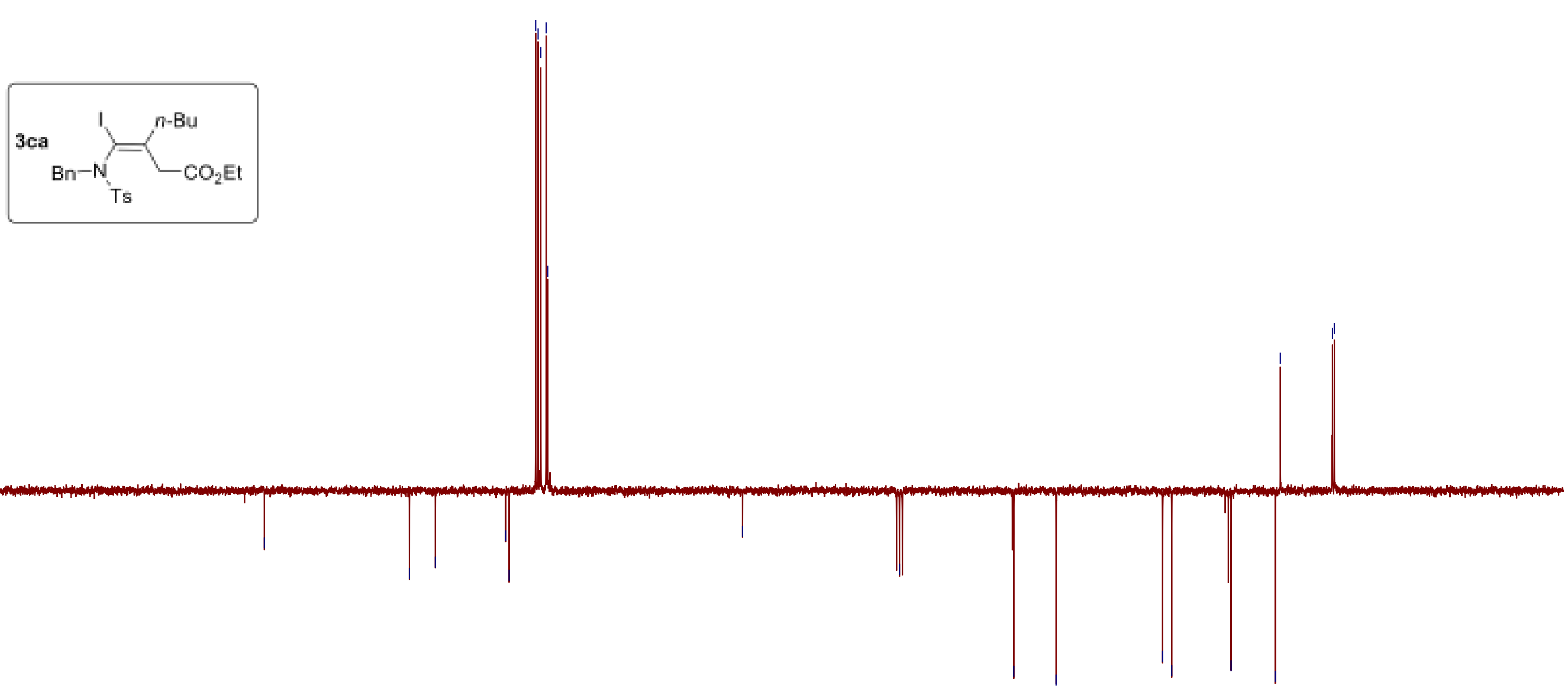

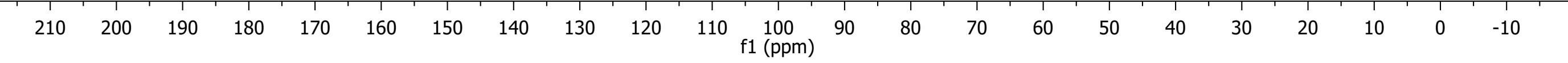



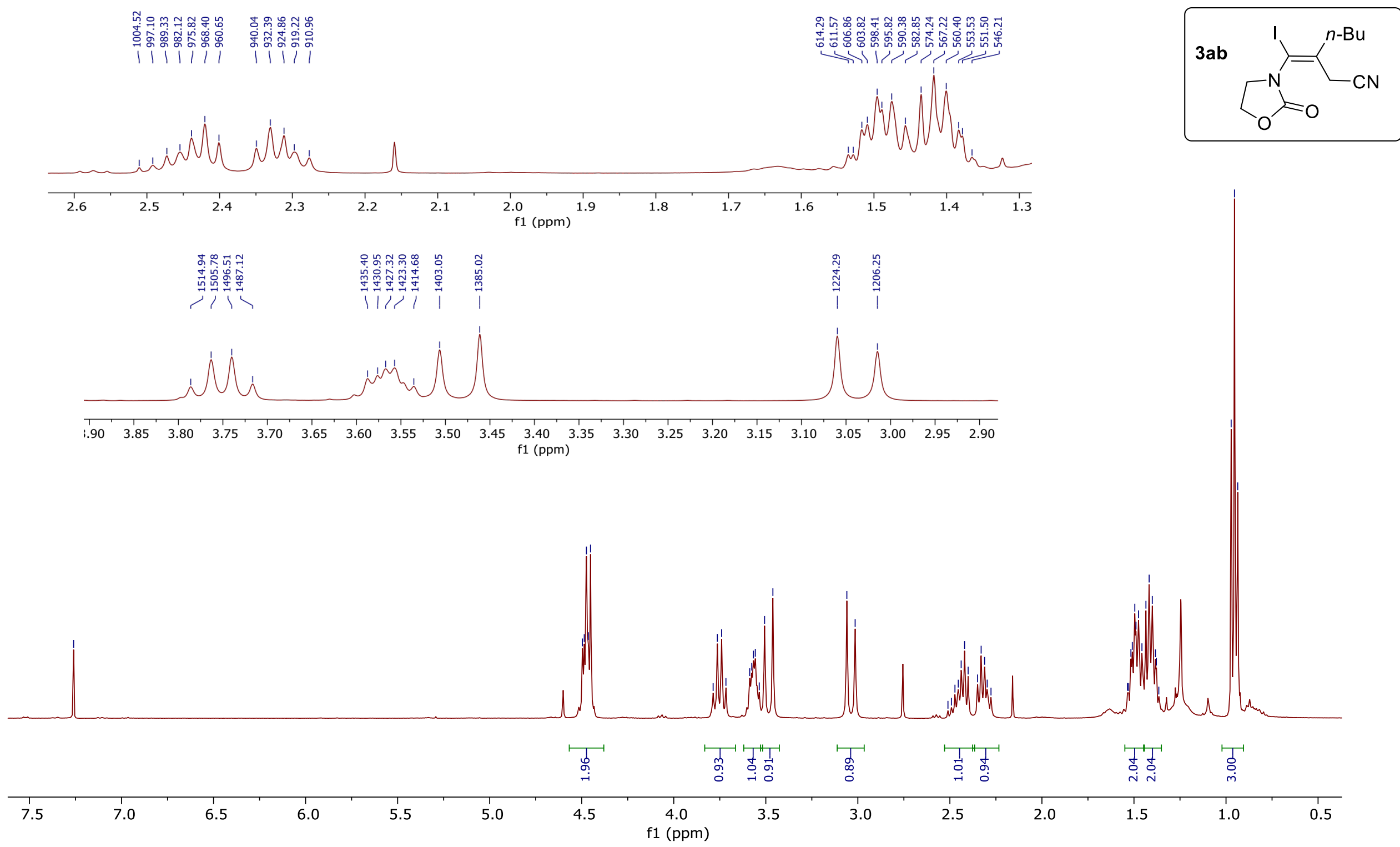


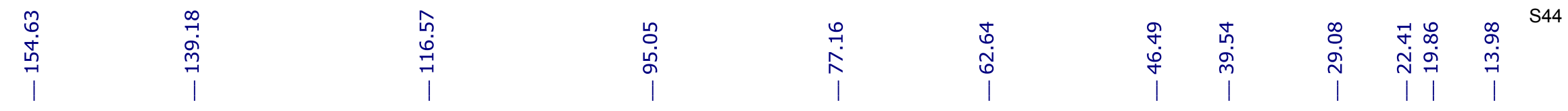

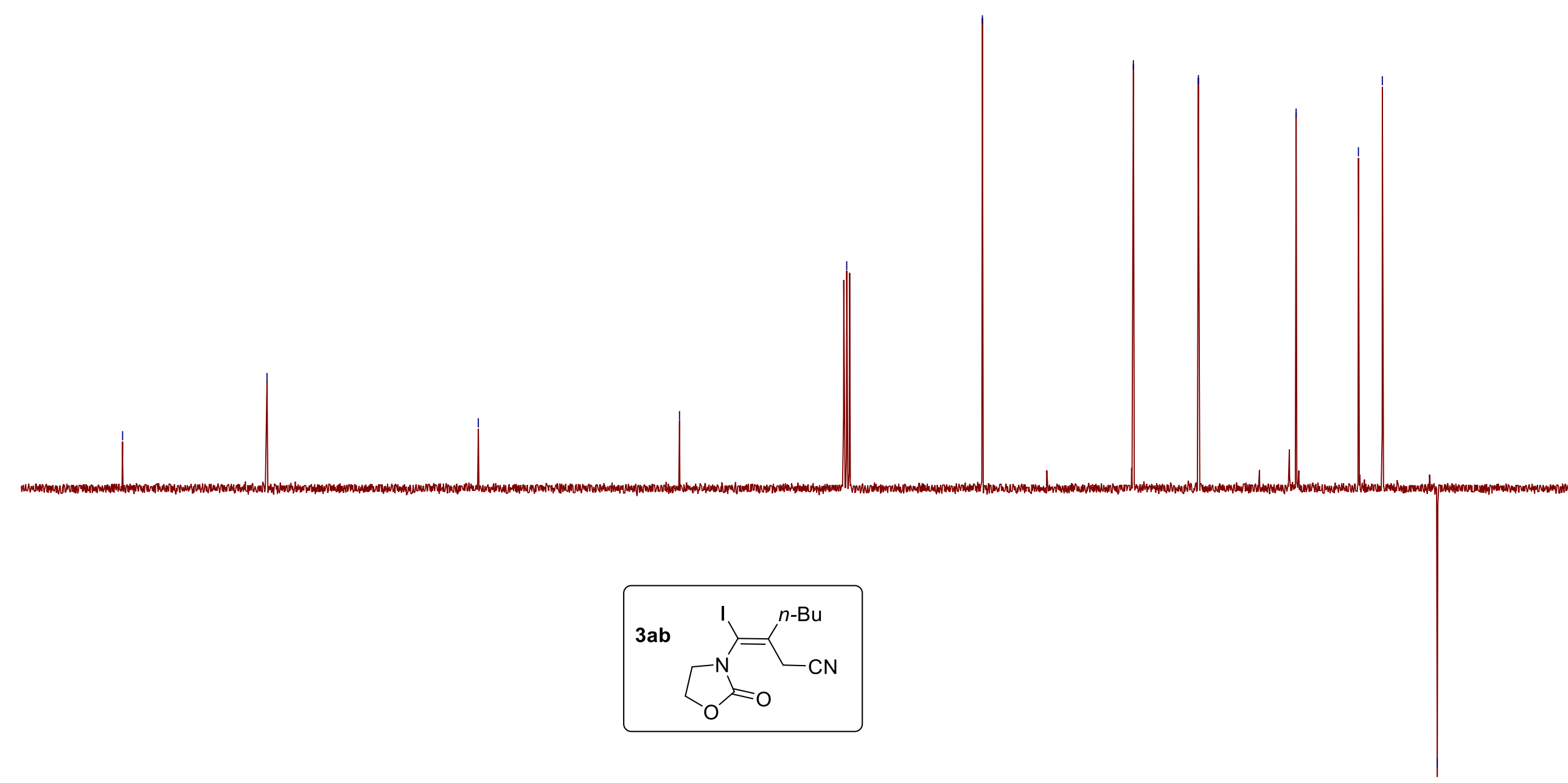

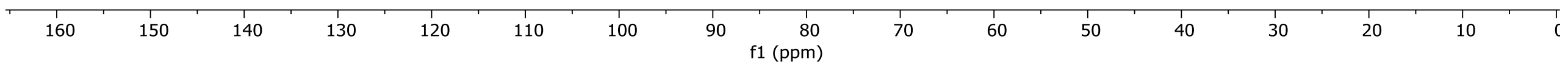




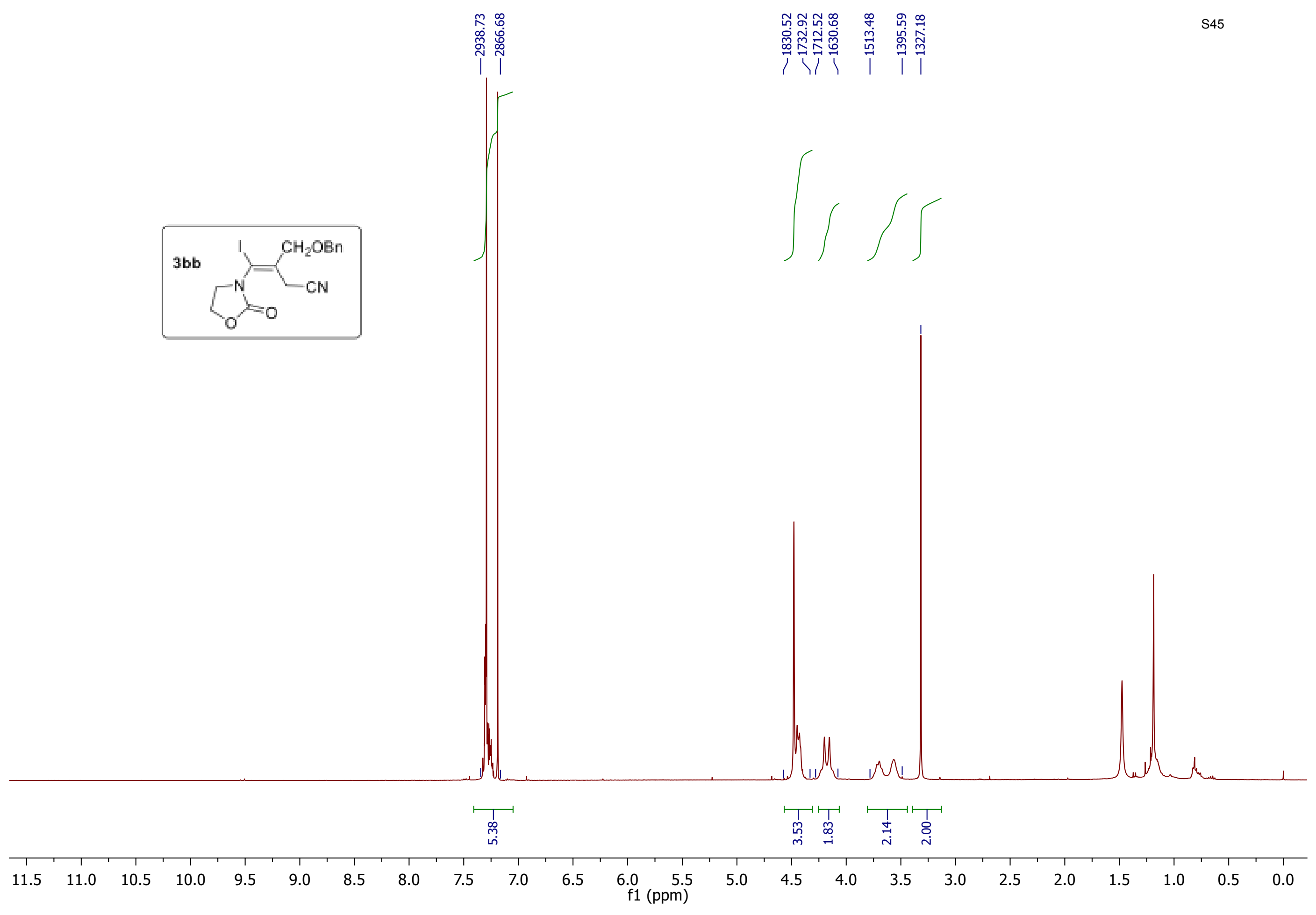




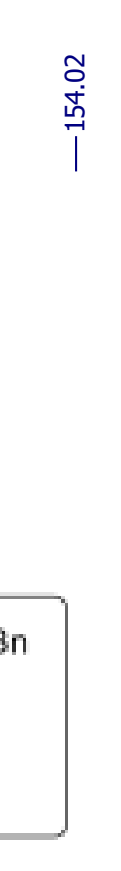
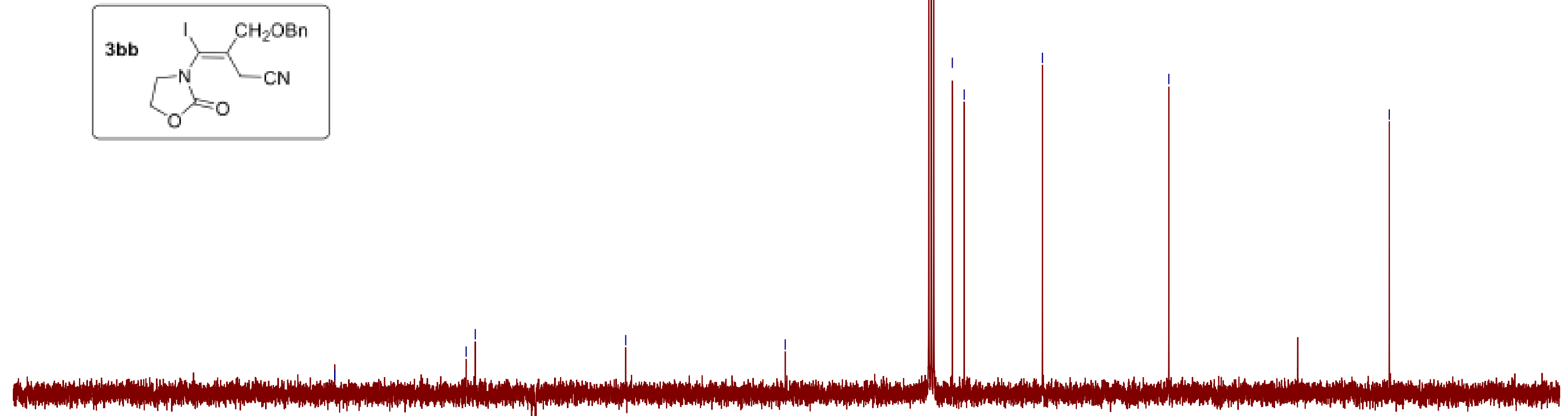

\begin{tabular}{|c|c|c|c|c|c|c|c|c|c|c|c|c|c|c|c|c|c|c|}
\hline 1 & 1 & $T$ & 1 & $T$ & $T$ & 1 & 1 & 1 & $T$ & 1 & 1 & $T$ & $T$ & 1 & $T$ & $T$ & $T$ & $T$ \\
\hline 190 & 180 & 170 & 160 & 150 & 140 & 130 & 120 & 110 & $\begin{array}{c}100(\mathrm{ppm}) \\
\mathrm{f} 1(\mathrm{pp}\end{array}$ & 80 & 70 & 60 & 50 & 40 & 30 & 20 & 10 & 0 \\
\hline
\end{tabular}




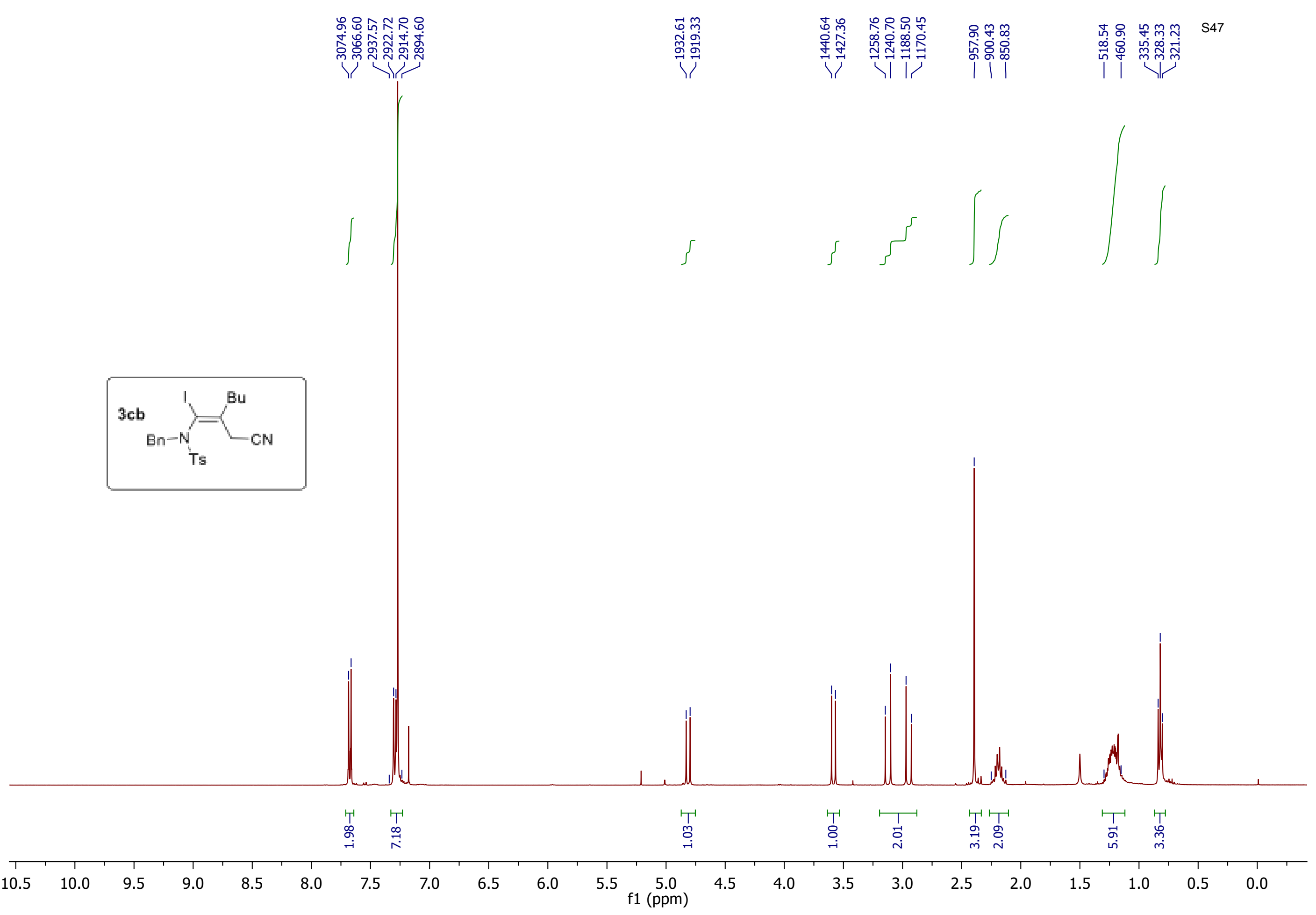




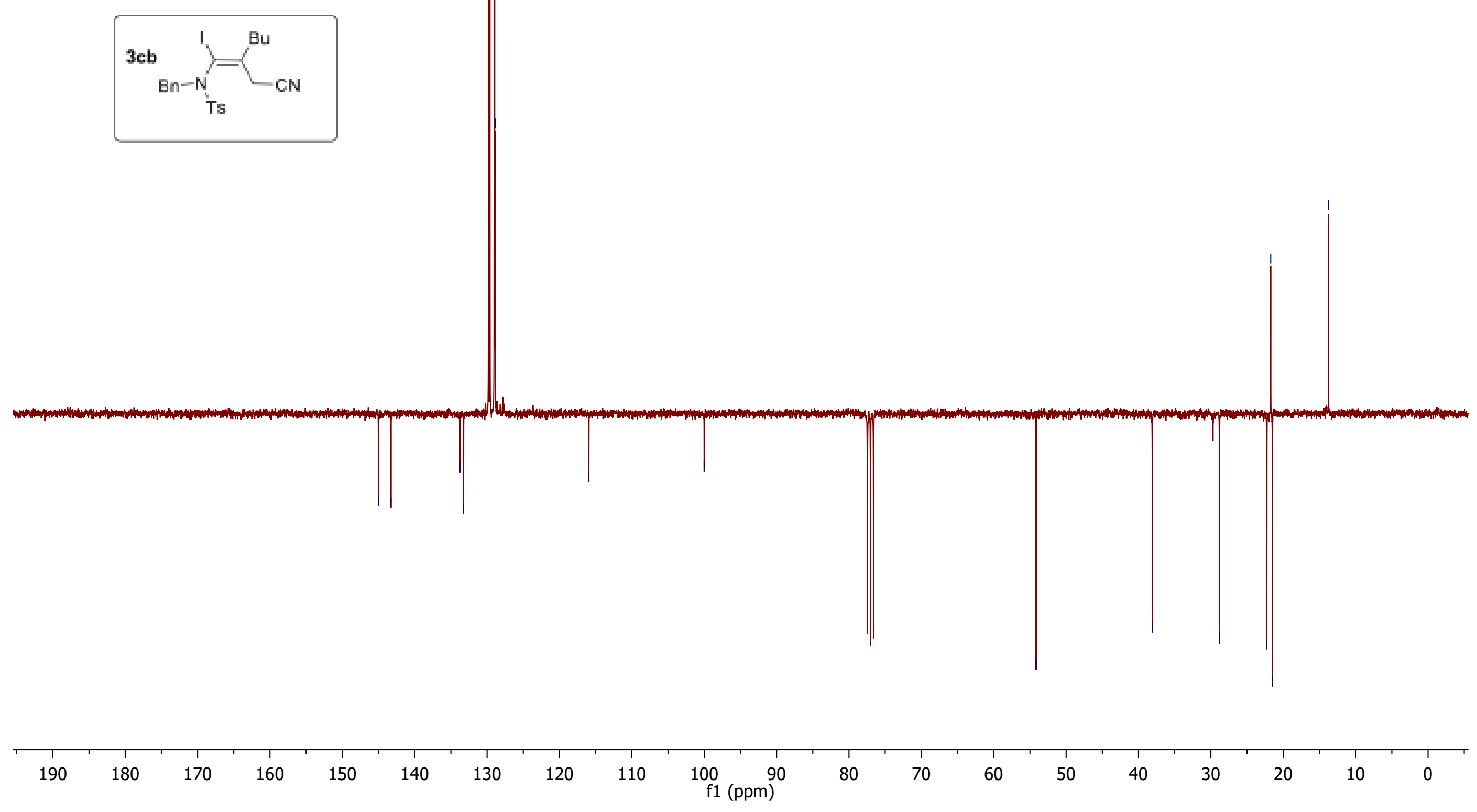



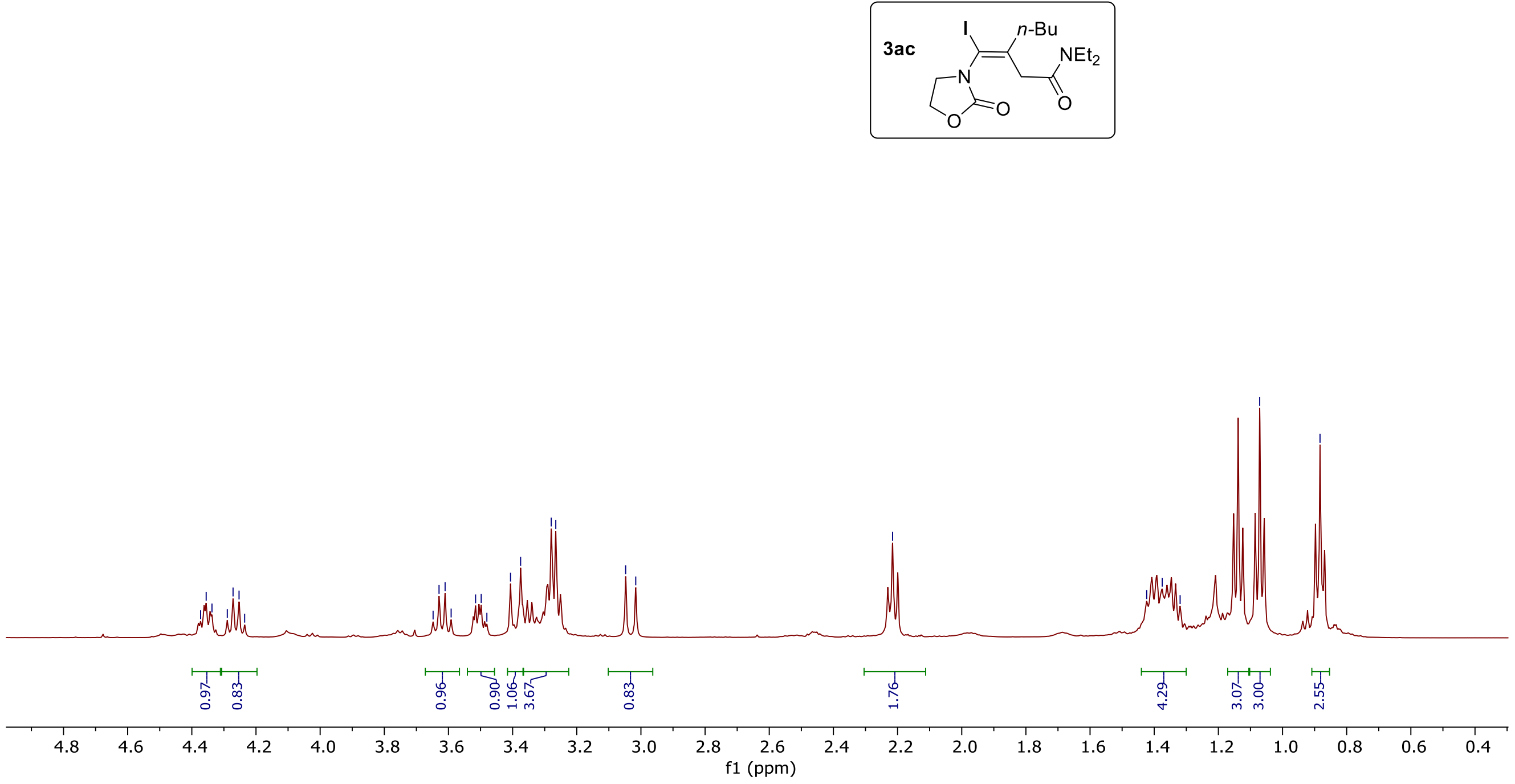


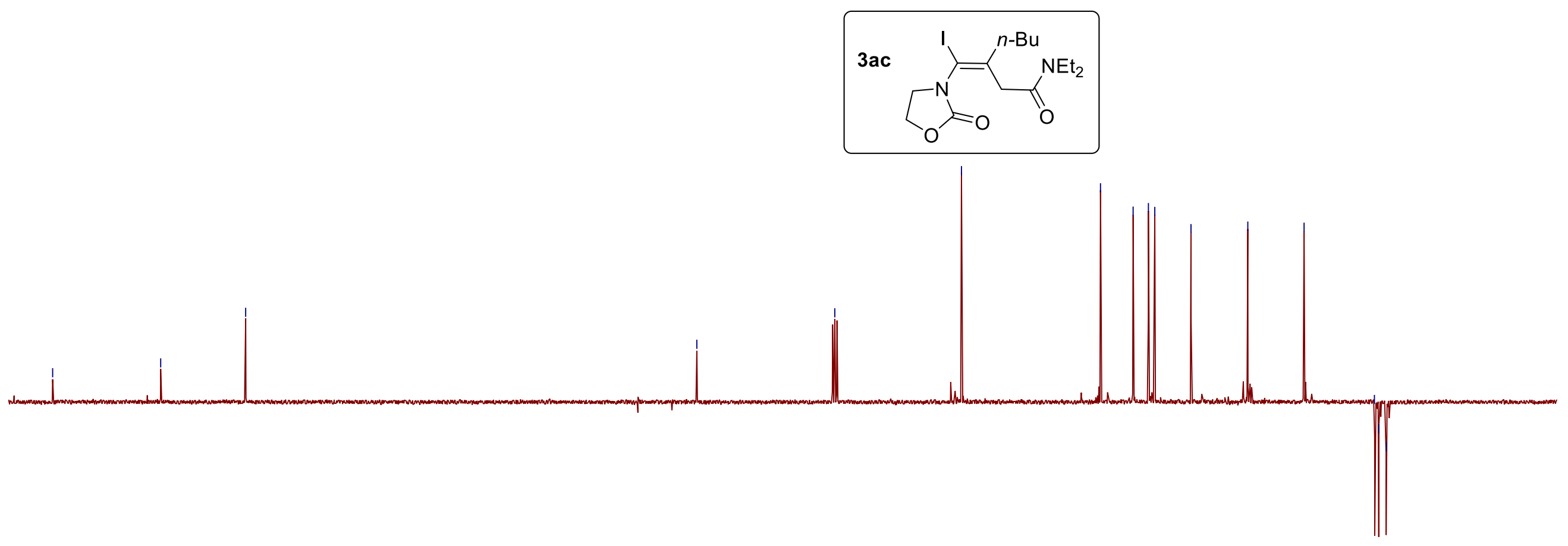

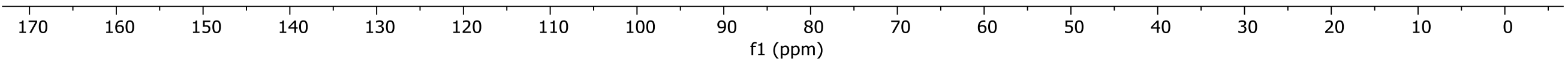



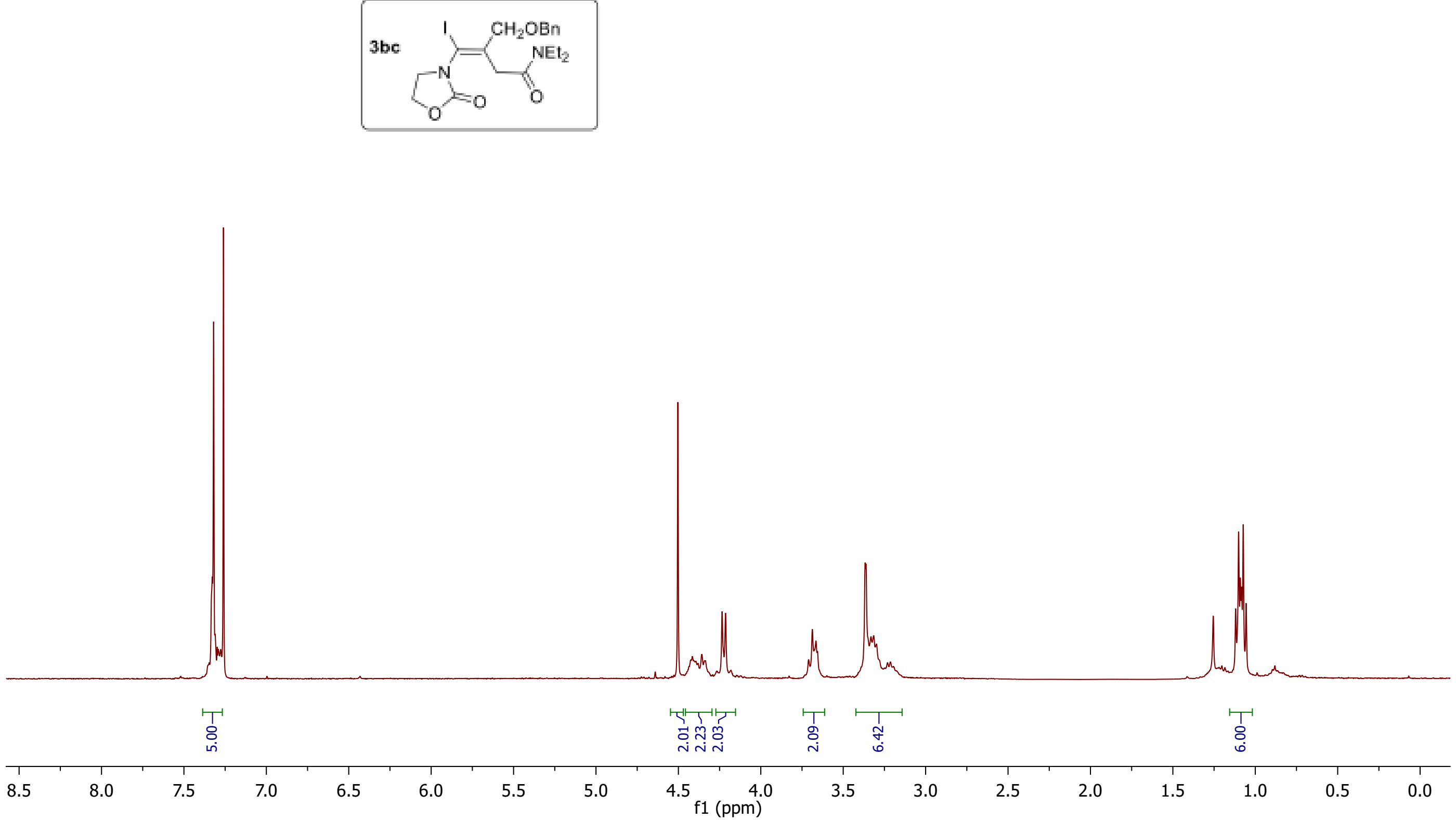


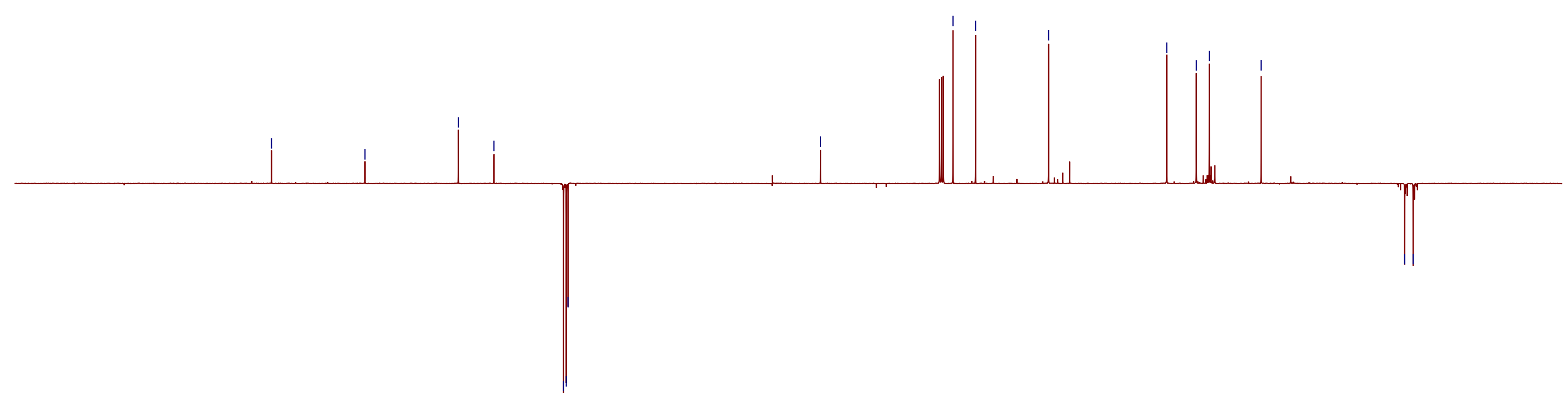

\begin{tabular}{|c|c|c|c|c|c|c|c|c|c|c|c|c|c|c|c|c|c|c|c|c|}
\hline 1 & 1 & 1 & 1 & 1 & 1 & 1 & 1 & 1 & 1 & 11 & 1 & 1 & 1 & 1 & 1 & 1 & $T$ & 1 & $T$ & $T$ \\
\hline 200 & 190 & 180 & 170 & 160 & 150 & 140 & 130 & 120 & 110 & $\begin{array}{l}100 \\
\text { f1 (ppm) }\end{array}$ & 90 & 80 & 70 & 60 & 50 & 40 & 30 & 20 & 10 & 0 \\
\hline
\end{tabular}



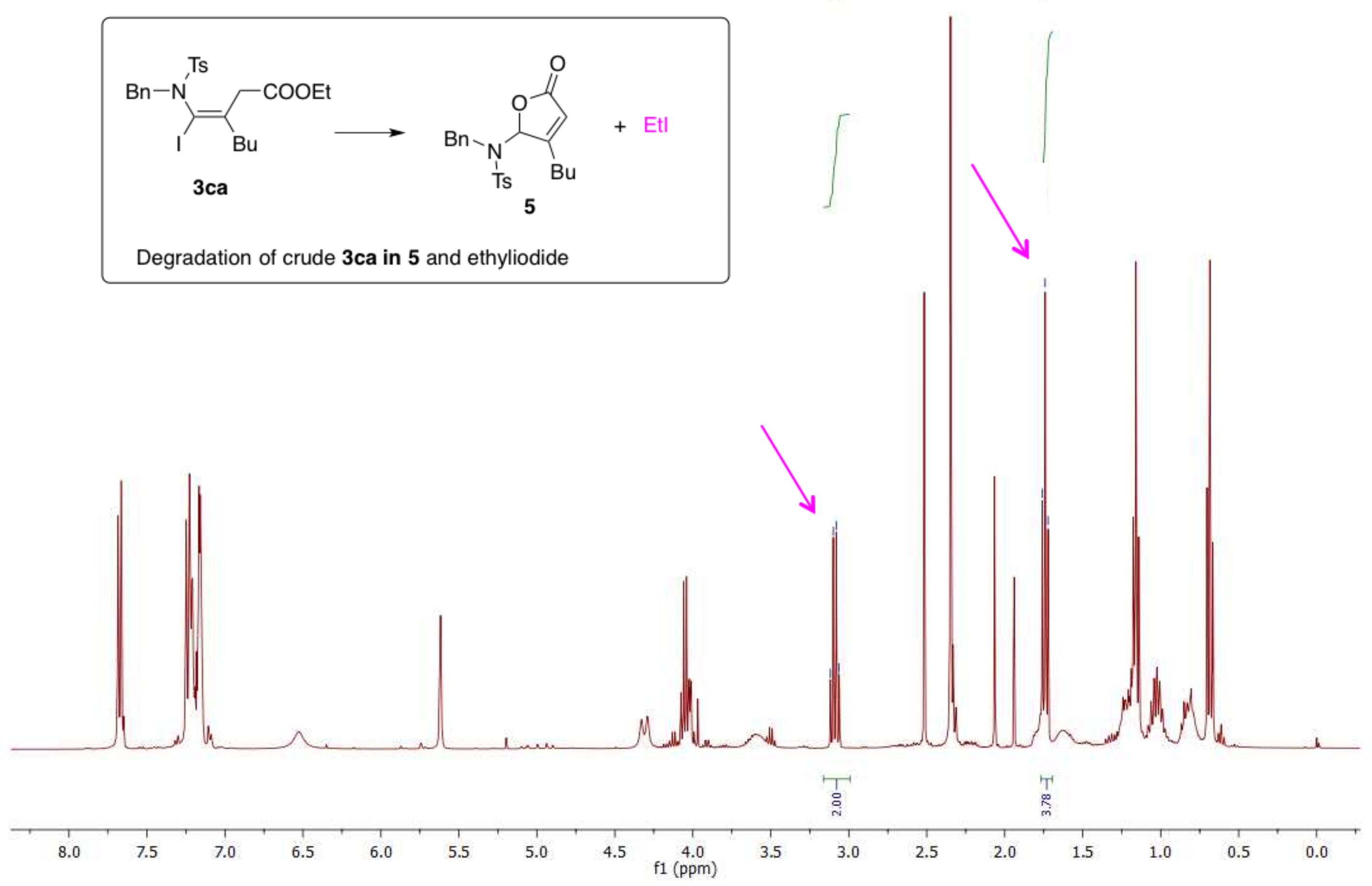

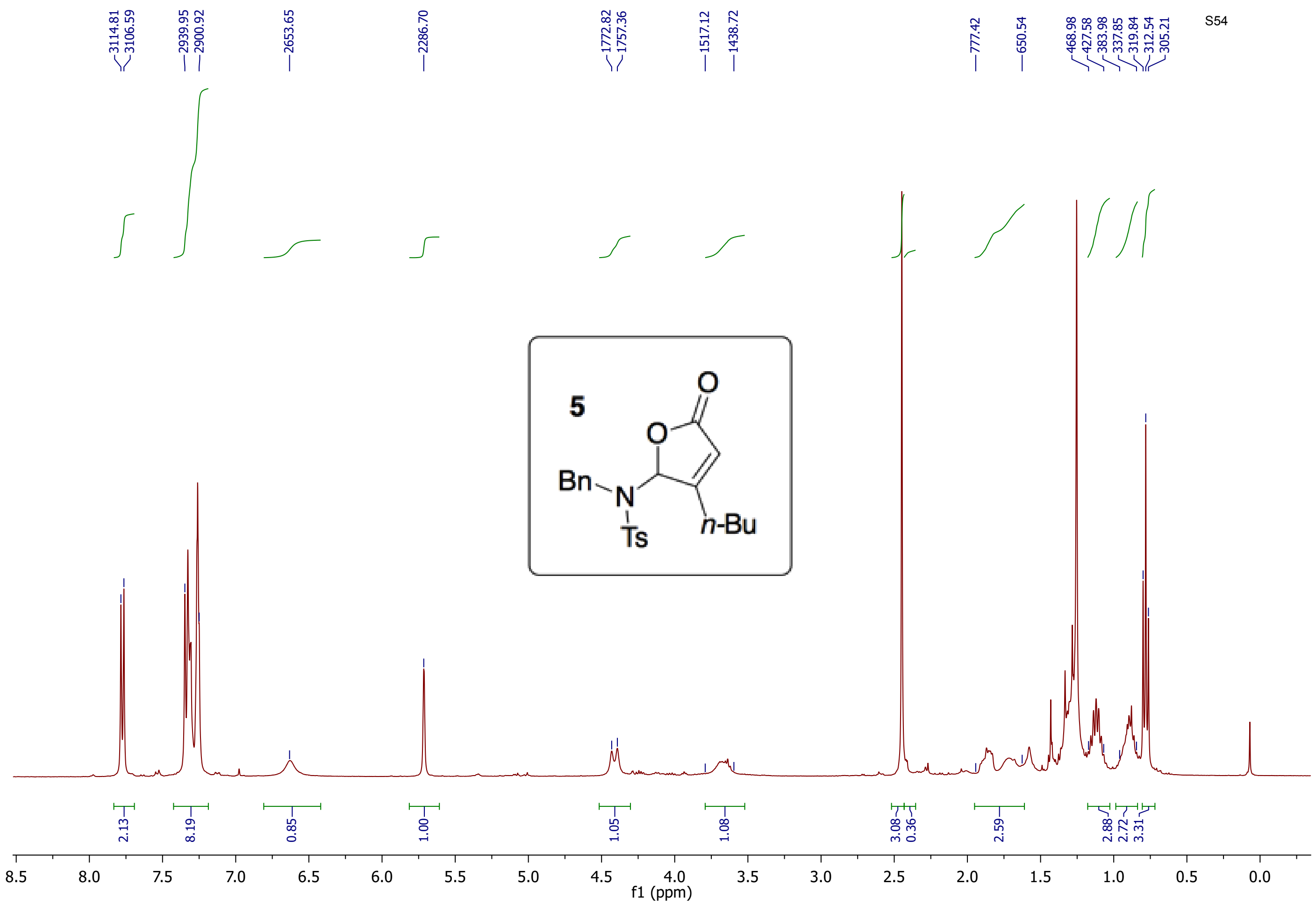


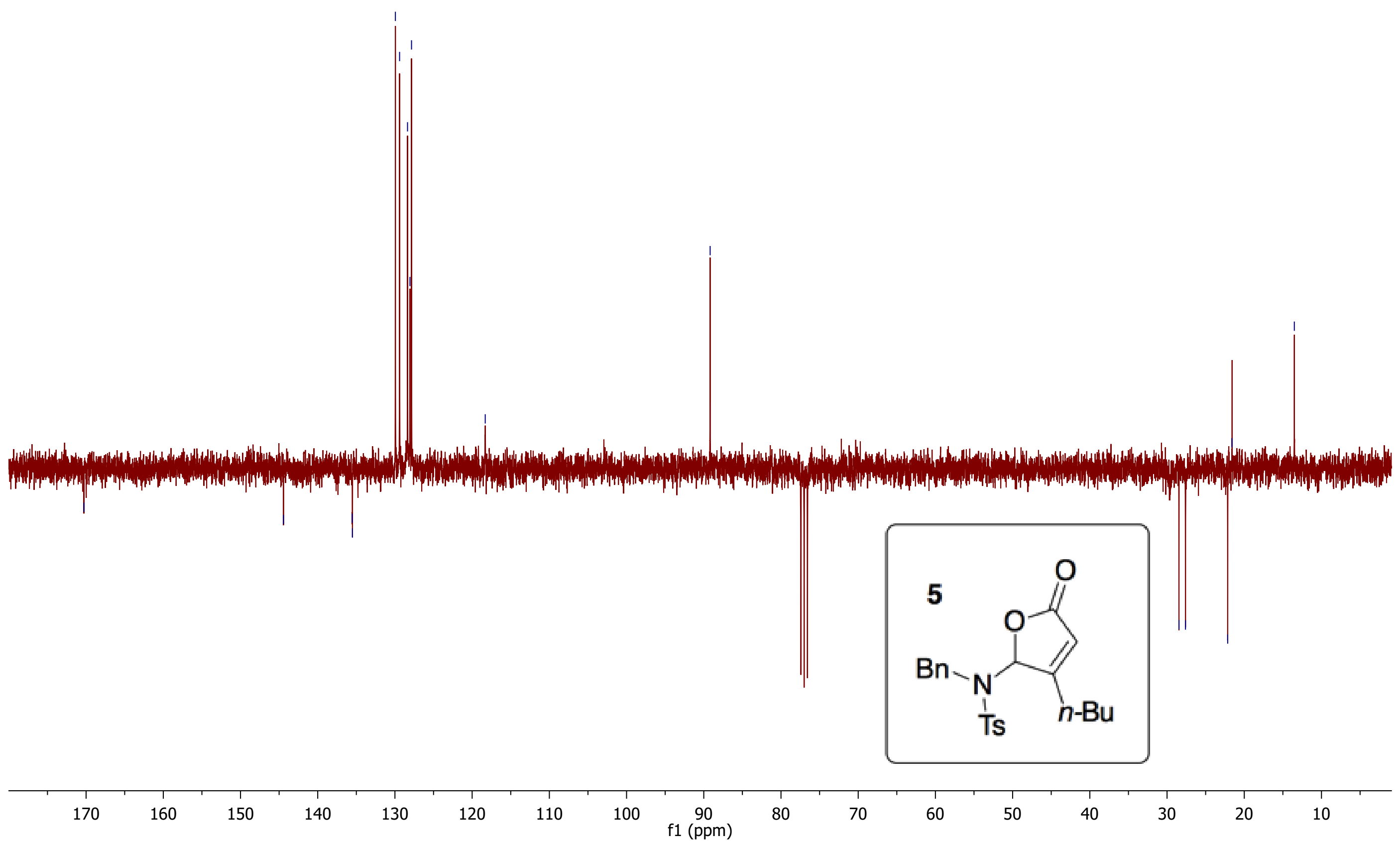




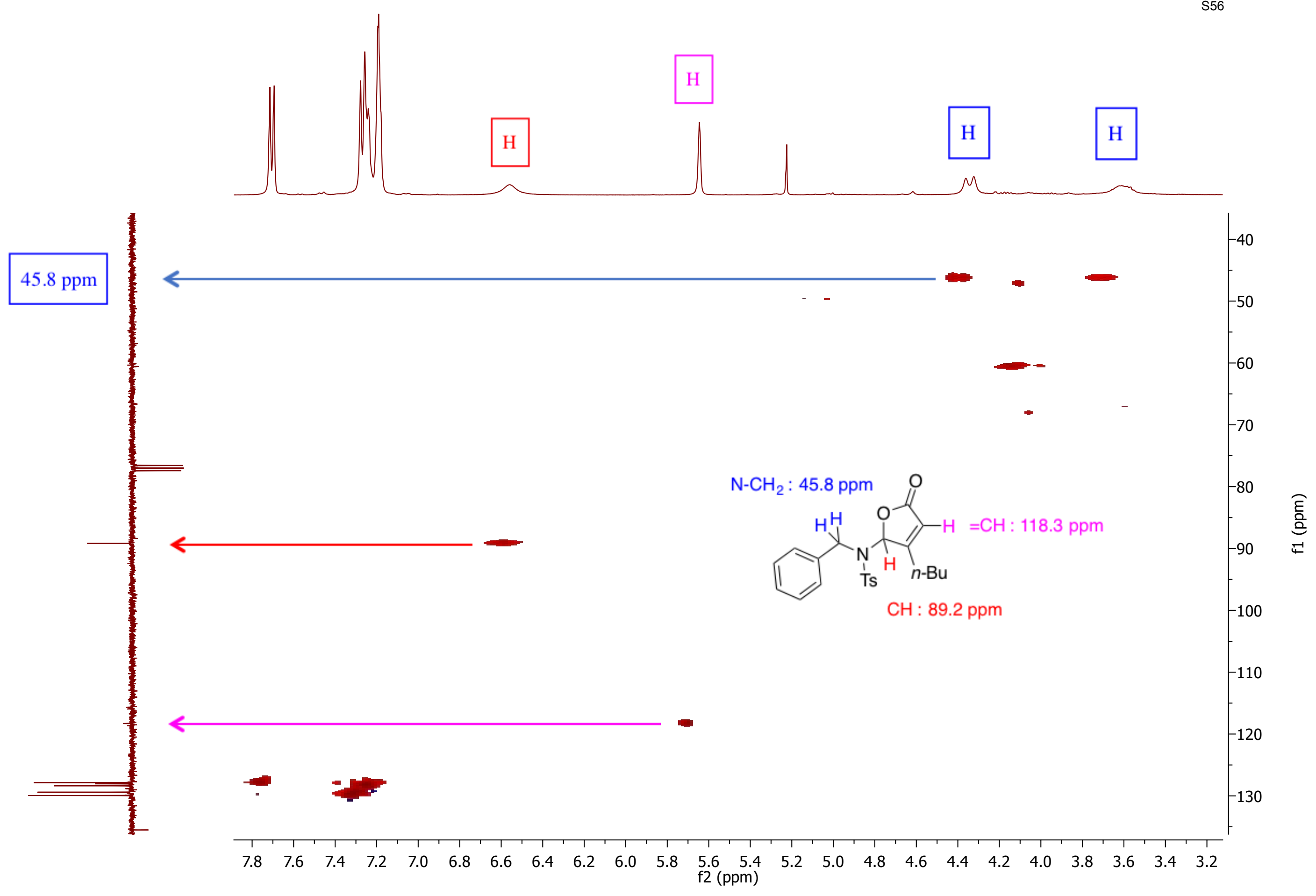



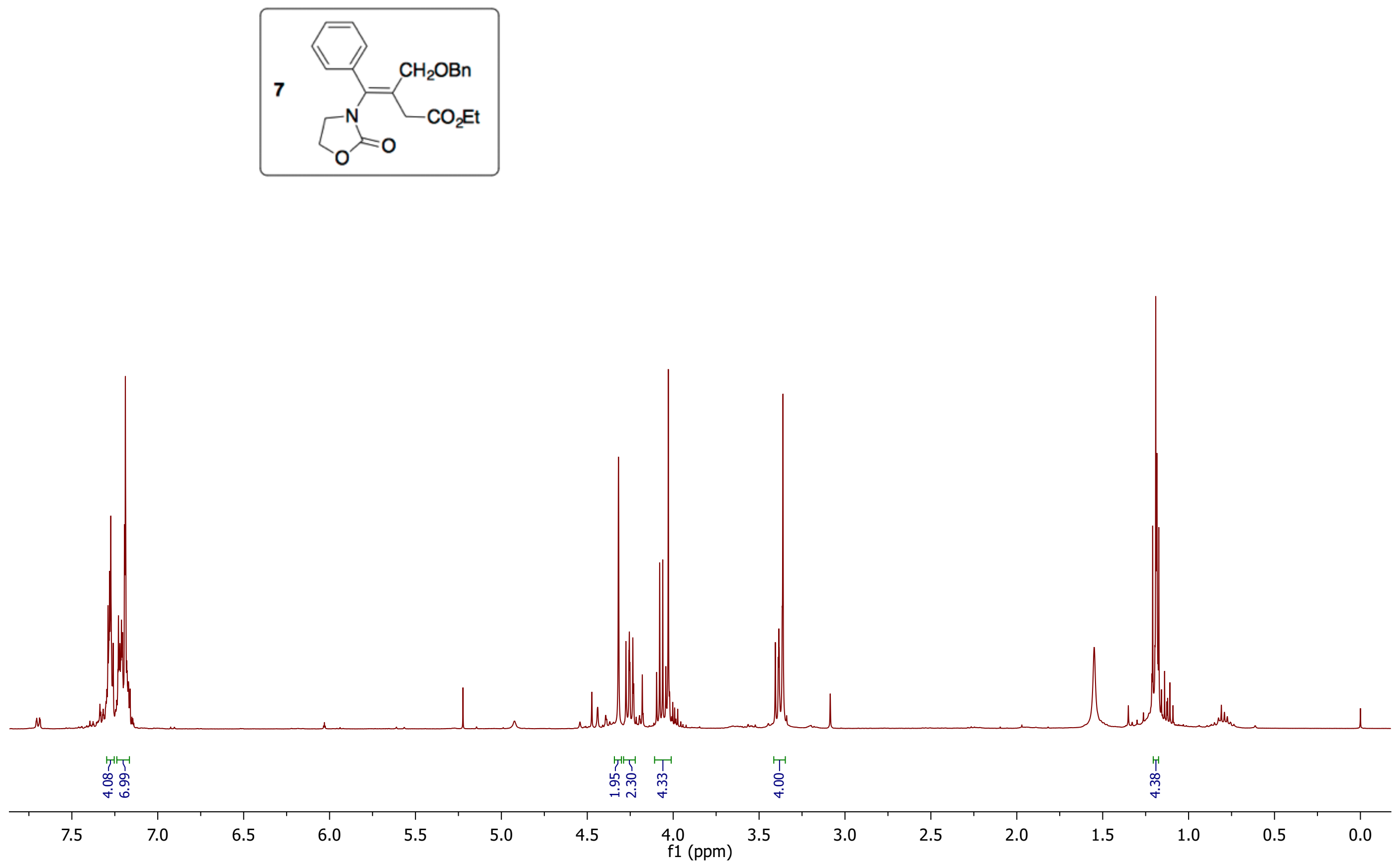


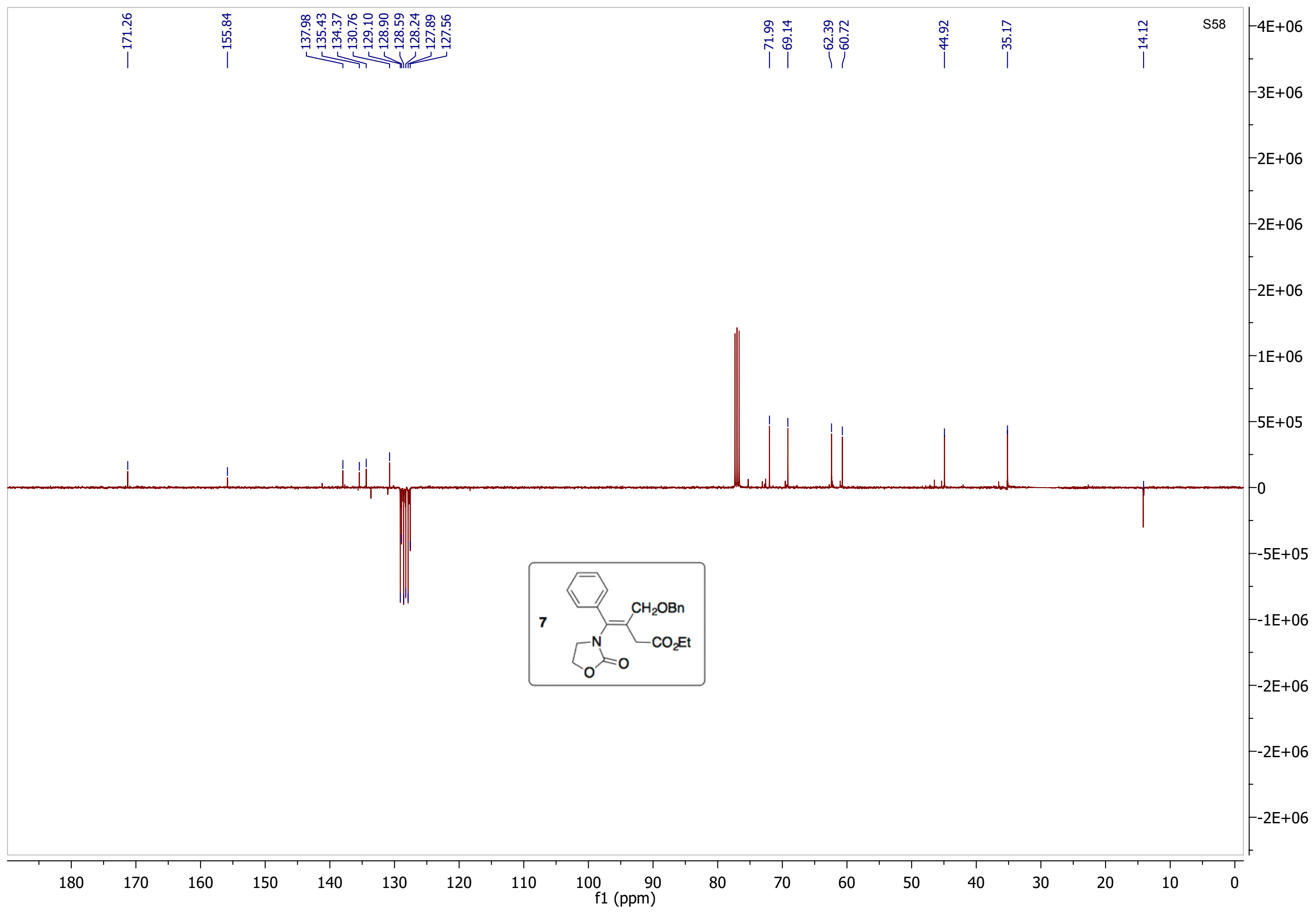




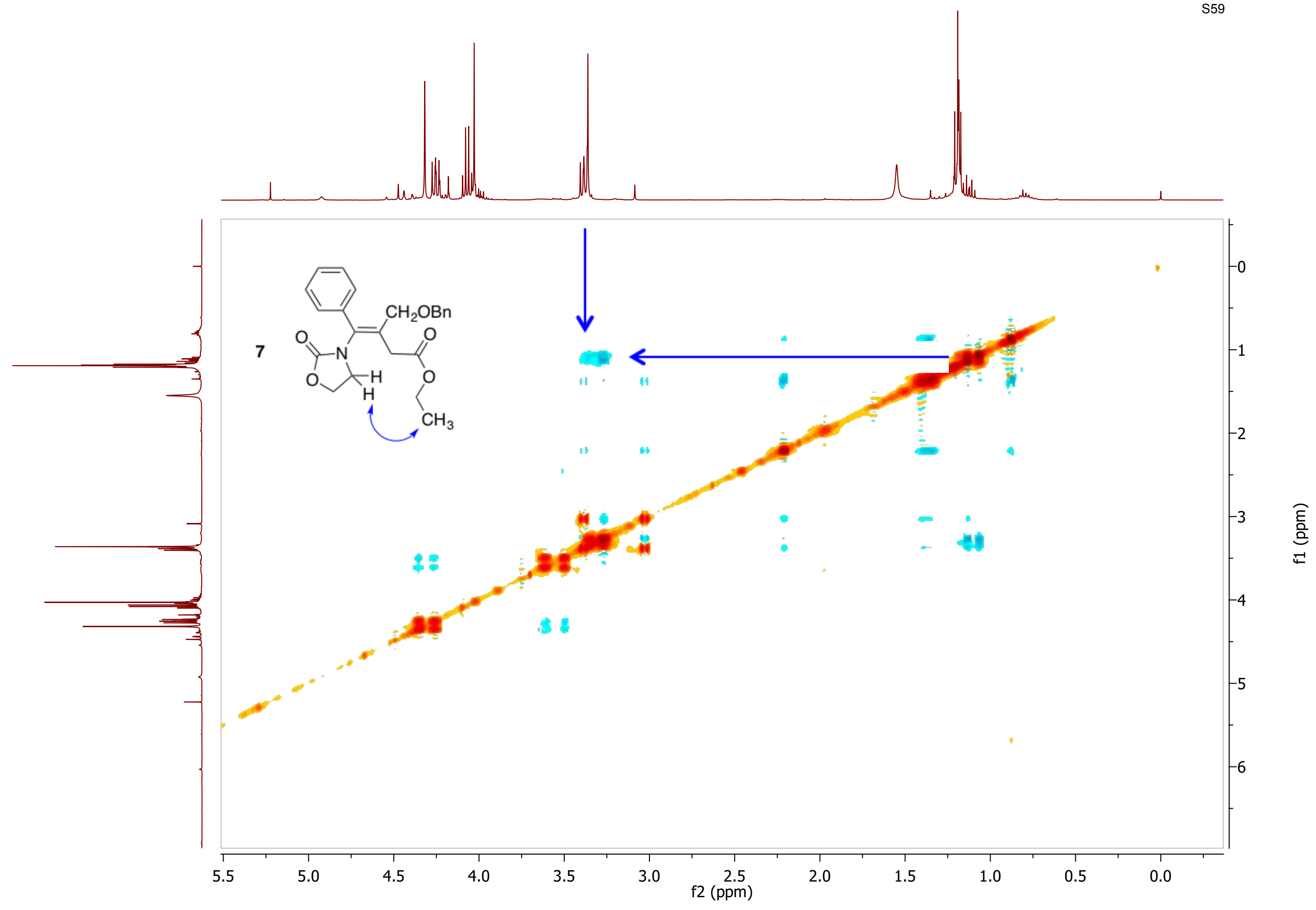

